\title{
2008
}

\section{Savannah River Site Annual Illness and Injury Surveillance Report}

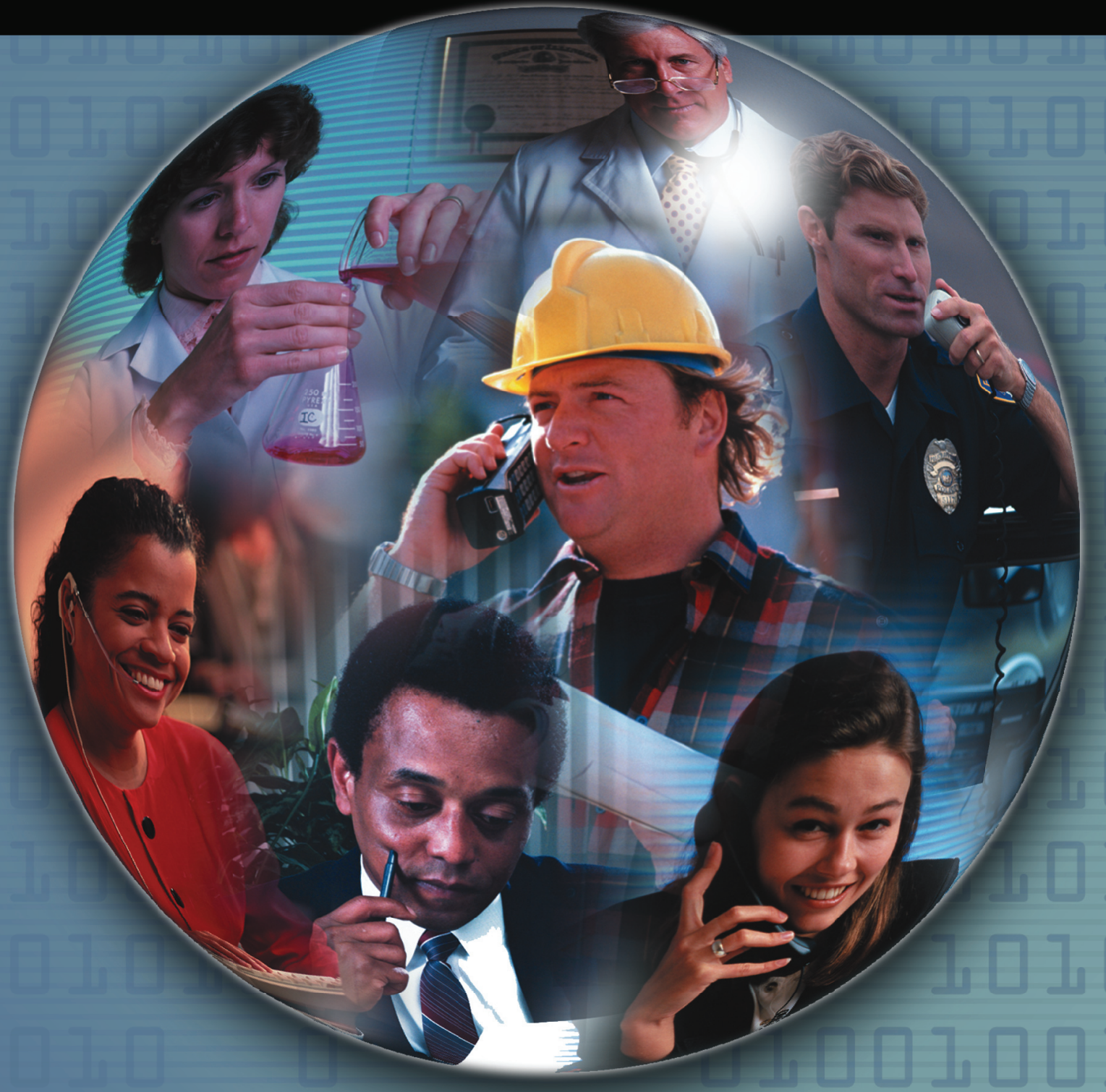




\section{Savannah River Site 2008 Illness and Injury Surveillance Report}

Questions or comments about this report or the Illness and Injury Surveillance Program (IISP) may be directed to:

E-mail:

Dr. Cliff Strader at cliff.strader@hq.doe.gov or Dr. Bonnie Richter at bonnie.richter@hq.doe.gov

or direct letters to:

Mail Stop HS-13 / 270CC

U.S. Department of Energy

1000 Independence Avenue, S.W.

Washington, DC 20585-0270

Additional information about the Department of Energy's Office of Illness and Injury Prevention Programs, the IISP, and annual reports for DOE sites participating in this program can be found at:

http://www.hss.energy.gov/healthsafety/WSHP/epi/surv/

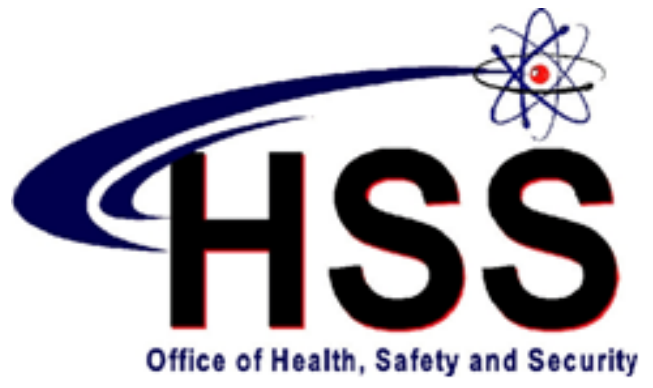

ACKNOWLEDGEMENT

LifeART images copyright 2000 Lippincott Williams \& Wilkins. All rights reserved.

This document was produced under contract number DE-AC05-06OR23100 between the

U.S. Department of Energy and Oak Ridge Associated Universities. 


\section{Savannah River Site 2008 Illness and Injury Surveillance Report}

\section{At A Glance}

The 9,569 employees at Savannah River Site (SRS) in 2008 represented an increase of 7 percent from 2007. The SRS work force has declined 21 percent since 2003, when there were 12,111 workers. Since 2003, women have accounted for approximately 25 percent of the work force. The average age of Savannah River Site workers has remained constant since 2005, with an average age of 49 years and 47 years among men and women, respectively.

Savannah River Site reported 3,808 absences in 2008, a 14 percent increase from the 3,347 absences reported in 2007. The increase in absences is not attributed to any particular diagnostic category. The absence rate has increased steadily since 2006. The explanation for this increase is unclear.

In 2008, the work force absence rate was 58.2 per 100 women and 34.0 per 100 men. The higher absence rate among women than among men is consistent with SRS absence rates since 2003. The distribution of diagnoses has been similar for men and women over this time period. In general, women reflect higher absence rates than do men across the sites participating in the Illness and Injury Surveillance Program (IISP). The average length of absence among SRS workers was 16 days for women and men in 2008.

Women in the work force lost 22,074 calendar days due to illness and injury in 2008.

Respiratory conditions (24 percent), musculoskeletal conditions (19 percent), and unspecified symptoms (11 percent) accounted for 54 percent of all reported diagnoses.

Men lost 39,840 calendar days due to illness and injury. Over half of their reported diagnoses (52 percent) involved respiratory conditions (24 percent), musculoskeletal conditions (17 percent), and unspecified symptoms (11 percent).

Respiratory disorders, unspecified symptoms, and disorders of the musculoskeletal system were the top 3 diagnosis categories reported by men and women at SRS from 2003 to 2008. Diagnosis rates for disorders of the respiratory and musculoskeletal systems were among the highest observed for all DOE IISP sites from 1998 to 2007. 
Line Operators had the highest absence rate and Crafts workers had the lowest absence rate among both women and men. The distribution of diagnoses is similar for each occupational group. Women in the Security and Fire occupational category reported no absences in 2008. The high rate among Line Operators was typical of the pattern noted among all IISP sites as a group from 1998 through 2007.

In 2008, SRS workers reported 62 sentinel health event diagnoses. Carpal tunnel syndrome accounted for 19 of these diagnoses. Workers reporting carpal tunnel syndrome were aged 40 and above and most frequently employed in the Technical Support occupational group. There were 5 diagnoses of chronic beryllium disease. The remaining SHEO diagnoses were not attributed to any particular diagnostic category.

In 2008, SRS reported 33 OSHA events, a 13 percent increase over the 29 OSHA events reported in 2007. The 2007 total was the lowest number of OSHA events reported since 2003. The number of lost or restricted workdays reported in 2008 decreased to 648 days compared with 842 days in 2007.

Eleven (33 percent) of the 33 workers reporting an OSHA event were Line Operators. This occupational group comprised only 18 percent of the work force. Falls accounted for 3 of the 11 events; 1 fall resulted in 142 restricted workdays. 
The Savannah River Site

\section{Work Force - 2008}

The Work Force by Gender and Age .. 1

The Work Force by Gender and Job

Category 1

\section{Number and Length of Absences}

Absence Rate by Gender and Age 2

Number of Days Absent by

Gender and Age

Absence Rate by Job Category

and Gender .. 3

Average Duration of Absence by

Job Category and Gender. .. 3

\section{Diagnostic Categories}

Number of Diagnoses and Lost Calendar Days by Diagnostic Category

(Categorized by ICD-9-CM) and Gender

Common Diagnoses Among Female

Workers in 2008 . 5

Common Diagnoses Among Male

Workers in 2008 .... 6

Number of Most Frequently Reported Diagnoses by Job Category and Gender 7

\section{Rates of Disease Occurrence}

Rates for All Illnesses and Injuries Combined by Job Category, Gender, and Age

Rates for Selected Diagnostic Categories by Job Category, Gender, and Age . .8

\section{Time Trends}

Age-Adjusted Rates for All Diagnoses Combined Among Women and Men from 2003 to 2008
Age-Adjusted Rates for Selected Diagnostic Categories Among Women and Men from 2003 to 2008

Age-Adjusted Rates for All Diagnoses

Combined Among Women and Men by Job

Category from 2003 to $2008 \ldots \ldots \ldots \ldots \ldots \ldots \ldots \ldots . \ldots 12$

\section{Sentinel Health Events for Occupations (SHEOs)}

Characteristics of SHEOs by Gender 13

SHEO Diagnoses by Gender 13

\section{Occupational Safety and Health Administration (OSHA)-Recordable Events}

OSHA-Recordable Events by Gender and Age. 14

OSHA-Recordable Events by Job

Category and Gender 14

\section{Diagnostic and Accident Categories for OSHA-Recordable Events}

OSHA-Recordable Diagnoses by

Diagnostic Category and Gender

OSHA-Recordable Accidents by Type

and Gender....

\section{Rates of OSHA-Recordable Events}

OSHA-Recordable Rates by Age and Job

Categories Among Women, All Diagnoses

Combined

OSHA-Recordable Rates by Age and Job

Categories Among Men, All Diagnoses

Combined

Time Trends for OSHA-Recordable Events

Age-Adjusted Rates for All OSHA-Recordable Diagnoses Combined Among Women and Men by Job Category from 2003 to 2008 .

\section{Appendices}

Appendices A-W 19 
The Savannah River Site Work Force - 2008

Figure 1. The Work Force by Gender and Age

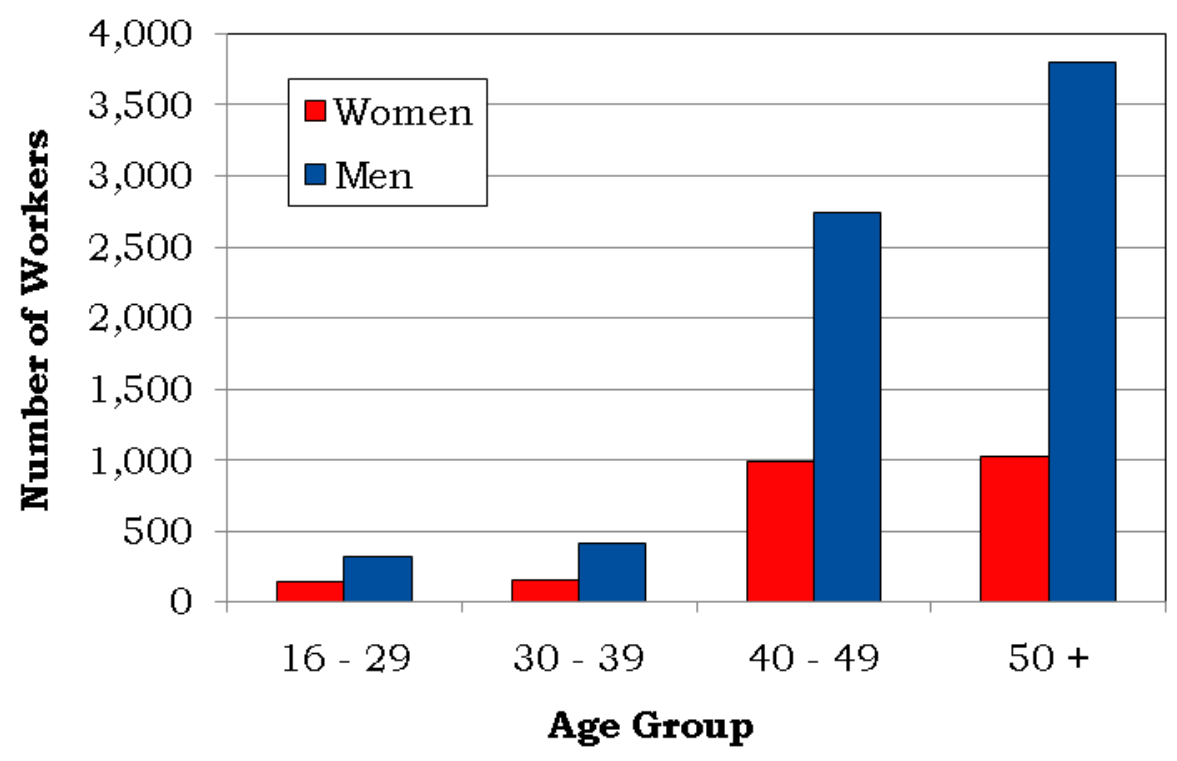

Figure 2. The Work Force by Gender and Job Category

\begin{tabular}{|l|c|c|}
\hline \multicolumn{1}{|c|}{ Job Category } & Women & M en \\
\hline Professional & 609 & 2,167 \\
& $26 \%$ & $30 \%$ \\
\hline \multirow{2}{*}{ Administrative Support } & 608 & 303 \\
& $26 \%$ & $4 \%$ \\
\hline Technical Support & 498 & 1,355 \\
& $22 \%$ & $19 \%$ \\
\hline \multirow{2}{*}{ Service } & 173 & 1,268 \\
& $\mathbf{8} \%$ & $17 \%$ \\
\hline \multirow{2}{*}{ Security and Fire } & 3 & 31 \\
& $<1 \%$ & $<1 \%$ \\
\hline \multirow{2}{*}{ Crafts } & 121 & $\mathbf{7 1 7}$ \\
& $\mathbf{5} \%$ & $10 \%$ \\
\hline \multirow{2}{*}{ Line Operators } & 292 & 1,424 \\
& $13 \%$ & $20 \%$ \\
\hline \multirow{2}{*}{ Total } & 2,304 & $\mathbf{7 , 2 6 5}$ \\
& $100 \%$ & $100 \%$ \\
\hline
\end{tabular}




\section{Number and Length of Absences}

Figure 3. Absence Rate by Gender and Age

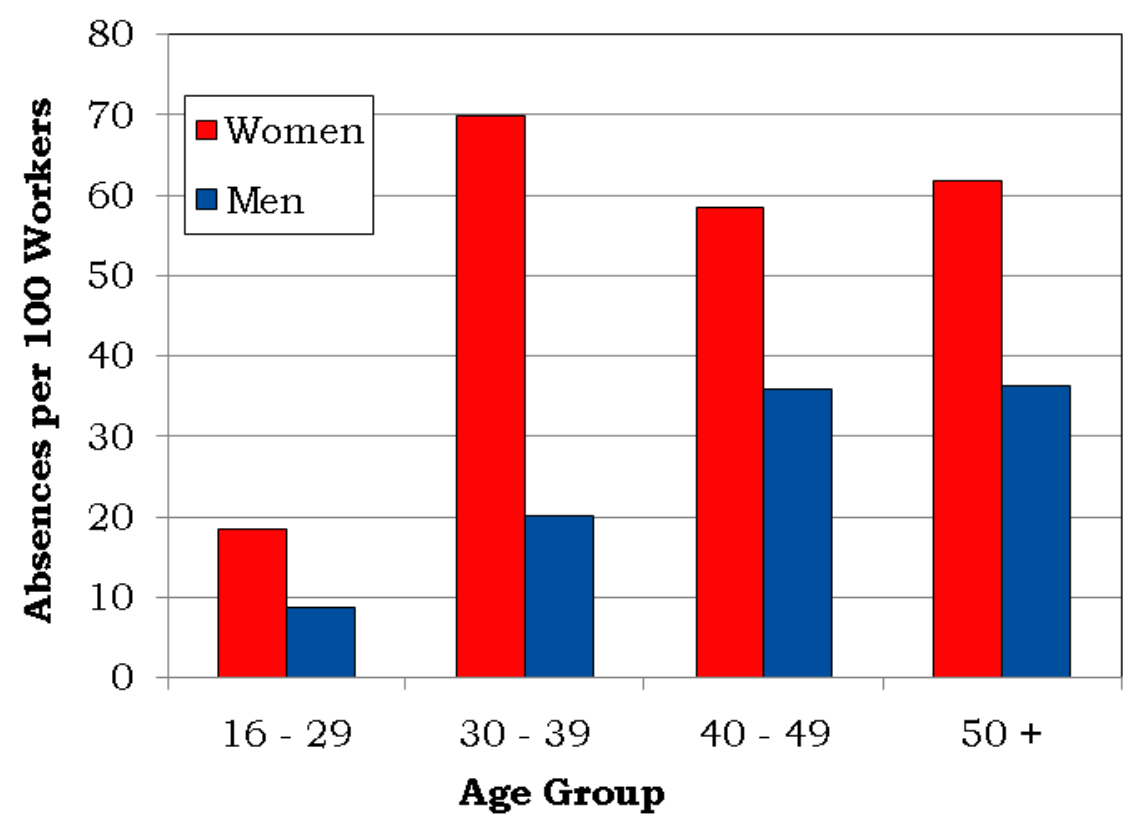

Figure 4. Number of Days Absent by Gender and Age

\begin{tabular}{|c|c|c|c|c|}
\hline \multirow{2}{*}{ Gender } & \multirow{2}{*}{ Age } & $\begin{array}{c}\text { Number of } \\
\text { Absences }\end{array}$ & \multicolumn{2}{|c|}{ Number of Days Absent } \\
\cline { 3 - 5 } & & Total & Average \\
\hline \multirow{4}{*}{ Women } & $16-29$ & 26 & 235 & 9 \\
\cline { 2 - 5 } & $30-39$ & 102 & 1,327 & 13 \\
\cline { 2 - 5 } & $40-49$ & 579 & 9,969 & 17 \\
\cline { 2 - 5 } & $50+$ & 634 & 10,543 & 17 \\
\cline { 2 - 5 } & Total & 1,341 & 22,074 & 16 \\
\hline \multirow{4}{*}{ Men } & $16-29$ & 27 & 399 & 15 \\
\cline { 2 - 5 } & $30-39$ & 85 & 1,386 & 16 \\
\cline { 2 - 5 } & $40-49$ & 983 & 13,985 & 14 \\
\cline { 2 - 5 } & $50+$ & 1,372 & 24,070 & 18 \\
\cline { 2 - 5 } & Total & 2,467 & 39,840 & 16 \\
\hline
\end{tabular}


Figure 5. Absence Rate by Job Category and Gender

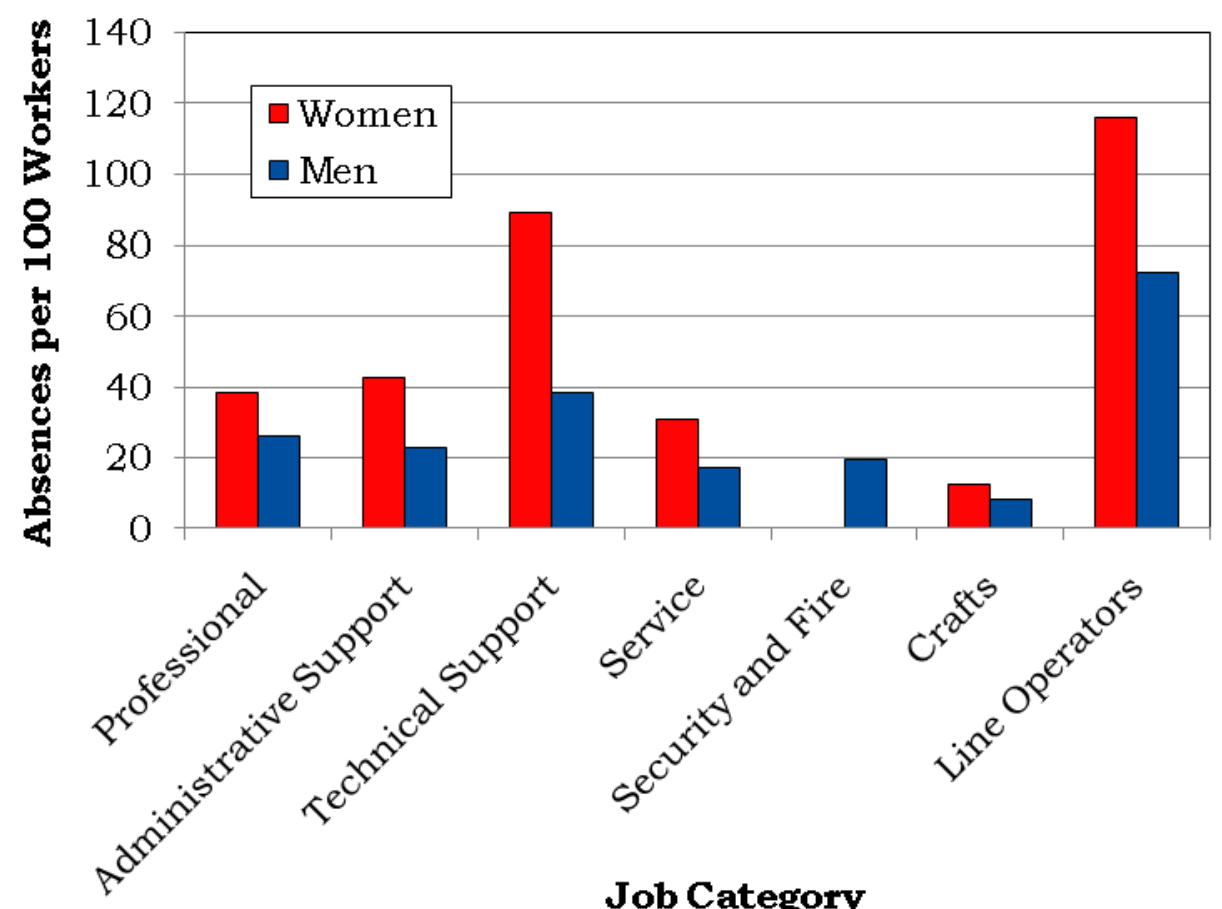

Figure 6. Average Duration of Absence by Job Category and Gender

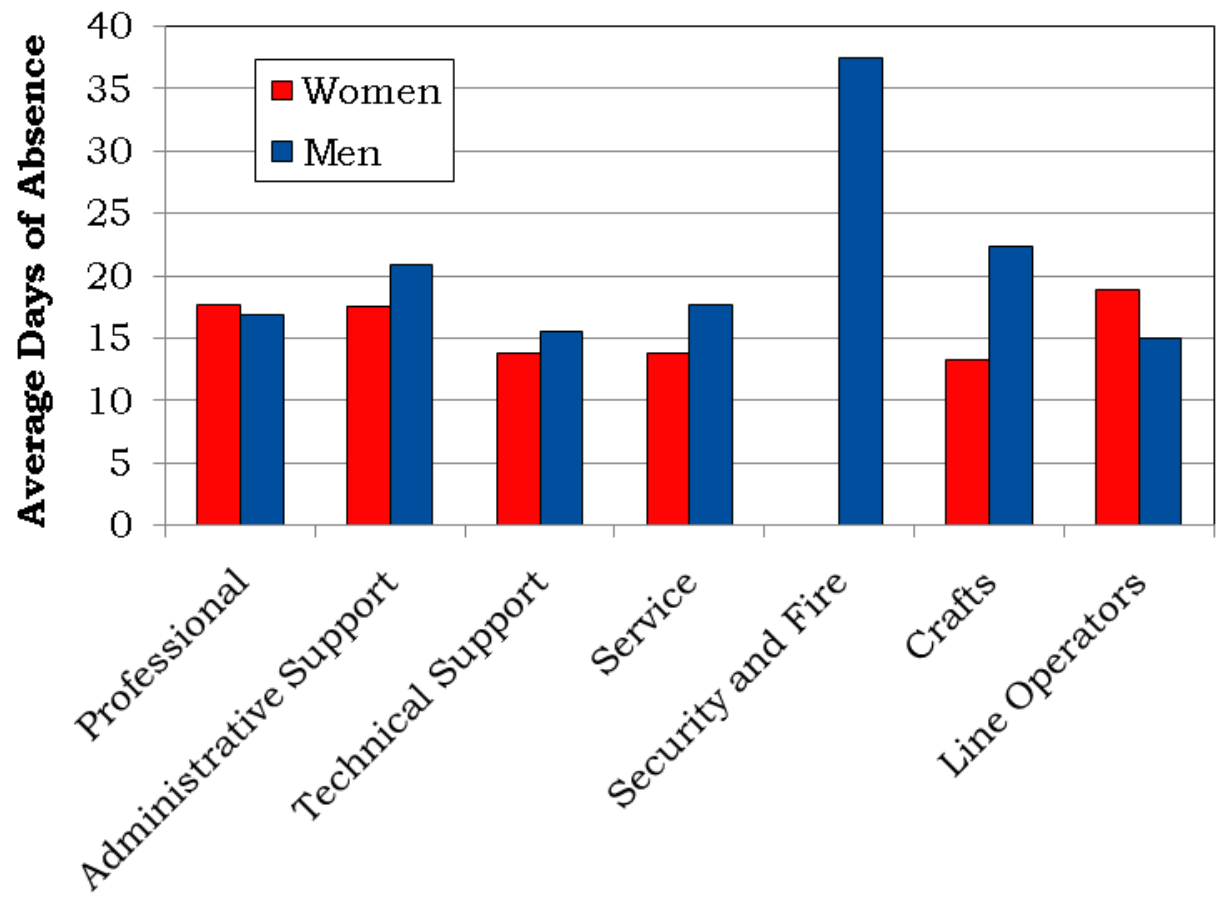

Job Category 


\section{Diagnostic Categories}

Figure 7. Number of Diagnoses and Lost Calendar Days by Diagnostic Category (Categorized by ICD-9-CM) and Gender

\begin{tabular}{|c|c|c|c|c|}
\hline \multirow[b]{2}{*}{ Diagnostic Category } & \multicolumn{2}{|c|}{ Women } & \multicolumn{2}{|c|}{ Men } \\
\hline & $\begin{array}{l}\text { Number of } \\
\text { Diagnoses }\end{array}$ & $\begin{array}{c}\text { Number } \\
\text { of Lost } \\
\text { Calendar } \\
\text { Days }\end{array}$ & $\begin{array}{l}\text { Number of } \\
\text { Diagnoses }\end{array}$ & $\begin{array}{c}\text { Number } \\
\text { of Lost } \\
\text { Calendar } \\
\text { Days }\end{array}$ \\
\hline Benign Growths & 34 & 1,019 & 30 & 553 \\
\hline Blood & 29 & 615 & 8 & 164 \\
\hline Cancer & 31 & 783 & 97 & 3,042 \\
\hline Digestive & 153 & 2,575 & 332 & 5,062 \\
\hline Endocrine / Me tabolic & 50 & 1,013 & 128 & 2,605 \\
\hline Existing Birth Condition & $\mathbf{0}$ & $\mathbf{0}$ & 1 & 4 \\
\hline Genitourinary & 199 & 3,401 & 187 & 2,284 \\
\hline Heart/Circulatory & 108 & 1,645 & 277 & 5,091 \\
\hline Infections/Parasites & 70 & 662 & 145 & 1,893 \\
\hline Injury & 149 & 2,413 & 352 & 6,750 \\
\hline Miscarriage & 4 & 32 & NA & NA \\
\hline Musculoskeletal & 439 & 5,984 & 643 & 11,325 \\
\hline Nervous System & 138 & 1,830 & 206 & 3,165 \\
\hline Psychological & 32 & 807 & 49 & 1,236 \\
\hline Respiratory & 530 & 5,507 & 906 & 9,697 \\
\hline Skin & 23 & 322 & 43 & 598 \\
\hline Unspecified Symptoms & 242 & 2,603 & 440 & 4,991 \\
\hline
\end{tabular}

Note: Lost calendar days for each absence are counted more than once when multiple diagnoses occur in different diagnostic categories for the same absence. 
Figure 8. Common Diagnoses Among Female Workers in 2008

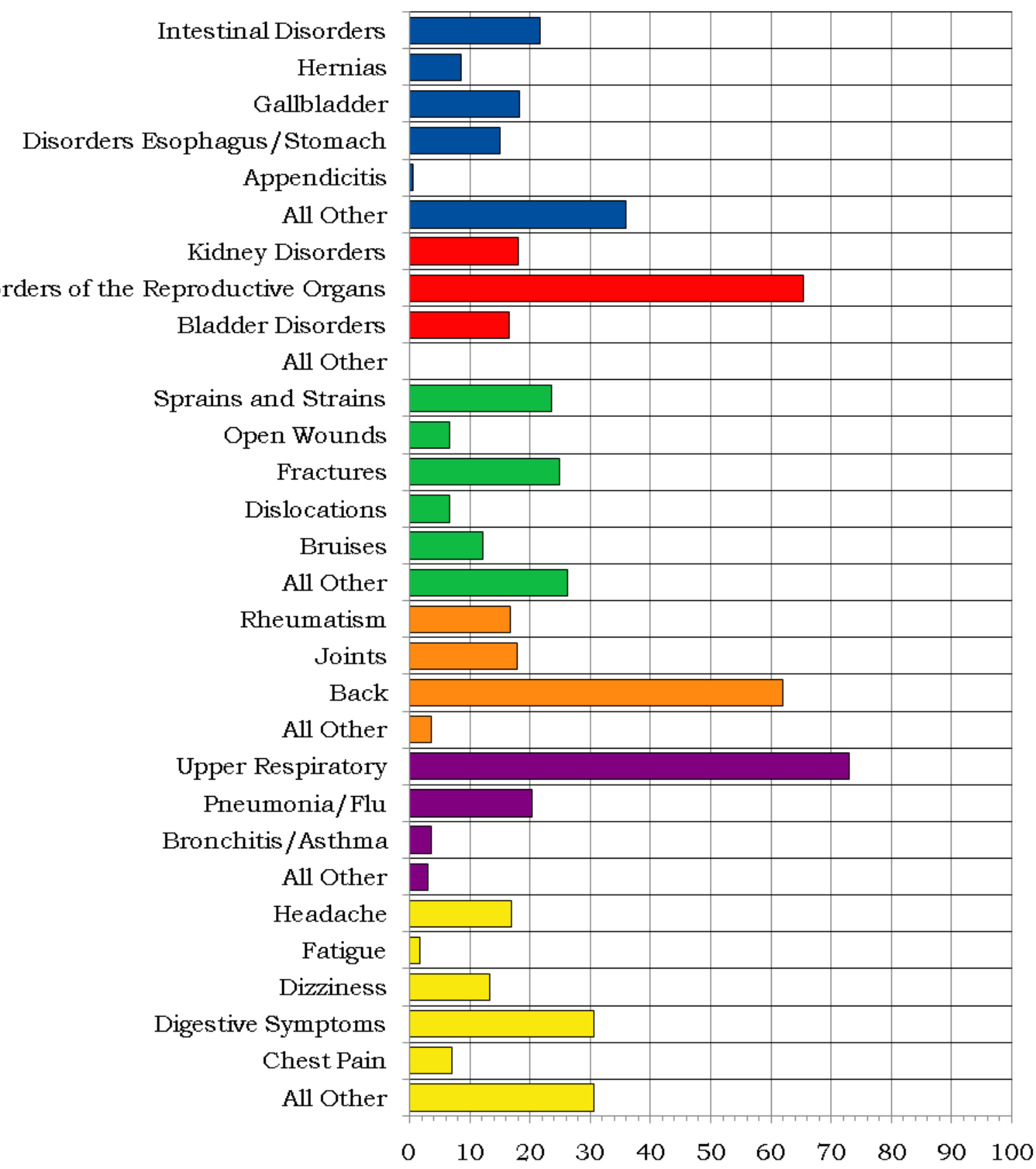

Percent Distribution of Diagnoses Within Diagnostic Category

Dige stive, 153 Diagnoses

Genitourinary, 199 Diagnoses

Injury, 149 Diagnoses
Musculoskeletal, 439 Diagnoses

Respiratory, 530 Diagnoses

Unspecified Symptoms, 242 Diagnoses 


\section{Figure 9. Common Diagnoses Among Male Workers in 2008}

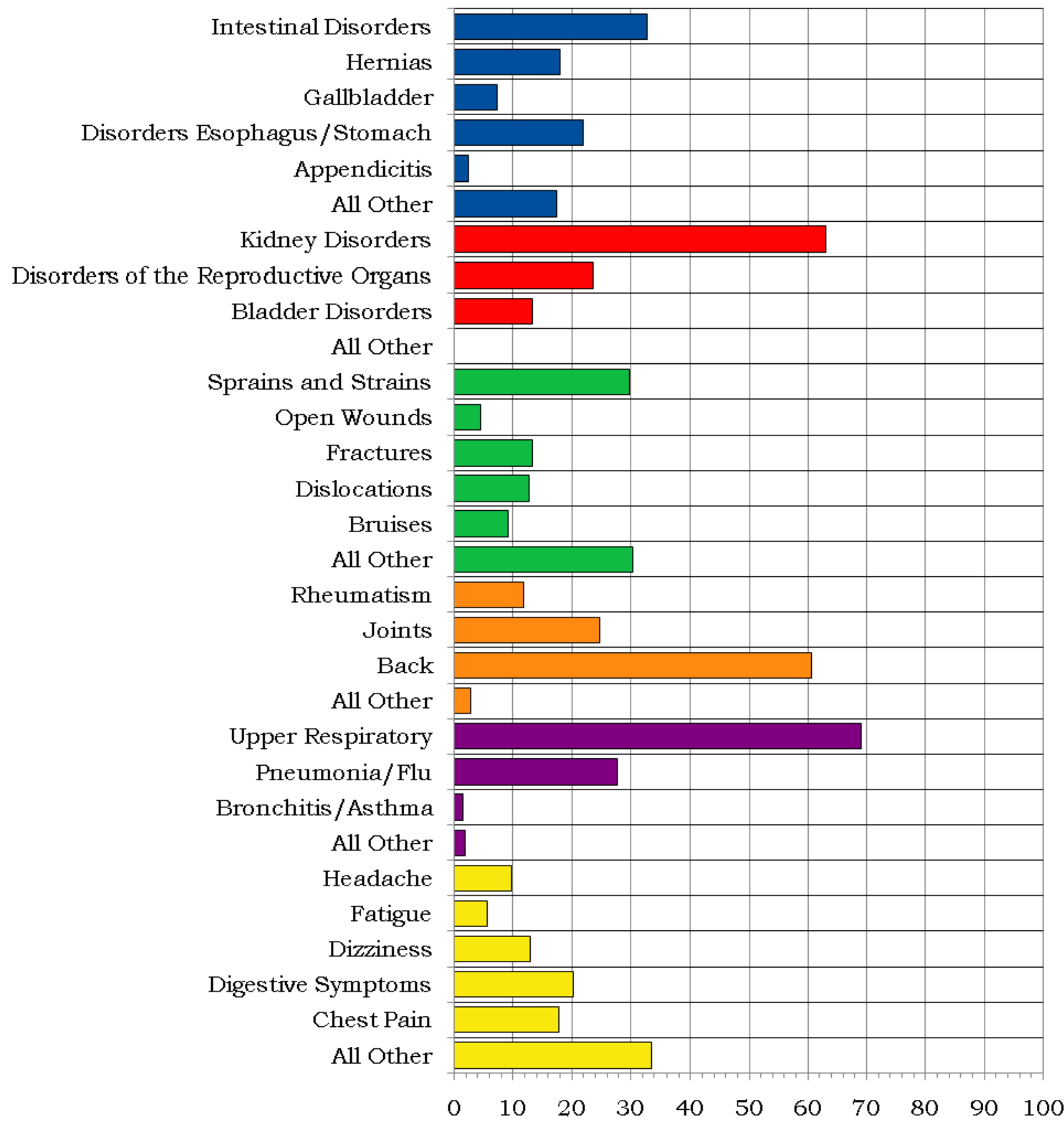

Percent Distribution of Diagnoses Within Diagnostic Category

Dige stive, 332 Diagnoses

Genitourinary, 187 Diagnoses

Injury, 352 Diagnoses
Musculoskeletal, 643 Diagnoses

Respiratory, 906 Diagnoses

Unspecified Symptoms, 440 Diagnoses 


\section{Figure 10. Number of Most Frequently Reported Diagnoses by Job Category and Gender}

\begin{tabular}{|c|c|c|c|c|}
\hline \multirow{4}{*}{\begin{tabular}{|l} 
Job Category \\
Professional
\end{tabular}} & \multicolumn{2}{|l|}{ Men } & \multicolumn{2}{|l|}{ Women } \\
\hline & Respiratory & 181 & M usculoskeletal & 103 \\
\hline & M usculoskeletal & 136 & Respiratory & 93 \\
\hline & Unspecified Symptoms & 124 & Genitourinary & 63 \\
\hline \multirow{3}{*}{ Administrative Support } & M usculoskeletal & 26 & Respiratory & 109 \\
\hline & Respiratory & 23 & M usculoskeletal & 88 \\
\hline & Digestive & 14 & Unspecified Symptoms & 54 \\
\hline \multirow{3}{*}{ Technical Support } & Respiratory & 202 & Respiratory & 170 \\
\hline & M usculoskeletal & 168 & M usculoskeletal & 129 \\
\hline & Injury & 78 & Unspecified Symptoms & 71 \\
\hline \multirow{3}{*}{ Service } & Respiratory & 49 & Respiratory & 18 \\
\hline & Injury & 49 & Digestive & 10 \\
\hline & M usculoskeletal & 47 & Nervous System & 10 \\
\hline \multirow{7}{*}{ Security and Fire } & Injury & 2 & & \multirow[t]{7}{*}{0} \\
\hline & Respiratory & 1 & & \\
\hline & Unspecified Symptoms & 1 & & \\
\hline & Digestive & 1 & & \\
\hline & Heart/Circulatory & 1 & & \\
\hline & Infections/Parasites & 1 & & \\
\hline & Benign Growths & 1 & & \\
\hline \multirow{3}{*}{ Crafts } & M usculoskeletal & 19 & Digestive & 7 \\
\hline & Respiratory & 9 & Unspecified Symptoms & 6 \\
\hline & Unspecified Symptoms & 9 & M usculoskeletal & 4 \\
\hline \multirow{3}{*}{ Line Operators } & Respiratory & 441 & Respiratory & 139 \\
\hline & M usculoskeletal & 247 & M usculoskeletal & 110 \\
\hline & Unspecified Symptoms & 197 & Unspecified Symptoms & 79 \\
\hline
\end{tabular}




\section{Rates of Disease Occurrence}

Figure 11. Rates for All Illnesses and Injuries Combined by Job Category, Gender, and Age

\begin{tabular}{|c|c|c|c|c|}
\hline \multirow{2}{*}{$\begin{array}{c}\text { All Illnesses \& } \\
\text { Injuries Combined }\end{array}$} & \multicolumn{4}{|c|}{ Rate per 1,000} \\
\hline & Job Category & Age & Men & Women \\
\hline & \multirow{2}{*}{ Professional } & $<50$ & 310 & 700 \\
\hline & & $50+$ & 535 & 695 \\
\hline & \multirow{2}{*}{ Administrative Support } & $<50$ & 296 & 560 \\
\hline & & $50+$ & 559 & 880 \\
\hline & \multirow{2}{*}{ Technical Support } & $<50$ & 521 & 1,549 \\
\hline & & $50+$ & 603 & 1,265 \\
\hline & \multirow{2}{*}{ Service } & $<50$ & 251 & 421 \\
\hline & & $50+$ & 275 & 250 \\
\hline & \multirow{2}{*}{ Security and Fire } & $<50$ & 304 & 0 \\
\hline & & $50+$ & 125 & 0 \\
\hline & \multirow{2}{*}{ Crafts } & $<50$ & 73 & 274 \\
\hline & & $50+$ & 149 & 54 \\
\hline & \multirow{2}{*}{ Line Operators } & $<<0$ & 1,128 & 1,783 \\
\hline & & $50+$ & 1,093 & 2,141 \\
\hline
\end{tabular}

Figure 12. Rates for Selected Diagnostic Categories by Job Category, Gender, and Age

\begin{tabular}{|c|c|c|c|c|}
\hline \multirow{2}{*}{ Cancer } & \multicolumn{4}{|c|}{ Rate per 1,000} \\
\hline & Job Category & Age & Men & Women \\
\hline & Drofecaionol & $<50$ & 10 & 38 \\
\hline & Frolessional & $50+$ & 17 & 0 \\
\hline & Administrative Sunport & $<50$ & 0 & 10 \\
\hline & 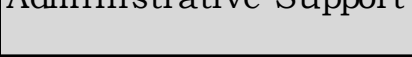 & $50+$ & 68 & 3 \\
\hline & Technical Support & $<50$ & 5 & 4 \\
\hline & 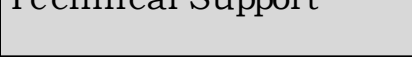 & $50+$ & 12 & 34 \\
\hline & Service & $<50$ & 3 & 0 \\
\hline & Net & $50+$ & 29 & 0 \\
\hline & & $<50$ & 0 & 0 \\
\hline & Security and Fire & $50+$ & $\mathbf{0}$ & 0 \\
\hline & Crafts & $<50$ & 0 & 0 \\
\hline & & $50+$ & 0 & 0 \\
\hline & Line Operators & $<50$ & 1 & 14 \\
\hline & & $50+$ & 31 & 20 \\
\hline
\end{tabular}


Figure 12. Rates for Selected Diagnostic Categories by Job Category, Gender, and Age (Continued)

\begin{tabular}{|l|l|c|c|c|}
\hline \multirow{2}{*}{ Heart/Circulatory } & \multicolumn{5}{|c|}{ Rate per 1,000 } \\
\cline { 2 - 5 } & \multicolumn{1}{|c|}{ Job Category } & Age & M en & W omen \\
\cline { 2 - 5 } & \multirow{2}{*}{ Professional } & $<50$ & $\mathbf{4 6}$ & $\mathbf{3}$ \\
\cline { 2 - 5 } & $50+$ & $\mathbf{4 8}$ & $\mathbf{3 4}$ \\
\cline { 2 - 5 } & \multirow{2}{*}{ Administrative Support } & $<50$ & $\mathbf{2 8}$ & $\mathbf{2 3}$ \\
\cline { 2 - 5 } & & $50+$ & $\mathbf{3 7}$ & $\mathbf{7 7}$ \\
\cline { 2 - 5 } & Technical Support & $<50$ & $\mathbf{1 8}$ & $\mathbf{5 7}$ \\
\cline { 2 - 5 } & $50+$ & $\mathbf{4 9}$ & $\mathbf{7 7}$ \\
\cline { 2 - 5 } & \multirow{2}{*}{ Service } & $<50$ & $\mathbf{1 2}$ & $\mathbf{0}$ \\
\cline { 2 - 5 } & $50+$ & $\mathbf{8}$ & $\mathbf{0}$ \\
\cline { 2 - 5 } & \multirow{2}{*}{ Security and Fire } & $<50$ & $\mathbf{0}$ & $\mathbf{0}$ \\
\cline { 2 - 5 } & $50+$ & $\mathbf{1 2 5}$ & $\mathbf{0}$ \\
\cline { 2 - 5 } & \multirow{2}{*}{ Crafts } & $<50$ & $\mathbf{1 1}$ & $\mathbf{0}$ \\
\cline { 2 - 5 } & $50+$ & $\mathbf{1 1}$ & $\mathbf{0}$ \\
\cline { 2 - 5 } & \multirow{2}{*}{ Line Operators } & $<50$ & $\mathbf{4 8}$ & $\mathbf{1 2 6}$ \\
\cline { 2 - 5 } & $50+$ & $\mathbf{8 4}$ & $\mathbf{1 1 4}$ \\
\hline
\end{tabular}

\begin{tabular}{|c|c|c|c|c|}
\hline \multirow{2}{*}{ Respiratory } & \multicolumn{4}{|c|}{ Rate per 1,000} \\
\hline & Job Category & Age & Men & Women \\
\hline & \multirow{2}{*}{ Professional } & $<50$ & 86 & 122 \\
\hline & & $50+$ & 81 & 192 \\
\hline & \multirow{2}{*}{ Administrative Support } & $<50$ & 92 & 149 \\
\hline & & $50+$ & 62 & 211 \\
\hline & \multirow{2}{*}{ Technical Support } & $<50$ & 135 & 318 \\
\hline & & $50+$ & 160 & 368 \\
\hline & \multirow{2}{*}{ Service } & $<50$ & 45 & 105 \\
\hline & & $50+$ & 32 & 100 \\
\hline & \multirow{2}{*}{ Security and Fire } & $<50$ & 43 & 0 \\
\hline & & $50+$ & 0 & 0 \\
\hline & \multirow{2}{*}{ Crafts } & $<50$ & 15 & 12 \\
\hline & & $50+$ & 8 & 0 \\
\hline & \multirow{2}{*}{ Line Operators } & $<50$ & 328 & 497 \\
\hline & & $50+$ & 293 & 456 \\
\hline
\end{tabular}


Figure 12. Rates for Selected Diagnostic Categories by Job Category, Gender, and Age (Continued)

\begin{tabular}{|c|c|c|c|c|}
\hline \multirow{2}{*}{ Injury } & \multicolumn{4}{|c|}{ Rate per 1,000} \\
\hline & Job Category & Age & Men & Women \\
\hline & \multirow{2}{*}{ Professional } & $<50$ & 10 & 26 \\
\hline & & $50+$ & 40 & 75 \\
\hline & \multirow{2}{*}{ Administrative Support } & $<50$ & 7 & 29 \\
\hline & & $50+$ & 37 & 33 \\
\hline & \multirow{2}{*}{ Technical Support } & $<50$ & 55 & 144 \\
\hline & & $50+$ & 60 & 85 \\
\hline & \multirow{2}{*}{ Service } & $<50$ & 26 & 30 \\
\hline & & $50+$ & 51 & 0 \\
\hline & \multirow{2}{*}{ Security and Fire } & $<50$ & 87 & 0 \\
\hline & & $50+$ & 0 & 0 \\
\hline & \multirow{2}{*}{ Crafts } & $<50$ & 13 & 24 \\
\hline & & $50+$ & 0 & 0 \\
\hline & \multirow{2}{*}{ Line Operators } & $<50$ & 121 & 70 \\
\hline & & $50+$ & 92 & 181 \\
\hline
\end{tabular}

\section{Time Trends}

Figure 13. Age-Adjusted Rates for All Diagnoses Combined Among Women and Men from 2003 to 2008*

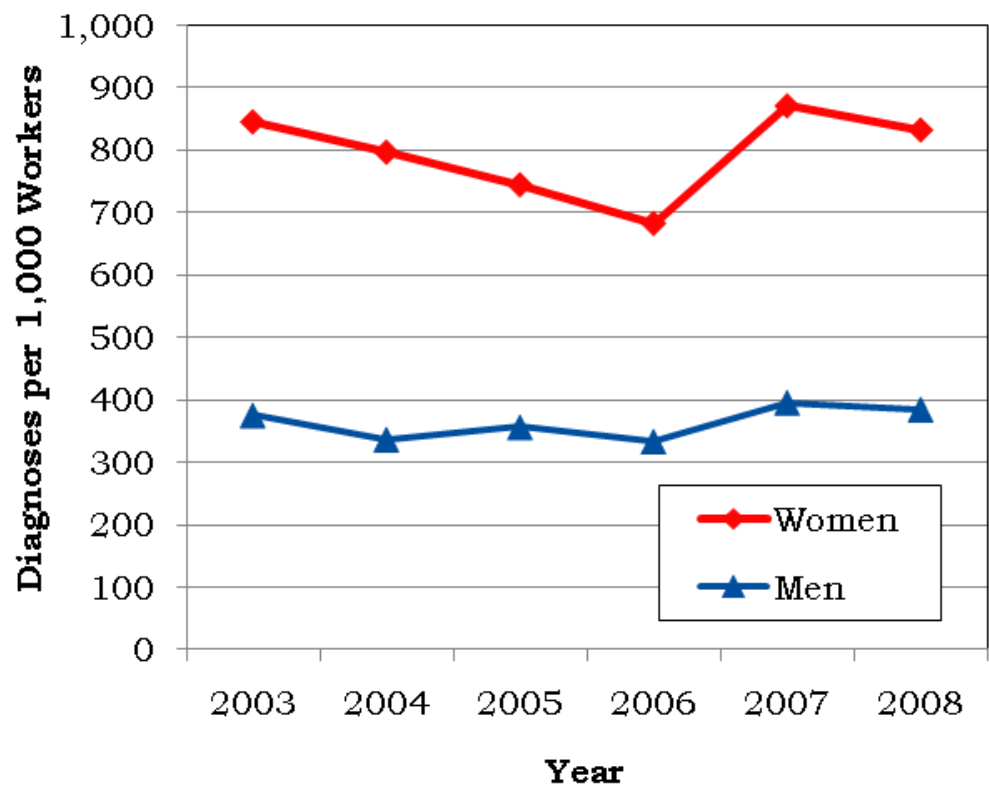

*Standardized to age distribution of 2000 U.S. population. 
Figure 14. Age-Adjusted Rates for Selected Diagnostic Categories Among Women and Men from 2003 to 2008*

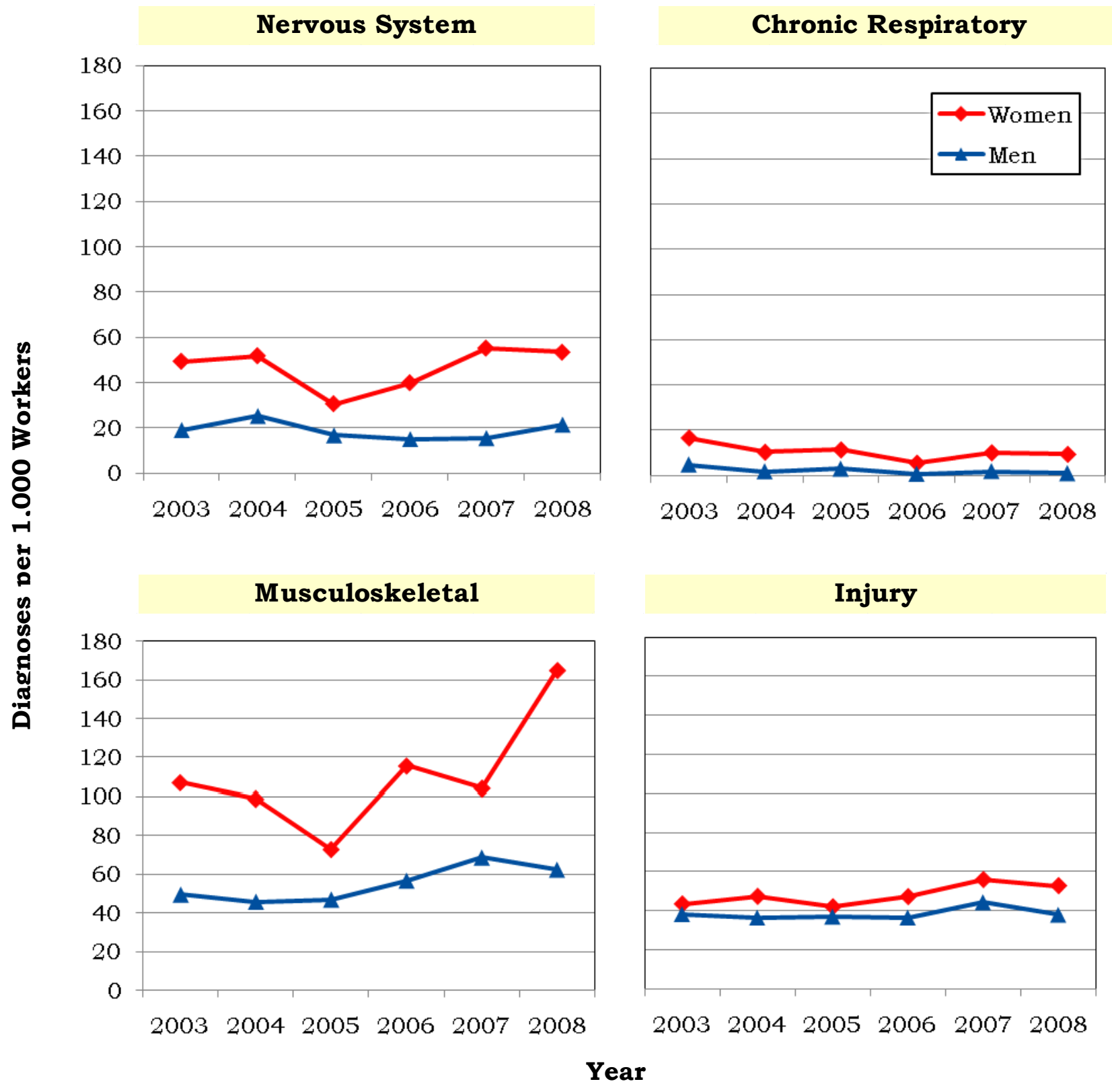

*Standardized to age distribution of 2000 U.S. population. 
Figure 15. Age-Adjusted Rates for All Diagnoses Combined Among Women and Men by Job Category from 2003 to 2008*

Professional

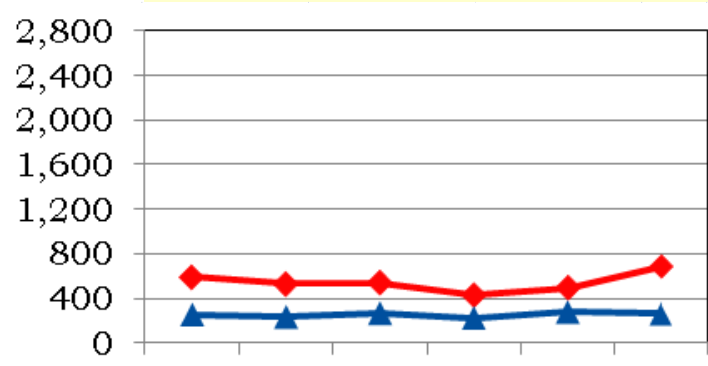

200320042005200620072008

Administrative Support

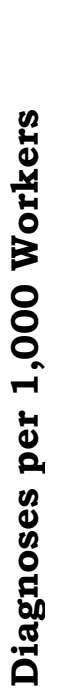

$$
2,800
$$$$
2,400
$$

2,000

1,600

1,200

800

400

0

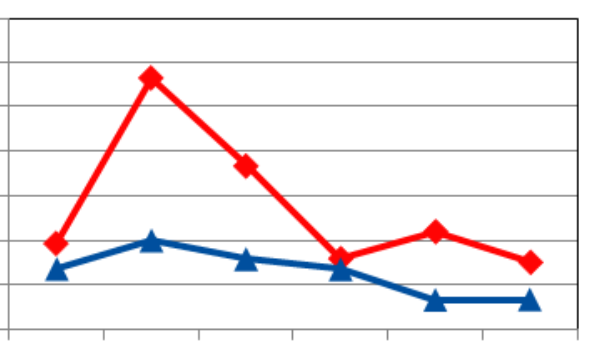

200320042005200620072008

Service

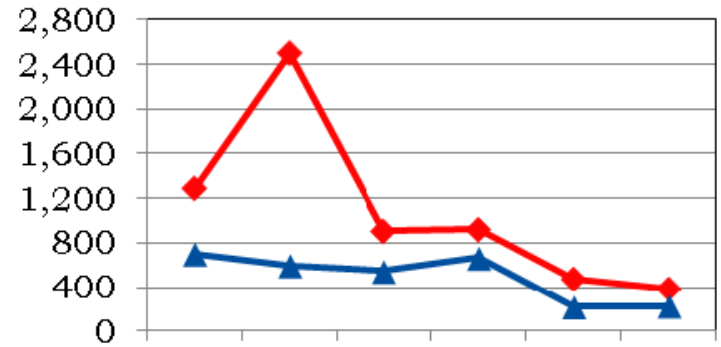

200320042005200620072008

\section{Crafts}

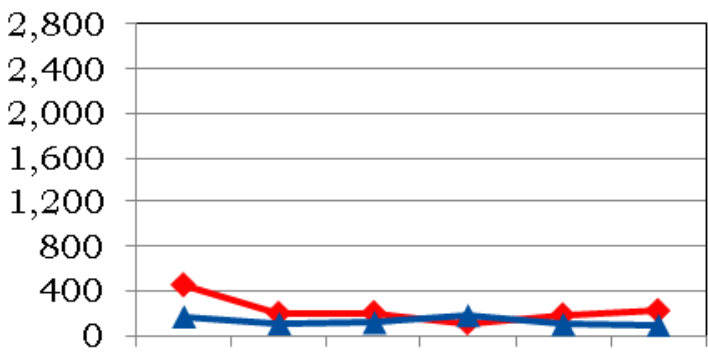

200320042005200620072008

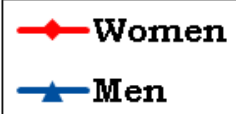

Technical Support

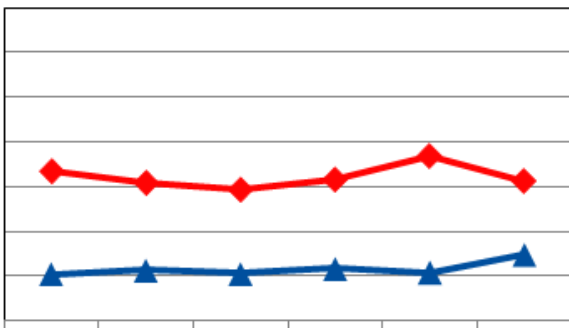

200320042005200620072008

Security and Fire

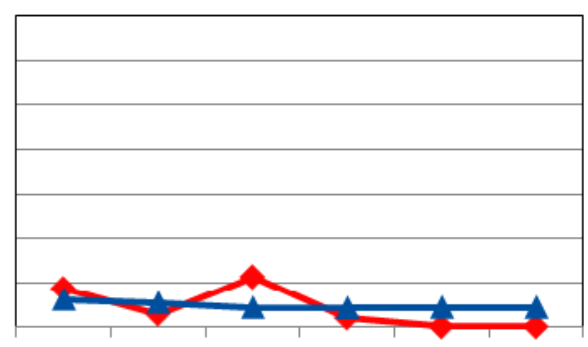

200320042005200620072008

Line Operators

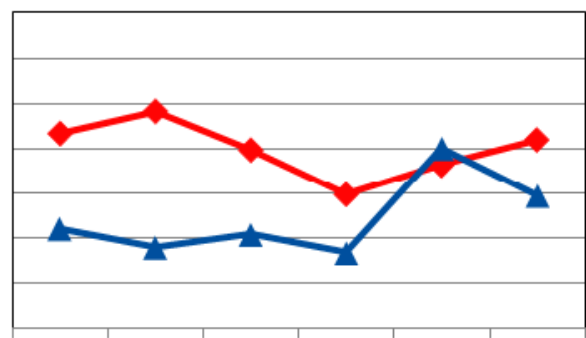

200320042005200620072008

Year

*Standardized to age distribution of 2000 U.S. population. 


\section{Sentinel Health Events for Occupations (SHEOs)}

An occupational sentinel health event (SHEO) is a disease, disability, or death that is likely to be occupationally related. Although sentinel health events may indicate an occupational exposure, many may result from nonoccupational exposures. Sentinel health events are therefore assessed in two categories:

Definite Sentinel Health Events: Diseases that are unlikely to occur in the absence of an occupational exposure (e.g., asbestosis).

Possible Sentinel Health Events: Diseases that may be occupational but can also occur in the absence of an occupational exposure (e.g., lung cancer or carpal tunnel syndrome).

Figure 16. Characteristics of SHEOs by Gender

\begin{tabular}{|l|c|c|c|c|}
\hline \multirow{2}{*}{} & \multicolumn{2}{|c|}{$\begin{array}{c}\text { Total Number of } \\
\text { SHEO Diagnoses }\end{array}$} & \multicolumn{2}{c|}{$\begin{array}{c}\text { Total Number of } \\
\text { Days Absent }\end{array}$} \\
\cline { 2 - 5 } & Men & Women & Men & Women \\
\hline Definite & 12 & 10 & 118 & 820 \\
\hline Possible & 30 & 10 & 465 & 1,117 \\
\hline Total & 42 & 20 & 583 & 1,937 \\
\hline
\end{tabular}

Figure 17. SHEO Diagnoses by Gender

\begin{tabular}{|l|c|c|}
\hline \multirow{2}{*}{ Diagnoses } & \multicolumn{2}{c|}{ Gender } \\
\cline { 2 - 3 } & Women & M en \\
\hline Carpal Tunnel Syndrome & 10 & 9 \\
\hline Pneumoconiosis (Berylliosis) & 0 & 5 \\
\hline M usculoskeletal Conditions & 1 & 1 \\
\hline Injuries & 5 & 1 \\
\hline Other Conditions & 4 & 26 \\
\hline
\end{tabular}


Occupational Safety and Health Administration (OSHA)-Recordable Events

Figure 18. OSHA-Recordable Events by Gender and Age

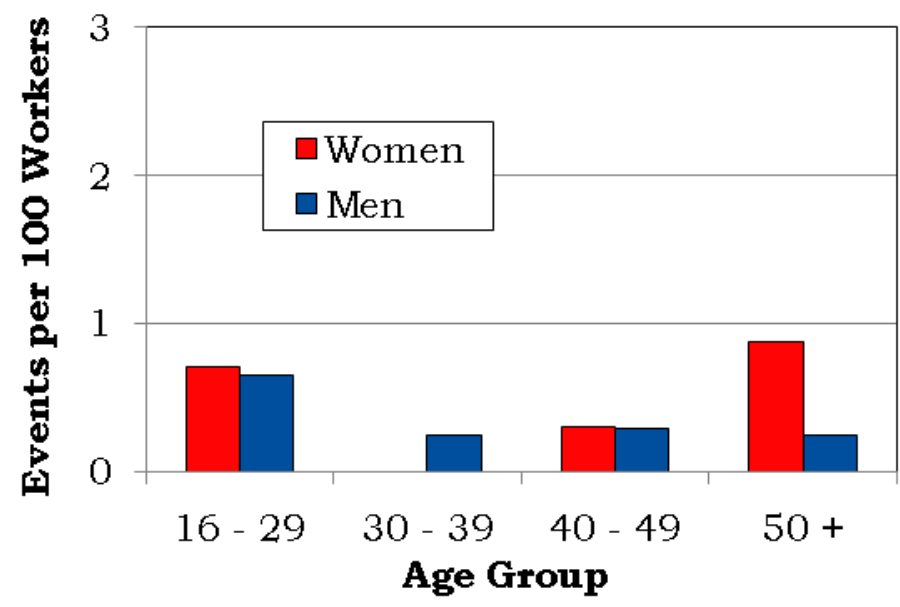

Figure 19. OSHA-Recordable Events by Job Category and Gender

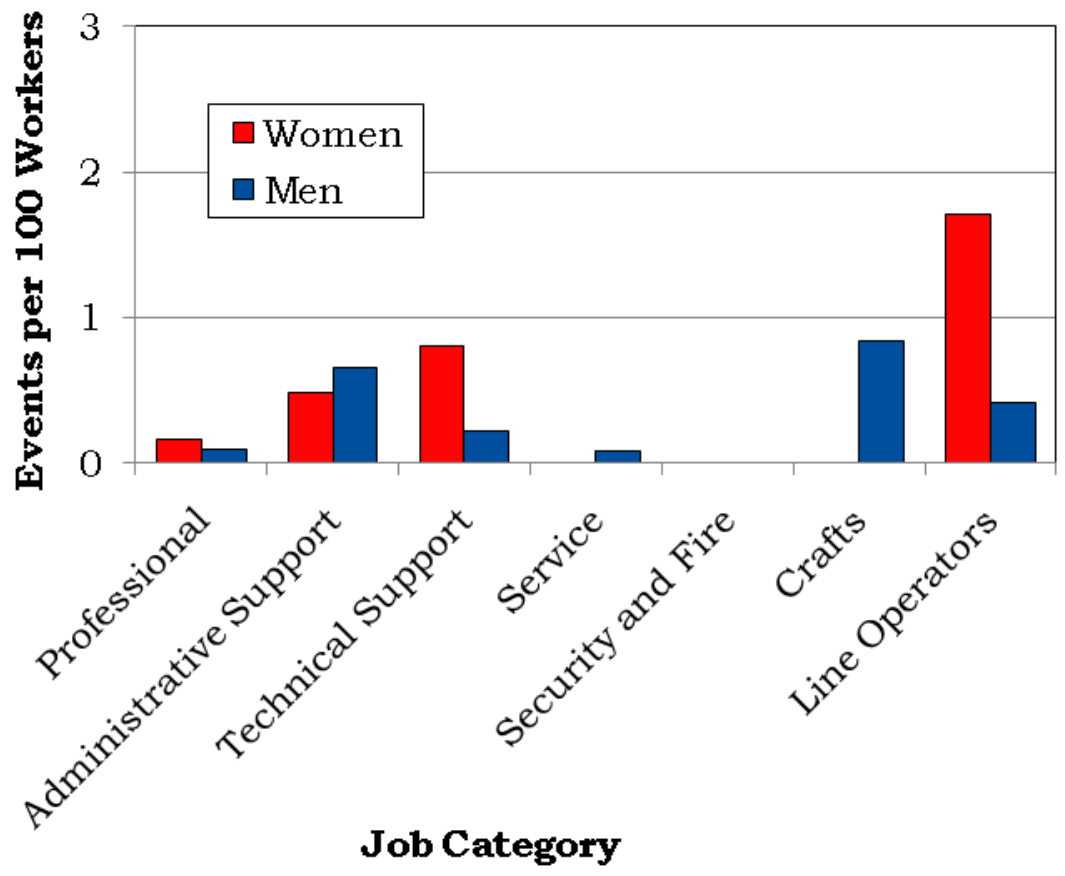




\section{Diagnostic and Accident Categories for OSHA-Recordable Events}

Figure 20. OSHA-Recordable Diagnoses by Diagnostic Category and Gender

\begin{tabular}{|l|c|c|}
\hline \multirow{2}{*}{ Diagnostic Category } & \multicolumn{2}{c|}{ Gender } \\
\cline { 2 - 3 } & Women & M en \\
\hline Endocrine / Metabolic & $\mathbf{1}$ & $\mathbf{0}$ \\
\hline Musculoskeletal & $\mathbf{4}$ & $\mathbf{5}$ \\
\hline Respiratory & $\mathbf{0}$ & $\mathbf{2}$ \\
\hline Unspecified Symptoms & $\mathbf{1 7}$ & $\mathbf{1}$ \\
\hline Injury & $\mathbf{1}$ & $\mathbf{1}$ \\
\hline Fractures - Upper Limb & $\mathbf{2}$ & $\mathbf{1}$ \\
\hline Fractures - Lower Limb & $\mathbf{0}$ & $\mathbf{1}$ \\
\hline Dislocations & $\mathbf{2}$ & $\mathbf{0}$ \\
\hline Back Sprains \& Strains & $\mathbf{1}$ & $\mathbf{5}$ \\
\hline Other Sprains \& Strains & $\mathbf{0}$ & $\mathbf{1}$ \\
\hline Open Wounds - Head, Neck, Trunk & $\mathbf{1}$ & $\mathbf{3}$ \\
\hline Open Wounds - Upper Limb & $\mathbf{1}$ & $\mathbf{2}$ \\
\hline Open Wounds - Lower Limb & $\mathbf{2}$ & $\mathbf{1}$ \\
\hline Superficial Injuries & $\mathbf{4}$ & $\mathbf{1}$ \\
\hline Bruises & $\mathbf{1}$ & $\mathbf{1}$ \\
\hline Foreign Bodies Entering Orifice & $\mathbf{1}$ & $\mathbf{0}$ \\
\hline Adverse Reactions to Non-Medical Substances & $\mathbf{1}$ & $\mathbf{0}$ \\
\hline Adverse Reactions to External Causes & & \\
\hline
\end{tabular}

Figure 21. OSHA-Recordable Accidents by Type and Gender

\begin{tabular}{|l|c|c|}
\hline \multirow{2}{*}{\multicolumn{1}{|c|}{ Accident Category }} & \multicolumn{2}{c|}{ Gender } \\
\cline { 2 - 3 } & Women & Men \\
\cline { 2 - 3 } & $\begin{array}{c}\text { Number of } \\
\text { Accidents }\end{array}$ & $\begin{array}{c}\text { Number of } \\
\text { Accidents }\end{array}$ \\
\hline Poi soning - Non-Medicinal & $\mathbf{1}$ & $\mathbf{0}$ \\
\hline Falls & $\mathbf{5}$ & $\mathbf{5}$ \\
\hline Natural/Environmental Factors & $\mathbf{1}$ & $\mathbf{0}$ \\
\hline Submersion/Suffocation/Foreign Bodies & $\mathbf{1}$ & $\mathbf{1}$ \\
\hline Other Accidents & $\mathbf{5}$ & $\mathbf{1 2}$ \\
\hline Struck by an Object & $\mathbf{1}$ & $\mathbf{5}$ \\
\hline Caught Between Objects & $\mathbf{1}$ & $\mathbf{0}$ \\
\hline Cutting/Piercing Instrument/Object & $\mathbf{1}$ & $\mathbf{1}$ \\
\hline Overexertion/Strenuous Movements & $\mathbf{2}$ & $\mathbf{6}$ \\
\hline Total & $\mathbf{1 3}$ & $\mathbf{1 8}$ \\
\hline
\end{tabular}




\section{Rates of OSHA-Recordable Events}

Figure 22. OSHA-Recordable Rates by Age and Job Categories Among Women, All Diagnoses Combined

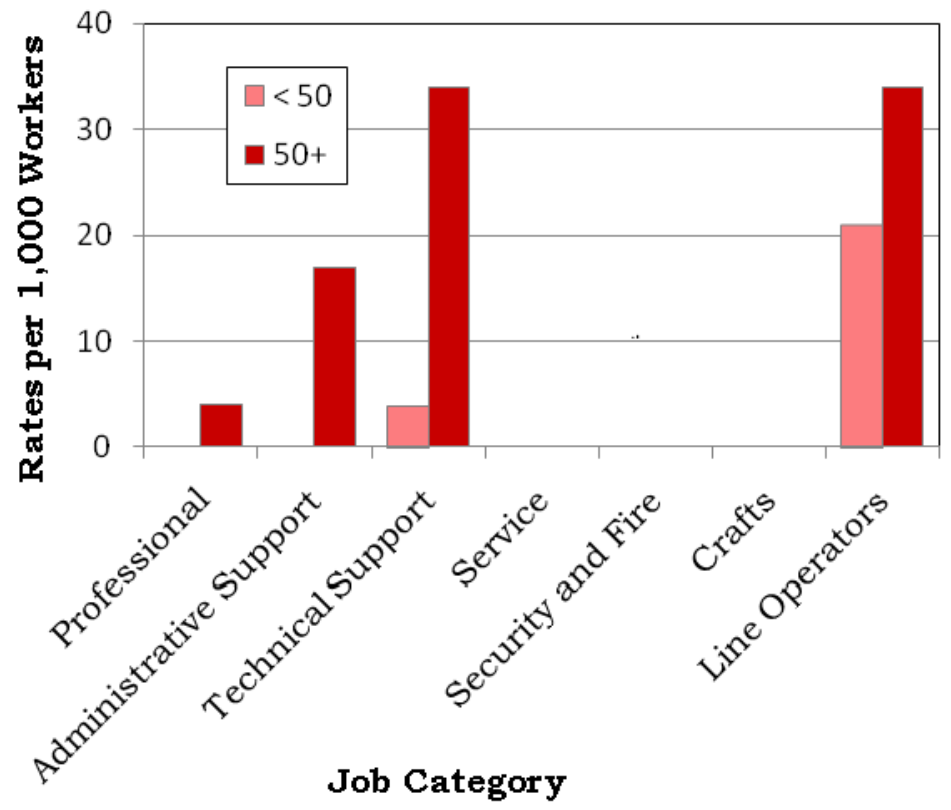

Figure 23. OSHA-Recordable Rates by Age and Job Categories Among Men, All Diagnoses Combined

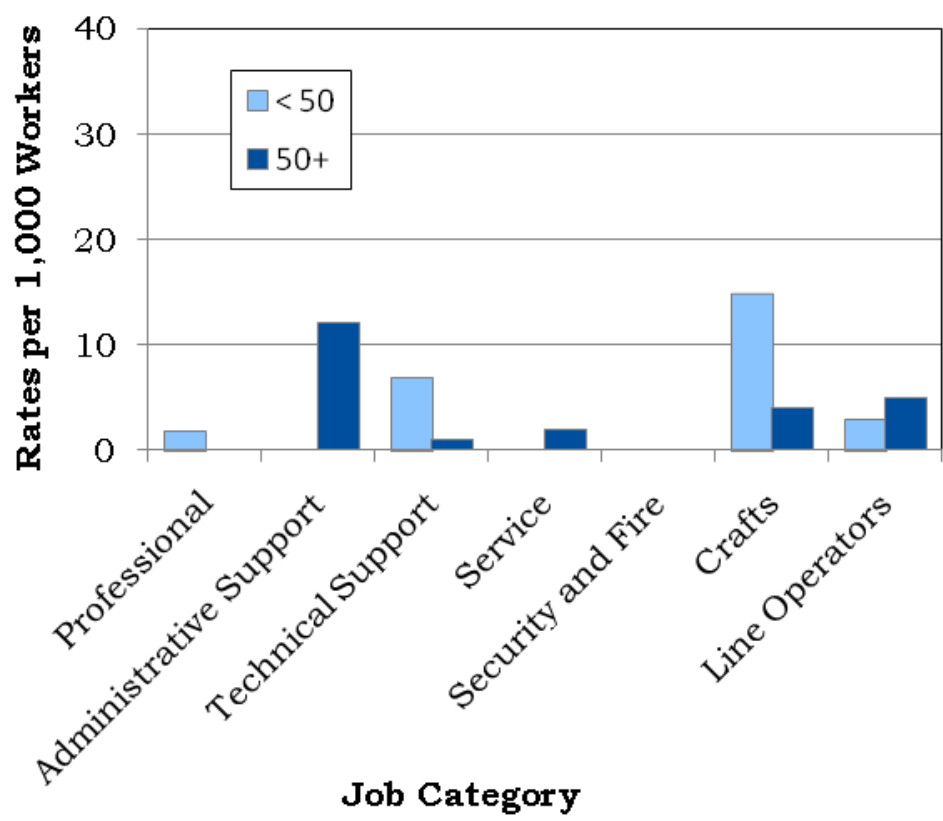




\section{Time Trends for OSHA-Recordable Events}

Figure 24. Age-Adjusted Rates for All OSHA-Recordable Diagnoses Combined Among Women and Men by Job Category from 2003 to 2008*

Professional

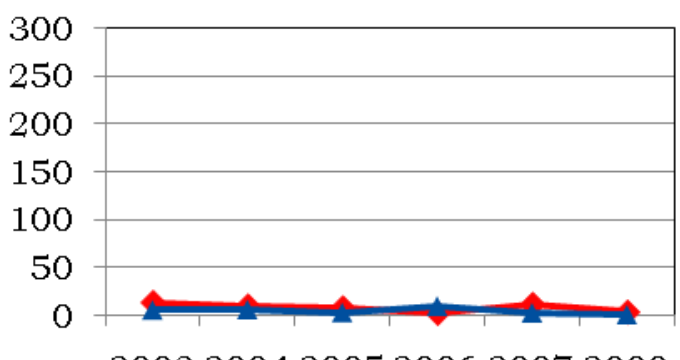

200320042005200620072008

Administrative Support

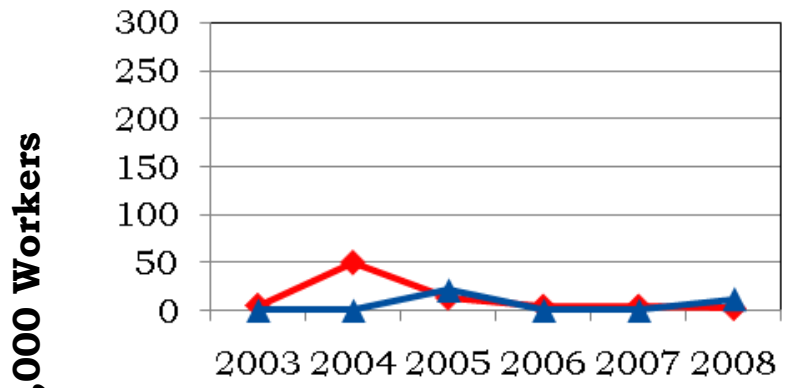

Service

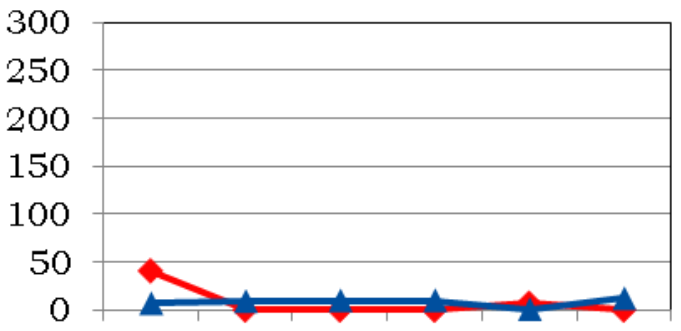

200320042005200620072008

Crafts

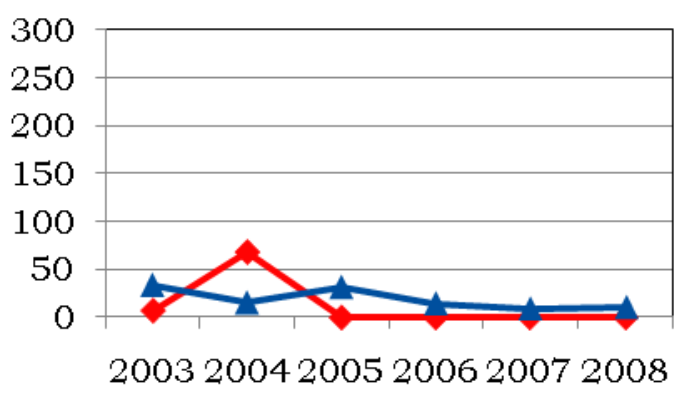

$\leadsto$ Women Men

Technical Support

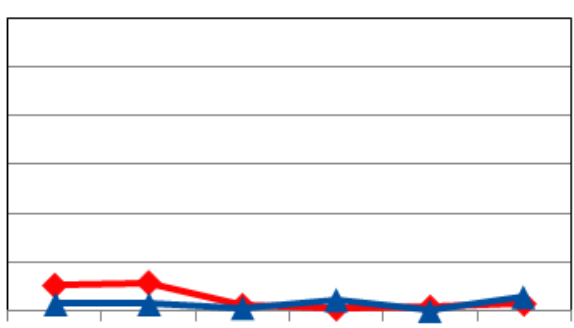

200320042005200620072008

Security and Fire

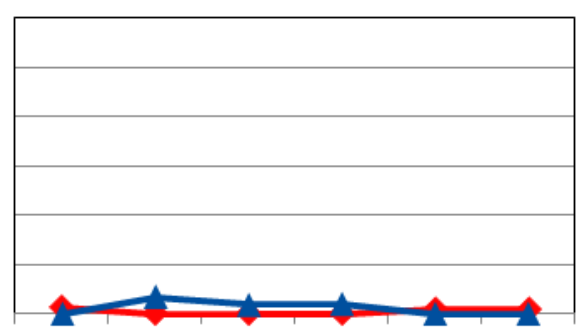

200320042005200620072008

Line Operators

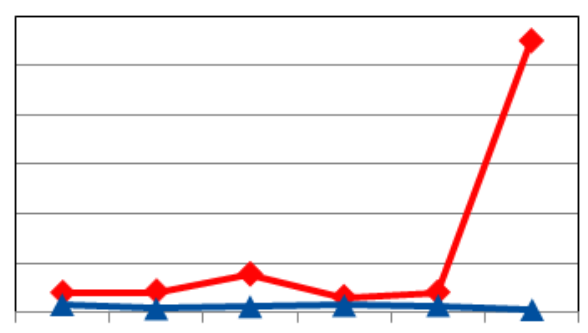

200320042005200620072008

Year

*Standardized to age distribution of 2000 U.S. population. 
Appendices 
Savannah River Site 2008

Absence Data

Appendix A. Work Force by Gender, Age, and Job Category

\begin{tabular}{|c|c|c|c|c|c|c|c|c|c|c|c|}
\hline \multirow{3}{*}{ Job Category } & \multicolumn{5}{|c|}{ Women } & \multicolumn{5}{|c|}{ Men } & \multirow[b]{3}{*}{ TOTAL } \\
\hline & \multicolumn{4}{|c|}{ Age Group } & \multirow[b]{2}{*}{ TOTAL } & \multicolumn{4}{|c|}{ Age Group } & \multirow[b]{2}{*}{ TOTAL } & \\
\hline & $16-29$ & 30 - 39 & $40-49$ & $50+$ & & $16-29$ & 30 - 39 & $40-49$ & $50+$ & & \\
\hline Professional & 19 & 35 & 289 & 266 & 609 & 20 & 89 & 818 & 1,240 & 2,167 & 2,776 \\
\hline Administrative Support & 61 & 20 & 228 & 299 & 608 & 38 & 13 & 91 & 161 & 303 & 911 \\
\hline Technical Support & 7 & 30 & 227 & 234 & 498 & 20 & 47 & 532 & 756 & 1,355 & 1,853 \\
\hline Service & 26 & 31 & 76 & 40 & 173 & 115 & 107 & 420 & 626 & 1,268 & 1,441 \\
\hline Security and Fire & 0 & 0 & 1 & 2 & 3 & 1 & 3 & 19 & 8 & 31 & 34 \\
\hline Crafts & 27 & 16 & 41 & 37 & 121 & 110 & 122 & 223 & 262 & 717 & 838 \\
\hline Line Operators & 1 & 14 & 128 & 149 & 292 & 6 & 40 & 640 & 738 & 1,424 & 1,716 \\
\hline TOTAL & 141 & 146 & 990 & 1,027 & 2,304 & 310 & 421 & 2,743 & 3,791 & 7,265 & 9,569 \\
\hline
\end{tabular}

Appendix B. Age Distribution of the Work Force by Gender

\begin{tabular}{|l|r|r|r|r|r|r|r|r|}
\hline \multirow{3}{*}{ Year } & \multicolumn{9}{|c|}{ Women } & \multicolumn{4}{c|}{ Men } \\
\cline { 2 - 9 } & \multicolumn{1}{|c|}{ Percent Distribution by Age Group } & \multicolumn{1}{c|}{ Percent Distribution by Age Group } \\
\cline { 2 - 9 } & $\mathbf{1 6}-\mathbf{2 9}$ & $\mathbf{3 0}-\mathbf{3 9}$ & $\mathbf{4 0}-\mathbf{4 9}$ & $\mathbf{5 0}+$ & $\mathbf{1 6}-\mathbf{2 9}$ & $\mathbf{3 0}-\mathbf{3 9}$ & $\mathbf{4 0}-\mathbf{4 9}$ & $\mathbf{5 0}+$ \\
\hline $\mathbf{1 9 9 4}$ & 23.92 & 41.93 & 26.45 & 7.71 & 16.20 & 37.97 & 31.48 & 14.35 \\
\hline $\mathbf{1 9 9 5}$ & 18.31 & 42.58 & 29.86 & 9.25 & 11.66 & 37.77 & 34.26 & 16.31 \\
\hline $\mathbf{1 9 9 6}$ & 12.75 & 41.43 & 34.53 & 11.29 & 7.89 & 35.53 & 38.82 & 17.76 \\
\hline $\mathbf{1 9 9 7}$ & 9.53 & 38.76 & 38.48 & 13.23 & 5.51 & 33.40 & 41.02 & 20.06 \\
\hline $\mathbf{1 9 9 8}$ & 8.59 & 35.79 & 40.45 & 15.17 & 4.72 & 30.95 & 42.36 & 21.97 \\
\hline $\mathbf{1 9 9 9}$ & 6.30 & 33.07 & 42.76 & 17.87 & 3.87 & 28.08 & 42.83 & 25.22 \\
\hline $\mathbf{2 0 0 0}$ & 5.49 & 30.09 & 43.31 & 21.10 & 4.19 & 24.74 & 42.87 & 28.20 \\
\hline $\mathbf{2 0 0 1}$ & 5.97 & 26.63 & 43.88 & 23.52 & 4.86 & 22.01 & 43.32 & 29.81 \\
\hline $\mathbf{2 0 0 2}$ & 6.16 & 23.32 & 44.12 & 26.40 & 5.14 & 18.68 & 42.78 & 33.41 \\
\hline $\mathbf{2 0 0 3}$ & 5.22 & 16.88 & 50.33 & 27.57 & 4.93 & 13.71 & 46.65 & 34.70 \\
\hline $\mathbf{2 0 0 4}$ & 4.57 & 14.05 & 49.66 & 31.72 & 4.27 & 11.08 & 45.70 & 38.94 \\
\hline $\mathbf{2 0 0 5}$ & 3.22 & 11.65 & 44.16 & 40.98 & 3.03 & 9.45 & 39.27 & 48.25 \\
\hline $\mathbf{2 0 0 6}$ & 2.62 & 9.82 & 45.53 & 42.04 & 2.74 & 8.71 & 39.43 & 49.13 \\
\hline $\mathbf{2 0 0 7}$ & 5.22 & 7.33 & 47.81 & 39.63 & 4.02 & 6.37 & 42.47 & 47.13 \\
\hline $\mathbf{2 0 0 8}$ & 6.12 & 6.34 & 42.97 & 44.57 & 4.27 & 5.79 & 37.76 & 52.18 \\
\hline
\end{tabular}


Savannah River Site 2008

Absence Data

Appendix C. Total Number of Workers Who Reported at Least One Absence by Gender, Age, and Job Category*

\begin{tabular}{|c|c|c|c|c|c|c|c|c|c|c|c|}
\hline \multirow{3}{*}{ Job Category } & \multicolumn{5}{|c|}{ Women } & \multicolumn{5}{|c|}{ Men } & \multirow[b]{3}{*}{ TOTAL } \\
\hline & \multicolumn{4}{|c|}{ Age Group } & \multirow[b]{2}{*}{ TOTAL } & \multicolumn{4}{|c|}{ Age Group } & \multirow[b]{2}{*}{ TOTAL } & \\
\hline & $16-29$ & 30 - 39 & $40-49$ & $50+$ & & $16-29$ & 30 - 39 & $40-49$ & $50+$ & & \\
\hline Professional & 3 & 7 & 70 & 70 & 150 & 0 & 10 & 133 & 238 & 381 & 531 \\
\hline Administrative Support & 5 & 4 & 67 & 93 & 169 & 1 & 1 & 16 & 28 & 46 & 215 \\
\hline Technical Support & 2 & 15 & 97 & 98 & 212 & 5 & 10 & 121 & 190 & 326 & 538 \\
\hline Service & 2 & 9 & 14 & 7 & 32 & 7 & 13 & 48 & 91 & 159 & 191 \\
\hline Security and Fire & 0 & 0 & 0 & 0 & 0 & 0 & 1 & 4 & 1 & 6 & 6 \\
\hline Crafts & 5 & 2 & 1 & 1 & 9 & 5 & 5 & 14 & 25 & 49 & 58 \\
\hline Line Operators & 1 & 10 & 82 & 71 & 164 & 4 & 17 & 276 & 293 & 590 & 754 \\
\hline TOTAL & 18 & 47 & 331 & 340 & 736 & 22 & 57 & 612 & 866 & 1,557 & 2,293 \\
\hline
\end{tabular}

*Only those job categories and gender/age combinations with at least one absence appear in this table.

Appendix D. Total Number of Absences by Gender, Age, and Job Category*

\begin{tabular}{|c|c|c|c|c|c|c|c|c|c|c|c|}
\hline \multirow{3}{*}{ Job Category } & \multicolumn{5}{|c|}{ Women } & \multicolumn{5}{|c|}{ Men } & \multirow[b]{3}{*}{ TOTAL } \\
\hline & \multicolumn{4}{|c|}{ Age Group } & \multirow[b]{2}{*}{ TOTAL } & \multicolumn{4}{|c|}{ Age Group } & \multirow[b]{2}{*}{ TOTAL } & \\
\hline & $16-29$ & 30 - 39 & $40-49$ & $50+$ & & $16-29$ & 30 - 39 & $40-49$ & $50+$ & & \\
\hline Professional & 4 & 17 & 108 & 104 & 233 & 0 & 12 & 188 & 369 & 569 & 802 \\
\hline Administrative Support & 7 & 7 & 98 & 147 & 259 & 1 & 1 & 24 & 43 & 69 & 328 \\
\hline Technical Support & 4 & 40 & 199 & 200 & 443 & 7 & 19 & 184 & 309 & 519 & 962 \\
\hline Service & 2 & 16 & 26 & 9 & 53 & 8 & 16 & 80 & 112 & 216 & 269 \\
\hline Security and Fire & 0 & 0 & 0 & 0 & 0 & 0 & 1 & 4 & 1 & 6 & 6 \\
\hline Crafts & 8 & 4 & 2 & 1 & 15 & 5 & 6 & 17 & 29 & 57 & 72 \\
\hline Line Operators & 1 & 18 & 146 & 173 & 338 & 6 & 30 & 486 & 509 & 1,031 & 1,369 \\
\hline TOTAL & 26 & 102 & 579 & 634 & 1,341 & 27 & 85 & 983 & 1,372 & 2,467 & 3,808 \\
\hline
\end{tabular}

*Only those job categories and gender/age combinations with at least one absence appear in this table. 
Savannah River Site 2008

Absence Data

Appendix E. Distribution of the Number of Calendar Days Missed per Absence by Gender and Age*

\begin{tabular}{|c|c|c|c|c|c|c|c|c|c|c|c|}
\hline \multirow{3}{*}{$\begin{array}{c}\text { Number of } \\
\text { Calendar Days }\end{array}$} & \multicolumn{5}{|c|}{ Women } & \multicolumn{5}{|c|}{ Men } & \multirow[b]{3}{*}{ TOTAL } \\
\hline & \multicolumn{4}{|c|}{ Age Group } & \multirow[b]{2}{*}{ TOTAL } & \multicolumn{4}{|c|}{ Age Group } & \multirow[b]{2}{*}{ TOTAL } & \\
\hline & $16-29$ & 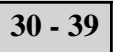 & 40 - 49 & $50+$ & & $16-29$ & 30 - 39 & $40-49$ & $50+$ & & \\
\hline$<15$ & 20 & 77 & 415 & 461 & 973 & 21 & 64 & 753 & 964 & 1,802 & 2,775 \\
\hline $15-28$ & 6 & 13 & 73 & 93 & 185 & 3 & 13 & 138 & 209 & 363 & 548 \\
\hline $29-42$ & 0 & 6 & 35 & 24 & 65 & 1 & 4 & 38 & 69 & 112 & 177 \\
\hline $43-56$ & 0 & 5 & 30 & 26 & 61 & 0 & 0 & 19 & 53 & 72 & 133 \\
\hline 57 - 91 & 0 & 1 & 13 & 13 & 27 & 2 & 1 & 21 & 44 & 68 & 95 \\
\hline $92-182$ & 0 & 0 & 9 & 13 & 22 & 0 & 1 & 9 & 26 & 36 & 58 \\
\hline $183+$ & 0 & 0 & 4 & 4 & 8 & 0 & 2 & 5 & 7 & 14 & 22 \\
\hline TOTAL & 26 & 102 & 579 & 634 & 1,341 & 27 & 85 & 983 & 1,372 & 2,467 & 3,808 \\
\hline
\end{tabular}

*Only those gender/age combinations with at least one absence appear in this table. 
Savannah River Site 2008

Absence Data

Appendix F. Distribution of the Number of Calendar Days Missed per Absence by Gender and Job Category*

Women

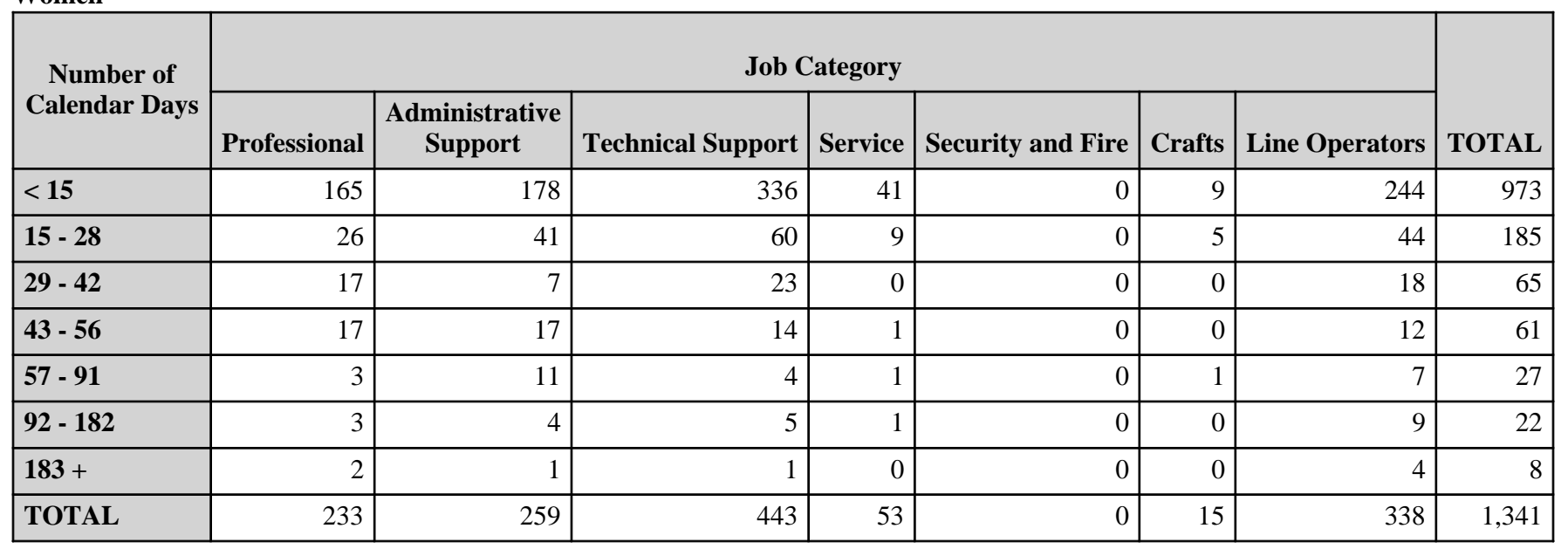

Men

\begin{tabular}{|c|c|c|c|c|c|c|c|c|}
\hline \multirow{2}{*}{$\begin{array}{c}\text { Number of } \\
\text { Calendar Days }\end{array}$} & \multicolumn{7}{|c|}{ Job Category } & \multirow[b]{2}{*}{ TOTAL } \\
\hline & Professional & $\begin{array}{l}\text { Administrative } \\
\text { Support }\end{array}$ & Technical Support & Service & Security and Fire & Crafts & Line Operators & \\
\hline$<15$ & 427 & 46 & 379 & 150 & 2 & 42 & 756 & 1,802 \\
\hline $15-28$ & 70 & 12 & 79 & 36 & 2 & 3 & 161 & 363 \\
\hline $57-91$ & 14 & 0 & 13 & 13 & 1 & 2 & 25 & 68 \\
\hline $92-182$ & 9 & 3 & 7 & 1 & 1 & 5 & 10 & 36 \\
\hline $183+$ & 6 & 1 & 2 & 2 & 0 & 0 & 3 & 14 \\
\hline TOTAL & 569 & 69 & 519 & 216 & 6 & 57 & 1,031 & 2,467 \\
\hline
\end{tabular}

*Only those gender/job category combinations with at least one absence appear in this table. 


\section{Savannah River Site 2008}

\section{Absence Data}

Appendix G. Number of Diagnoses in Each Diagnostic Category by Gender and Age*

\begin{tabular}{|c|c|c|c|c|c|c|}
\hline & & \multicolumn{5}{|c|}{ Women } \\
\hline & & \multicolumn{4}{|c|}{ Age Group } & \multirow[b]{2}{*}{ TOTAL } \\
\hline & & $16-29$ & $30-39$ & $40-49$ & $\mathbf{5 0 +}$ & \\
\hline Diagnostic Category & ICD-9-CM Code & & & & & \\
\hline INFECTIOUS \& PARASITIC DISEASES (DIS) & 001-139 & 1 & 10 & 37 & 22 & 70 \\
\hline -Intestinal Infectious Dis & 001-009 & 0 & 5 & 11 & 9 & 25 \\
\hline -Other Bacterial Dis & 030-041 & 1 & 0 & 10 & 4 & 15 \\
\hline -Viral Dis with Exanthem & 050-057 & 0 & 2 & 4 & 1 & 7 \\
\hline -Arthropod-Borne Viral Dis & $060-066$ & 0 & 0 & 0 & 0 & 0 \\
\hline -Other Viral Dis \& Chlamydiae & 070-079 & 0 & 3 & 5 & 5 & 13 \\
\hline -Rickettsioses \& Other Arthropod & 080-088 & 0 & 0 & 0 & 0 & 0 \\
\hline -Mycoses & $110-118$ & 0 & 0 & 7 & 0 & 7 \\
\hline -Other Infections \& Parasitic Dis & $130-136$ & 0 & 0 & 0 & 3 & 3 \\
\hline MALIGNANT NEOPLASMS & $140-208,230-234$ & 0 & 0 & 19 & 12 & 31 \\
\hline -Lip, Oral Cavity, Pharynx & $140-149$ & 0 & 0 & 0 & 0 & 0 \\
\hline -Digestive \& Peritoneal & $150-159$ & 0 & 0 & 5 & 0 & 5 \\
\hline -Respiratory \& Intrathoracic & $160-165$ & 0 & 0 & 0 & 0 & 0 \\
\hline -Bone, Connective Tissue, Skin & 170-173, 176 & 0 & 0 & 1 & 0 & 1 \\
\hline -Breast & 174-175 & 0 & 0 & 1 & 11 & 12 \\
\hline -Genitourinary & 179-189 & 0 & 0 & 1 & 0 & 1 \\
\hline -Other \& Unspecified Sites & $190,193-199$ & 0 & 0 & 11 & 0 & 11 \\
\hline -Nervous System & 191-192 & 0 & 0 & 0 & 0 & 0 \\
\hline -Lymphatic \& Hematopoietic & $200-208$ & 0 & 0 & 0 & 1 & 1 \\
\hline -Carcinoma in situ & $230-234$ & 0 & 0 & 0 & 0 & 0 \\
\hline BENIGN \& UNCERTAIN NEOPLASMS & 210-229, 235-239 & 0 & 2 & 23 & 9 & 34 \\
\hline ENDOCRINE/METABOLIC/IMMUNITY & 240-279 & 0 & 4 & 22 & 24 & 50 \\
\hline -Thyroid Gland Disorders & $240-246$ & 0 & 1 & 2 & 8 & 11 \\
\hline -Other Endocrine Gland Dis & $250-259$ & 0 & 3 & 8 & 10 & 21 \\
\hline -Nutritional Deficiencies & $260-269$ & 0 & 0 & 2 & 0 & 2 \\
\hline -Other Metabolic \& Immunity Disorders & 270-279 & 0 & 0 & 10 & 6 & 16 \\
\hline BLOOD \& BLOOD-FORMING ORGANS & $280-289$ & 0 & 0 & 25 & 4 & 29 \\
\hline MENTAL DISORDERS & $290-319$ & 0 & 0 & 19 & 13 & 32 \\
\hline -Psychoses & 290-299 & 0 & 0 & 1 & 2 & 3 \\
\hline -Non-Psychotic Disorders & $300-302,306-316$ & 0 & 0 & 14 & 11 & 25 \\
\hline -Alcohol Dependence & 303 & 0 & 0 & 2 & 0 & 2 \\
\hline -Drug Dependence & $304-305$ & 0 & 0 & 2 & 0 & 2 \\
\hline NERVOUS SYSTEM (NS) \& SENSE ORGANS & 320-389 & 2 & 13 & 56 & 67 & 138 \\
\hline -Hereditary/Degenerative Central NS Dis & 330-337 & 0 & 0 & 1 & 0 & 1 \\
\hline -Other Disorders of Central NS & $340-349$ & 0 & 2 & 24 & 8 & 34 \\
\hline -Disorders of Peripheral NS & 350-359 & 0 & 0 & 11 & 13 & 24 \\
\hline -Disorders of Eye & $360-379$ & 0 & 8 & 13 & 30 & 51 \\
\hline
\end{tabular}

*Only those diagnostic categories and gender/age combinations with at least one occurrence appear in this table. 


\section{Savannah River Site 2008}

\section{Absence Data}

Appendix G. Number of Diagnoses in Each Diagnostic Category by Gender and Age*

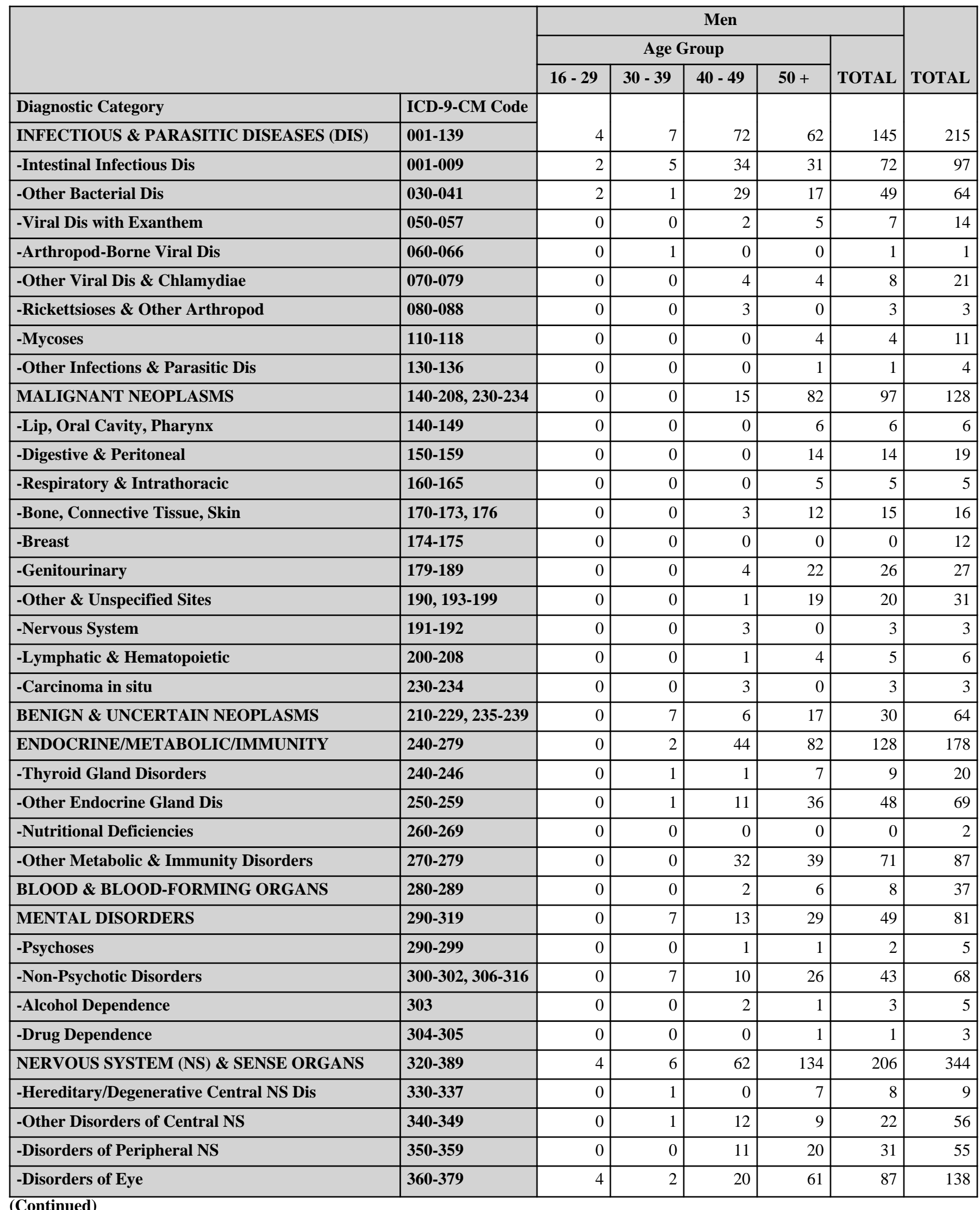

*Only those diagnostic categories and gender/age combinations with at least one occurrence appear in this table. 


\section{Savannah River Site 2008}

\section{Absence Data}

Appendix G. Number of Diagnoses in Each Diagnostic Category by Gender and Age*

\begin{tabular}{|c|c|c|c|c|c|c|}
\hline & & \multicolumn{5}{|c|}{ Women } \\
\hline & & \multicolumn{4}{|c|}{ Age Group } & \multirow[b]{2}{*}{ TOTAL } \\
\hline & & $16-29$ & 30 - 39 & 40 - 49 & $50+$ & \\
\hline Diagnostic Category & ICD-9-CM Code & \multirow[b]{2}{*}{2} & \multirow[b]{2}{*}{3} & \multirow[b]{2}{*}{7} & \multirow[b]{2}{*}{16} & \multirow[b]{2}{*}{28} \\
\hline -Dis of Ear \& Mastoid & $380-389$ & & & & & \\
\hline CIRCULATORY SYSTEM & $390-459$ & 0 & 10 & 31 & 67 & 108 \\
\hline -Hypertensive Dis & $401-405$ & 0 & 4 & 28 & 25 & 57 \\
\hline -Ischemic Heart Dis & $410-414$ & 0 & 0 & 0 & 2 & 2 \\
\hline -Dis of Pulmonary Circulation & $415-417$ & 0 & 0 & 0 & 2 & 2 \\
\hline -Other Heart Dis & $420-429$ & 0 & 0 & 0 & 18 & 18 \\
\hline -Cerebrovascular Dis & $430-438$ & 0 & 0 & 0 & 2 & 2 \\
\hline -Dis of Arteries \& Capillaries & $440-448$ & 0 & 0 & 0 & 0 & 0 \\
\hline -Dis of Veins, Lymphatics, Other & 451-459 & 0 & 6 & 3 & 18 & 27 \\
\hline RESPIRATORY SYSTEM & $460-519$ & 7 & 46 & 205 & 272 & 530 \\
\hline -Acute Respiratory Infections & $460-466$ & 1 & 25 & 99 & 122 & 247 \\
\hline -Other Dis Upper Respiratory Tract & $470-478$ & 4 & 13 & 50 & 73 & 140 \\
\hline -Pneumonia \& Influenza & $480-487$ & 2 & 4 & 46 & 56 & 108 \\
\hline -Chronic Obstructive Dis & $490-496$ & 0 & 4 & 6 & 9 & 19 \\
\hline -Lung Dis from External Agents & $500-508$ & 0 & 0 & 1 & 2 & 3 \\
\hline -Other Respiratory Dis & $510-519$ & 0 & 0 & 3 & 10 & 13 \\
\hline DIGESTIVE SYSTEM & $520-579$ & 3 & 14 & 73 & 63 & 153 \\
\hline -Oral Cavity, Saliva Glands, Jaw & $520-529$ & 0 & 0 & 25 & 13 & 38 \\
\hline -Esophagus, Stomach, Duodenum & $530-537$ & 0 & 3 & 8 & 12 & 23 \\
\hline -Appendicitis & $540-543$ & 0 & 0 & 1 & 0 & 1 \\
\hline -Hernia & $550-553$ & 0 & 3 & 4 & 6 & 13 \\
\hline -Enteritis, Colitis & $555-558$ & 2 & 0 & 10 & 2 & 14 \\
\hline -Other Intestinal Dis & $560-569$ & 0 & 5 & 5 & 9 & 19 \\
\hline -Other Digestive Dis & $570-579$ & 1 & 3 & 20 & 21 & 45 \\
\hline GENITOURINARY SYSTEM & $580-629$ & 8 & 18 & 122 & 51 & 199 \\
\hline -Nephritis, Nephrosis & $580-589$ & 0 & 0 & 2 & 6 & 8 \\
\hline -Other Urinary Dis & $590-599$ & 1 & 4 & 38 & 18 & 61 \\
\hline -Male Genital Organ Dis & $600-608$ & 0 & 0 & 0 & 0 & 0 \\
\hline -Breast Disorders & $610-611$ & 0 & 0 & 4 & 2 & 6 \\
\hline -Pelvic Inflammatory Dis & 614-616 & 0 & 5 & 9 & 2 & 16 \\
\hline -Other Female Disorders & $617-629$ & 7 & 9 & 69 & 23 & 108 \\
\hline PREGNANCY \& CHILDBIRTH & $630-677$ & 0 & 4 & 0 & 0 & 4 \\
\hline -Pregnancy with Abortive Outcome & $634-639$ & 0 & 4 & 0 & 0 & 4 \\
\hline SKIN \& SUBCUTANEOUS TISSUE & $680-709$ & 0 & 4 & 13 & 6 & 23 \\
\hline -Infections & $680-686$ & 0 & 1 & 6 & 3 & 10 \\
\hline -Other Inflammatory Conditions & $690-698$ & 0 & 0 & 0 & 0 & 0 \\
\hline -Other & 700-709 & 0 & 3 & 7 & 3 & 13 \\
\hline
\end{tabular}

*Only those diagnostic categories and gender/age combinations with at least one occurrence appear in this table. 


\section{Savannah River Site 2008}

\section{Absence Data}

Appendix G. Number of Diagnoses in Each Diagnostic Category by Gender and Age*

\begin{tabular}{|c|c|c|c|c|c|c|c|}
\hline & & \multicolumn{5}{|c|}{ Men } & \multirow[b]{3}{*}{ TOTAL } \\
\hline & & \multicolumn{4}{|c|}{ Age Group } & \multirow[b]{2}{*}{ TOTAL } & \\
\hline & & $16-29$ & $30-39$ & $40-49$ & $50+$ & & \\
\hline -Dis of Ear \& Mastoid & $380-389$ & 0 & 2 & 19 & 33 & 54 & 82 \\
\hline CIRCULATORY SYSTEM & $390-459$ & 1 & 2 & 101 & 173 & 277 & 385 \\
\hline -Ischemic Heart Dis & $410-414$ & 0 & 0 & 23 & 41 & 64 & 66 \\
\hline -Dis of Pulmonary Circulation & $415-417$ & 0 & 0 & 0 & 1 & 1 & 3 \\
\hline -Other Heart Dis & $420-429$ & 0 & 0 & 18 & 18 & 36 & 54 \\
\hline -Cerebrovascular Dis & $430-438$ & 1 & 1 & 7 & 9 & 18 & 20 \\
\hline -Acute Respiratory Infections & $460-466$ & 5 & 11 & 152 & 200 & 368 & 615 \\
\hline -Other Dis Upper Respiratory Tract & $470-478$ & 3 & 18 & 125 & 111 & 257 & 397 \\
\hline -Pneumonia \& Influenza & $480-487$ & 1 & 8 & 105 & 137 & 251 & 359 \\
\hline -Chronic Obstructive Dis & $490-496$ & 0 & 0 & 6 & 8 & 14 & 33 \\
\hline -Lung Dis from External Agents & $500-508$ & 0 & 0 & 1 & 6 & 7 & 10 \\
\hline -Other Respiratory Dis & $510-519$ & 0 & 0 & 1 & 8 & 9 & 22 \\
\hline DIGESTIVE SYSTEM & $520-579$ & 1 & 3 & 97 & 231 & 332 & 485 \\
\hline -Oral Cavity, Saliva Glands, Jaw & $520-529$ & 0 & 1 & 8 & 28 & 37 & 75 \\
\hline -Other Digestive Dis & $570-579$ & 0 & 0 & 20 & 21 & 41 & 86 \\
\hline GENITOURINARY SYSTEM & $580-629$ & 1 & 0 & 65 & 121 & 187 & 386 \\
\hline -Nephritis, Nephrosis & $580-589$ & 0 & 0 & 0 & 8 & 8 & 16 \\
\hline -Other Urinary Dis & $590-599$ & 1 & 0 & 59 & 75 & 135 & 196 \\
\hline -Male Genital Organ Dis & $600-608$ & 0 & 0 & 5 & 37 & 42 & 42 \\
\hline -Breast Disorders & $610-611$ & 0 & 0 & 1 & 1 & 2 & 8 \\
\hline -Pelvic Inflammatory Dis & 614-616 & 0 & 0 & 0 & 0 & 0 & 16 \\
\hline -Other Female Disorders & $617-629$ & 0 & 0 & 0 & 0 & 0 & 108 \\
\hline PREGNANCY \& CHILDBIRTH & $630-677$ & 0 & 0 & 0 & 0 & 0 & 4 \\
\hline -Pregnancy with Abortive Outcome & $634-639$ & 0 & 0 & 0 & 0 & 0 & 4 \\
\hline SKIN \& SUBCUTANEOUS TISSUE & $680-709$ & 1 & 1 & 15 & 26 & 43 & 66 \\
\hline -Infections & $680-686$ & 1 & 0 & 4 & 5 & 10 & 20 \\
\hline -Other Inflammatory Conditions & $690-698$ & 0 & 0 & 1 & 10 & 11 & 11 \\
\hline -Other & 700-709 & 0 & 1 & 10 & 11 & 22 & 35 \\
\hline
\end{tabular}

*Only those diagnostic categories and gender/age combinations with at least one occurrence appear in this table. 


\section{Savannah River Site 2008}

\section{Absence Data}

Appendix G. Number of Diagnoses in Each Diagnostic Category by Gender and Age*

\begin{tabular}{|c|c|c|c|c|c|c|}
\hline & & \multicolumn{5}{|c|}{ Women } \\
\hline & & \multicolumn{4}{|c|}{ Age Group } & \multirow[b]{2}{*}{ TOTAL } \\
\hline & & $16-29$ & $30-39$ & $40-49$ & $\mathbf{5 0 +}$ & \\
\hline Diagnostic Category & ICD-9-CM Code & & & & & \\
\hline $\begin{array}{l}\text { MUSCULOSKELETAL \& CONNECTIVE } \\
\text { TISSUE }\end{array}$ & $710-739$ & 7 & 33 & 121 & 278 & 439 \\
\hline -Arthropathies & $710-719$ & 3 & 0 & 15 & 60 & 78 \\
\hline -Dorsopathies & $720-724$ & 3 & 31 & 73 & 165 & 272 \\
\hline -Rheumatism, Excluding Back & $725-729$ & 1 & 2 & 25 & 45 & 73 \\
\hline -Other Dis \& Acquired Deformities & $730-739$ & 0 & 0 & 8 & 8 & 16 \\
\hline CONGENITAL ANOMALIES & $740-759$ & 0 & 0 & 0 & 0 & 0 \\
\hline $\begin{array}{l}\text { SYMPTOMS, SIGNS, \& ILL-DEFINED } \\
\text { CONDITIONS }\end{array}$ & $780-799$ & 4 & 6 & 122 & 110 & 242 \\
\hline -Symptoms & $780-789$ & 4 & 6 & 119 & 103 & 232 \\
\hline -Non-Specific Abnormal Findings & $790-796$ & 0 & 0 & 3 & 5 & 8 \\
\hline -IIl-Defined \& Unknown Causes & 797-799 & 0 & 0 & 0 & 2 & 2 \\
\hline INJURY \& POISONING & $800-999$ & 3 & 8 & 61 & 77 & 149 \\
\hline -Fracture - Skull & 800-804 & 0 & 0 & 0 & 0 & 0 \\
\hline -Fracture - Neck, Trunk & $805-809$ & 0 & 0 & 1 & 6 & 7 \\
\hline -Fracture - Upper Limb & 810-819 & 2 & 0 & 2 & 9 & 13 \\
\hline -Fracture - Lower Limb & $820-829$ & 0 & 1 & 8 & 8 & 17 \\
\hline -Dislocation & $830-839$ & 0 & 0 & 4 & 6 & 10 \\
\hline -Sprains \& Strains - Back & 846-847 & 0 & 1 & 9 & 9 & 19 \\
\hline -Sprains \& Strains - Other & $840-845,848$ & 0 & 0 & 8 & 8 & 16 \\
\hline -Intracranial Injury & $850-854$ & 0 & 0 & 0 & 2 & 2 \\
\hline -Internal Injury - Thorax, Abdomen, Pelvis & $860-869$ & 0 & 0 & 0 & 0 & 0 \\
\hline -Open Wound - Head, Neck, Trunk & $870-879$ & 0 & 1 & 6 & 2 & 9 \\
\hline -Open Wound - Upper Limb & 880-887 & 0 & 0 & 0 & 0 & 0 \\
\hline -Open Wound - Lower Limb & $890-897$ & 0 & 0 & 0 & 1 & 1 \\
\hline -Late Effects of Accident & $905-909$ & 0 & 0 & 0 & 0 & 0 \\
\hline -Superficial Injury & $910-919$ & 0 & 2 & 1 & 1 & 4 \\
\hline -Contusion & $920-924$ & 0 & 1 & 7 & 10 & 18 \\
\hline -Foreign Body Entering Orifice & 930-939 & 0 & 0 & 0 & 0 & 0 \\
\hline -Burns & $940-949$ & 0 & 0 & 0 & 0 & 0 \\
\hline -Injury to Nerves \& Spinal Cord & $950-957$ & 0 & 0 & 0 & 0 & 0 \\
\hline -Complications \& Unspecified Injuries & 958-959 & 0 & 0 & 0 & 0 & 0 \\
\hline -Poisoning - Medicinal/Biological & $960-979$ & 0 & 0 & 0 & 0 & 0 \\
\hline -Unspecified Effects - External Causes & $990-995$ & 1 & 0 & 7 & 1 & 9 \\
\hline -Complications of Surgical/Medical Care & 996-999 & 0 & 2 & 8 & 14 & 24 \\
\hline $\begin{array}{l}\text { HEALTH STATUS/HEALTH SERVICE } \\
\text { CONTACT }\end{array}$ & V01-V82 & 2 & 8 & 34 & 39 & 83 \\
\hline -Isolation \& Prophylactic Measures & V07-V09 & 0 & 0 & 2 & 0 & 2 \\
\hline
\end{tabular}

(Continued)

*Only those diagnostic categories and gender/age combinations with at least one occurrence appear in this table. 


\section{Savannah River Site 2008}

\section{Absence Data}

Appendix G. Number of Diagnoses in Each Diagnostic Category by Gender and Age*

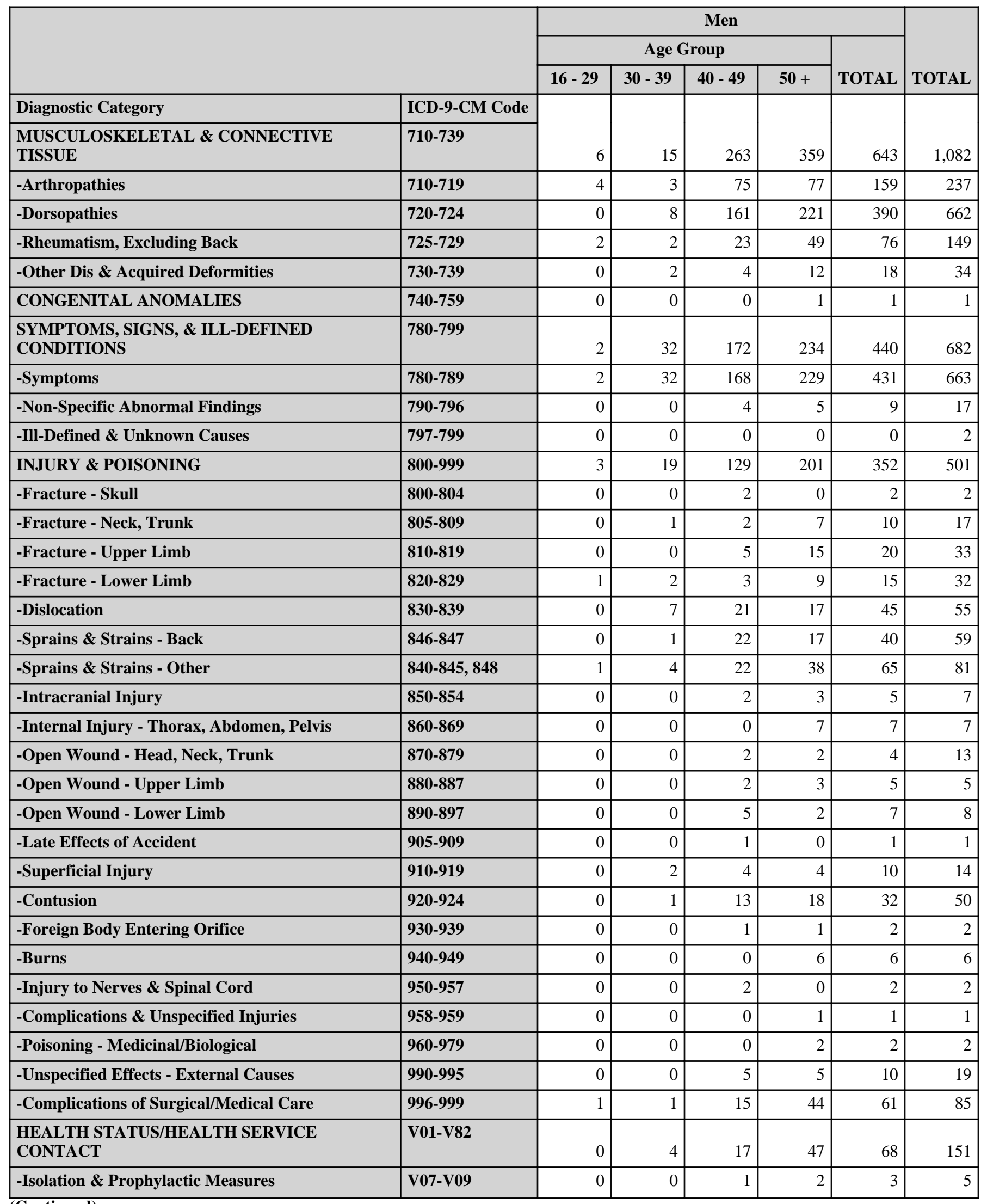

(Continued)

*Only those diagnostic categories and gender/age combinations with at least one occurrence appear in this table. 


\section{Savannah River Site 2008}

\section{Absence Data}

Appendix G. Number of Diagnoses in Each Diagnostic Category by Gender and Age*

\begin{tabular}{|c|c|c|c|c|c|c|}
\hline & & \multicolumn{5}{|c|}{ Women } \\
\hline & & \multicolumn{4}{|c|}{ Age Group } & \multirow[b]{2}{*}{ TOTAL } \\
\hline & & $16-29$ & $30-39$ & $40-49$ & $50+$ & \\
\hline Diagnostic Category & ICD-9-CM Code & \multirow[b]{2}{*}{0} & \multirow[b]{2}{*}{0} & \multirow[b]{2}{*}{7} & \multirow[b]{2}{*}{1} & \multirow[b]{2}{*}{8} \\
\hline -Personal \& Family History & V10-V19 & & & & & \\
\hline -Health Services Reproduction/Development & V20-V29 & 2 & 8 & 9 & 0 & 19 \\
\hline -Health Status & V40-V49 & 0 & 0 & 5 & 9 & 14 \\
\hline -Specific Procedures/Aftercare & V50-V59 & 0 & 0 & 11 & 26 & 37 \\
\hline -Other Circumstances & V60-V69 & 0 & 0 & 0 & 0 & 0 \\
\hline -Examination \& Investigation & V70-V82 & 0 & 0 & 0 & 3 & 3 \\
\hline
\end{tabular}

*Only those diagnostic categories and gender/age combinations with at least one occurrence appear in this table. 


\section{Savannah River Site 2008}

\section{Absence Data}

Appendix G. Number of Diagnoses in Each Diagnostic Category by Gender and Age*

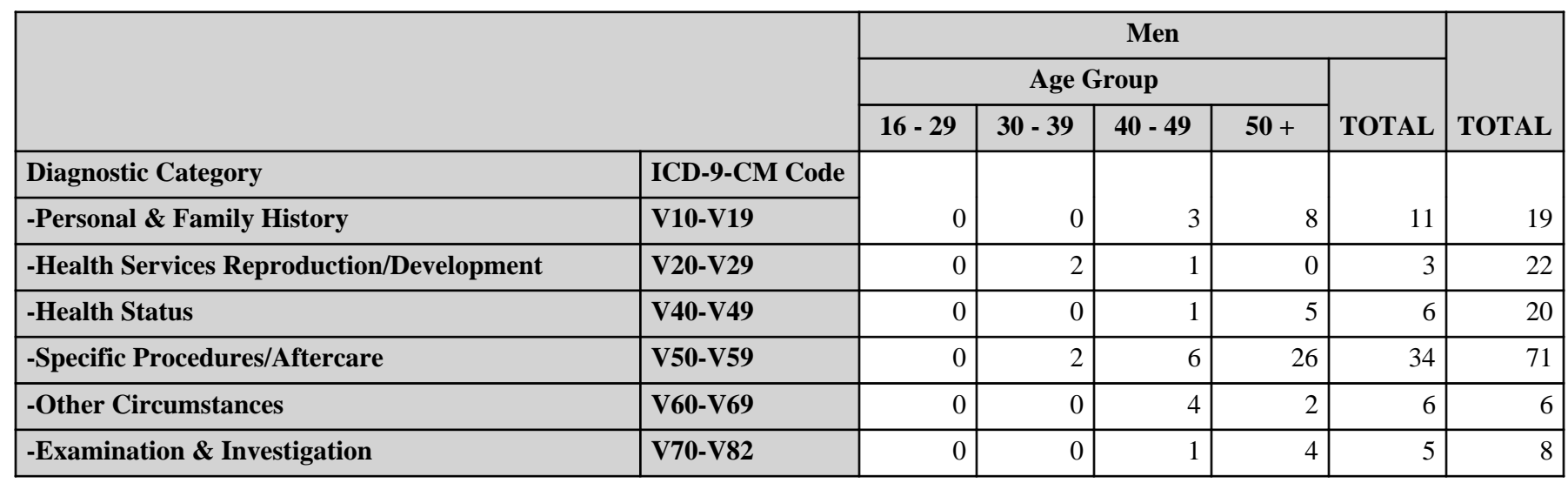

\begin{tabular}{|c|c|c|c|c|c|c|c|c|c|c|c|}
\hline & \multicolumn{5}{|c|}{ Women } & \multicolumn{5}{|c|}{ Men } & \multirow[b]{3}{*}{ TOTAL } \\
\hline & \multicolumn{4}{|c|}{ Age Group } & \multirow[b]{2}{*}{ TOTAL } & \multicolumn{4}{|c|}{ Age Group } & \multirow[b]{2}{*}{ TOTAL } & \\
\hline & $16-29$ & $30-39$ & $40-49$ & $50+$ & & $16-29$ & $30-39$ & $40-49$ & $50+$ & & \\
\hline Diagnostic Category & \multirow[b]{2}{*}{37} & \multirow[b]{2}{*}{180} & \multirow[b]{2}{*}{983} & \multirow[b]{2}{*}{1,114} & \multirow[b]{2}{*}{2,314} & \multirow[b]{2}{*}{32} & \multirow[b]{2}{*}{142} & \multirow[b]{2}{*}{1,463} & \multirow[b]{2}{*}{2,275} & \multirow[b]{2}{*}{3,912} & \multirow[b]{2}{*}{6,226} \\
\hline Total & & & & & & & & & & & \\
\hline
\end{tabular}

*Only those diagnostic categories and gender/age combinations with at least one occurrence appear in this table. 
Savannah River Site 2008

Absence Data

Appendix H. Total Number of Calendar Days Absent in Each Diagnostic Category by Gender and Age*

\begin{tabular}{|c|c|c|c|c|c|c|}
\hline & & \multicolumn{5}{|c|}{ Women } \\
\hline & & \multicolumn{4}{|c|}{ Age Group } & \multirow[b]{2}{*}{ TOTAL } \\
\hline & & $16-29$ & $30-39$ & $40-49$ & $50+$ & \\
\hline Diagnostic Category & ICD-9-CM Code & \multirow[b]{2}{*}{20} & \multirow[b]{2}{*}{70} & \multirow[b]{2}{*}{363} & \multirow[b]{2}{*}{209} & \multirow[b]{2}{*}{662} \\
\hline INFECTIOUS \& PARASITIC DISEASES (DIS) & $001-139$ & & & & & \\
\hline MALIGNANT NEOPLASMS & $140-208,230-234$ & 0 & 0 & 303 & 480 & 783 \\
\hline BENIGN \& UNCERTAIN NEOPLASMS & 210-229, 235-239 & 0 & 33 & 763 & 223 & 1,019 \\
\hline ENDOCRINE/METABOLIC/IMMUNITY & $240-279$ & 0 & 54 & 557 & 402 & 1,013 \\
\hline BLOOD \& BLOOD-FORMING ORGANS & $280-289$ & 0 & 0 & 462 & 153 & 615 \\
\hline MENTAL DISORDERS & $290-319$ & 0 & 0 & 205 & 602 & 807 \\
\hline NERVOUS SYSTEM (NS) \& SENSE ORGANS & $320-389$ & 23 & 111 & 779 & 917 & 1,830 \\
\hline CIRCULATORY SYSTEM & $390-459$ & 0 & 63 & 434 & 1,148 & 1,645 \\
\hline RESPIRATORY SYSTEM & $460-519$ & 73 & 422 & 1,991 & 3,021 & 5,507 \\
\hline DIGESTIVE SYSTEM & $520-579$ & 41 & 157 & 1,376 & 1,001 & 2,575 \\
\hline GENITOURINARY SYSTEM & $580-629$ & 65 & 351 & 1,931 & 1,054 & 3,401 \\
\hline PREGNANCY \& CHILDBIRTH & $630-677$ & 0 & 32 & 0 & 0 & 32 \\
\hline SKIN \& SUBCUTANEOUS TISSUE & 680-709 & 0 & 60 & 182 & 80 & 322 \\
\hline $\begin{array}{l}\text { MUSCULOSKELETAL \& CONNECTIVE } \\
\text { TISSUE }\end{array}$ & $710-739$ & 37 & 208 & 2,295 & 3,444 & 5,984 \\
\hline CONGENITAL ANOMALIES & $740-759$ & 0 & 0 & 0 & 0 & 0 \\
\hline $\begin{array}{l}\text { SYMPTOMS, SIGNS, \& ILL-DEFINED } \\
\text { CONDITIONS }\end{array}$ & $780-799$ & 19 & 54 & 1,282 & 1,248 & 2,603 \\
\hline INJURY \& POISONING & $800-999$ & 26 & 72 & 1,129 & 1,186 & 2,413 \\
\hline
\end{tabular}

\footnotetext{
*Absences with >1 ICD-9-CM code in the same diagnostic category were counted only once. Only those diagnostic categories and gender/age combinations with at least one occurrence appear in this table.
} 


\section{Savannah River Site 2008}

Absence Data

Appendix H. Total Number of Calendar Days Absent in Each Diagnostic Category by Gender and Age*

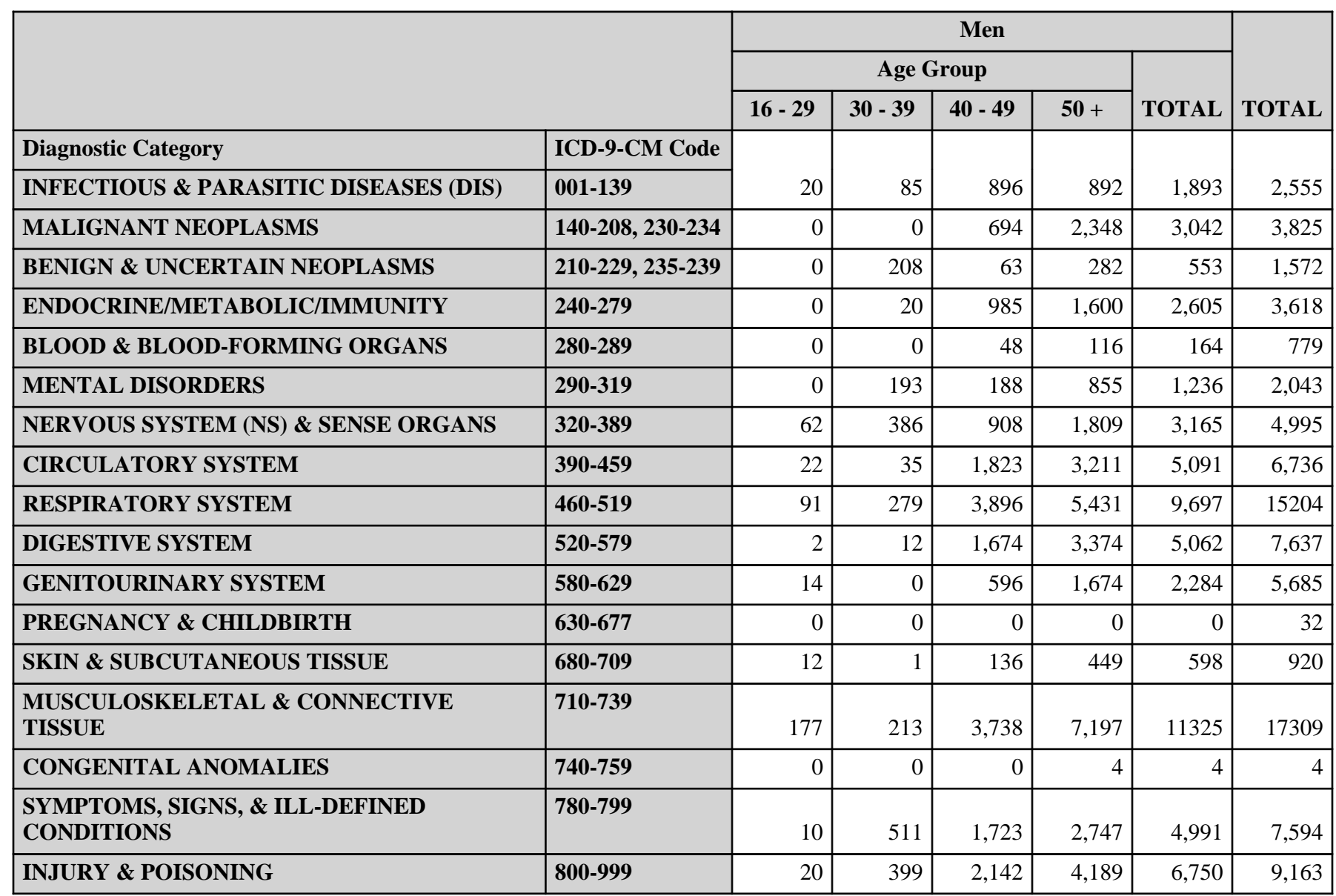

\footnotetext{
*Absences with >1 ICD-9-CM code in the same diagnostic category were counted only once. Only those diagnostic categories and gender/age combinations with at least one occurrence appear in this table.
} 


\section{Savannah River Site 2008}

\section{Absence Data}

Appendix I. Number of Diagnoses in Each Diagnostic Category by Gender and Job Category*

\begin{tabular}{|c|c|c|c|c|}
\hline & \\
\hline & & \multicolumn{3}{|c|}{ Job Category } \\
\hline & & Professional & $\begin{array}{c}\text { Administrative } \\
\text { Support }\end{array}$ & Technical Support \\
\hline Diagnostic Category & ICD-9-CM Code & \multirow[b]{2}{*}{16} & \multirow[b]{2}{*}{9} & \multirow[b]{2}{*}{22} \\
\hline INFECTIOUS \& PARASITIC DISEASES (DIS) & 001-139 & & & \\
\hline -Intestinal Infectious Dis & 001-009 & 2 & 5 & $\overline{9}$ \\
\hline -Other Bacterial Dis & 030-041 & 3 & 1 & 8 \\
\hline -Viral Dis with Exanthem & 050-057 & 1 & 1 & 2 \\
\hline -Other Viral Dis \& Chlamydiae & 070-079 & 3 & 2 & 3 \\
\hline -Mycoses & $110-118$ & 7 & 0 & 0 \\
\hline -Other Infections \& Parasitic Dis & $130-136$ & 0 & 0 & 0 \\
\hline MALIGNANT NEOPLASMS & 140-208, 230-234 & 13 & 4 & 9 \\
\hline -Digestive \& Peritoneal & $150-159$ & 4 & 0 & 0 \\
\hline -Bone, Connective Tissue, Skin & $170-173,176$ & 0 & 0 & 1 \\
\hline -Breast & $174-175$ & 1 & 1 & 8 \\
\hline -Genitourinary & 179-189 & 0 & 1 & 0 \\
\hline -Other \& Unspecified Sites & 190, 193-199 & 8 & 2 & 0 \\
\hline -Lymphatic \& Hematopoietic & $200-208$ & 0 & 0 & 0 \\
\hline BENIGN \& UNCERTAIN NEOPLASMS & 210-229, 235-239 & 9 & 6 & 9 \\
\hline ENDOCRINE/METABOLIC/IMMUNITY & $240-279$ & 7 & 6 & 20 \\
\hline -Thyroid Gland Disorders & $240-246$ & 0 & 1 & 2 \\
\hline -Other Endocrine Gland Dis & $250-259$ & 2 & 4 & 9 \\
\hline -Nutritional Deficiencies & $260-269$ & 0 & 0 & 2 \\
\hline -Other Metabolic \& Immunity Disorders & $270-279$ & 5 & 1 & 7 \\
\hline BLOOD \& BLOOD-FORMING ORGANS & $280-289$ & 12 & 2 & 3 \\
\hline MENTAL DISORDERS & $290-319$ & 2 & 16 & 10 \\
\hline -Psychoses & $290-299$ & 0 & 2 & 0 \\
\hline -Non-Psychotic Disorders & $300-302,306-316$ & 2 & 14 & 6 \\
\hline -Alcohol Dependence & 303 & 0 & 0 & 2 \\
\hline -Drug Dependence & $304-305$ & 0 & 0 & 2 \\
\hline NERVOUS SYSTEM (NS) \& SENSE ORGANS & 320-389 & 19 & 28 & 51 \\
\hline -Hereditary/Degenerative Central NS Dis & $330-337$ & 0 & 0 & 1 \\
\hline -Other Disorders of Central NS & $340-349$ & 5 & 1 & 13 \\
\hline -Disorders of Peripheral NS & $350-359$ & 2 & 2 & 12 \\
\hline -Disorders of Eye & $360-379$ & 7 & 17 & 16 \\
\hline -Dis of Ear \& Mastoid & $380-389$ & 5 & 8 & 9 \\
\hline CIRCULATORY SYSTEM & $390-459$ & 10 & 30 & 33 \\
\hline -Hypertensive Dis & $401-405$ & 2 & 11 & 27 \\
\hline -Ischemic Heart Dis & $410-414$ & 0 & 0 & 0 \\
\hline -Dis of Pulmonary Circulation & $415-417$ & 0 & 1 & 0 \\
\hline
\end{tabular}

(Continued)

*Only those diagnostic categories and gender/job category combinations with at least one occurrence appear in this table. 


\section{Savannah River Site 2008}

\section{Absence Data}

Appendix I. Number of Diagnoses in Each Diagnostic Category by Gender and Job Category*

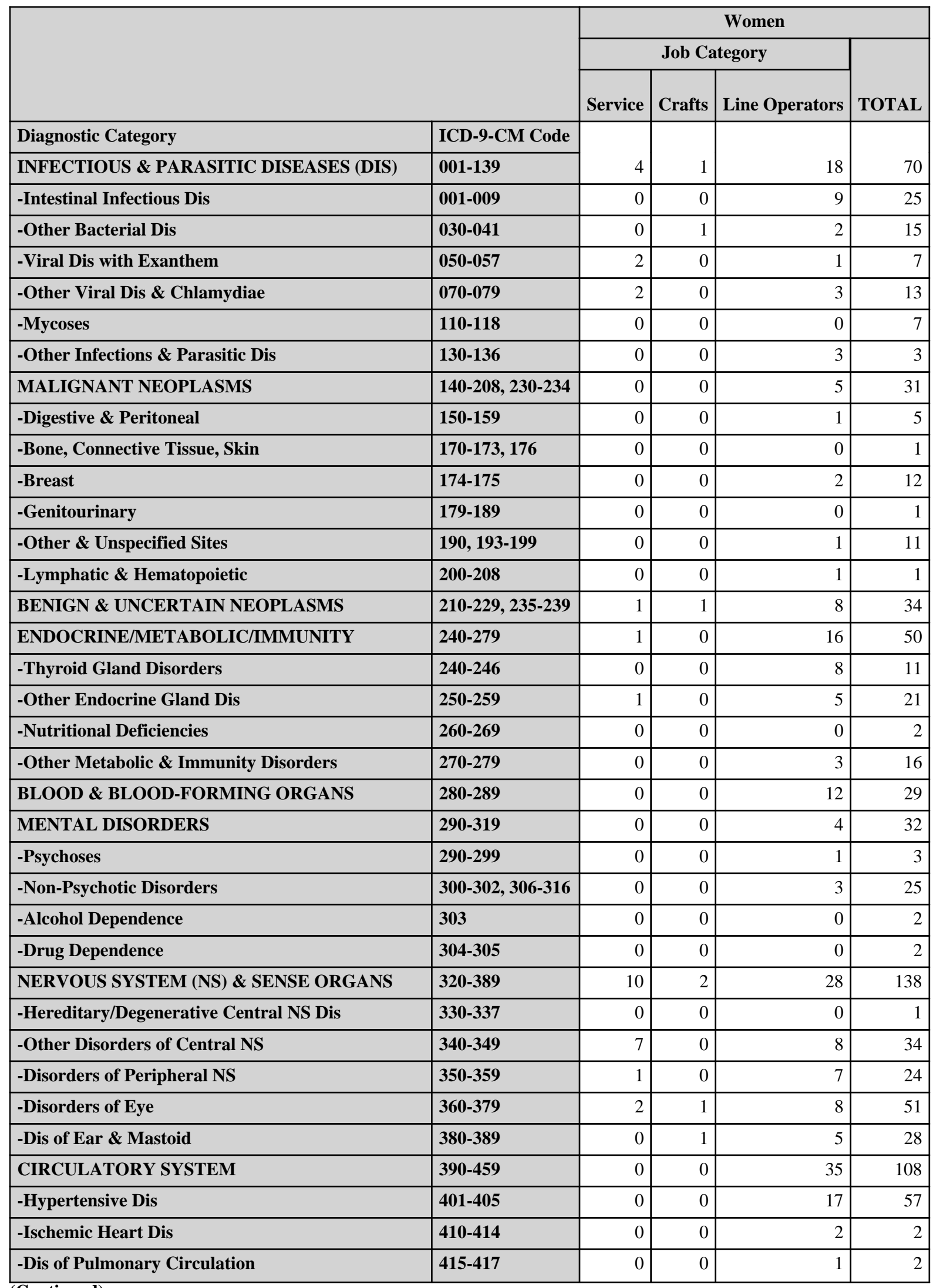

(Continued)

*Only those diagnostic categories and gender/job category combinations with at least one occurrence appear in this table. 


\section{Savannah River Site 2008}

\section{Absence Data}

Appendix I. Number of Diagnoses in Each Diagnostic Category by Gender and Job Category*

\begin{tabular}{|c|c|c|c|c|}
\hline & \multicolumn{3}{|c|}{ Women } \\
\hline & & \multicolumn{3}{|c|}{ Job Category } \\
\hline & & Professional & $\begin{array}{l}\text { Administrative } \\
\text { Support }\end{array}$ & Technical Support \\
\hline Diagnostic Category & ICD-9-CM Code & \multirow[b]{2}{*}{5} & \multirow[b]{2}{*}{12} & \multirow[b]{2}{*}{1} \\
\hline -Other Heart Dis & $420-429$ & & & \\
\hline -Cerebrovascular Dis & $430-438$ & 0 & 0 & 0 \\
\hline -Dis of Veins, Lymphatics, Other & 451-459 & 3 & 6 & 5 \\
\hline RESPIRATORY SYSTEM & $460-519$ & 93 & 109 & 170 \\
\hline -Acute Respiratory Infections & $460-466$ & 41 & 46 & 80 \\
\hline -Other Dis Upper Respiratory Tract & $470-478$ & 13 & 28 & 47 \\
\hline -Pneumonia \& Influenza & $480-487$ & 28 & 28 & 34 \\
\hline -Chronic Obstructive Dis & $490-496$ & 8 & 2 & $\overline{6}$ \\
\hline -Lung Dis from External Agents & $500-508$ & 3 & 0 & 0 \\
\hline -Other Respiratory Dis & $510-519$ & 0 & 5 & 3 \\
\hline DIGESTIVE SYSTEM & $520-579$ & 19 & 22 & 57 \\
\hline -Oral Cavity, Saliva Glands, Jaw & $520-529$ & 2 & 9 & 15 \\
\hline -Esophagus, Stomach, Duodenum & $530-537$ & 4 & 6 & $\overline{8}$ \\
\hline -Appendicitis & $540-543$ & 0 & 0 & 0 \\
\hline -Hernia & $550-553$ & 2 & 1 & 7 \\
\hline -Enteritis, Colitis & $555-558$ & 1 & 3 & 8 \\
\hline -Other Intestinal Dis & $560-569$ & 2 & 2 & 10 \\
\hline -Other Digestive Dis & $570-579$ & 8 & 1 & 9 \\
\hline GENITOURINARY SYSTEM & $580-629$ & 63 & 33 & 57 \\
\hline -Nephritis, Nephrosis & $580-589$ & 0 & 0 & 2 \\
\hline -Other Urinary Dis & $590-599$ & 24 & 3 & 21 \\
\hline -Breast Disorders & $610-611$ & 1 & 3 & 0 \\
\hline -Pelvic Inflammatory Dis & $614-616$ & 7 & 3 & 4 \\
\hline -Other Female Disorders & 617-629 & 31 & 24 & 30 \\
\hline PREGNANCY \& CHILDBIRTH & $630-677$ & 0 & 0 & 0 \\
\hline -Pregnancy with Abortive Outcome & $634-639$ & 0 & 0 & 0 \\
\hline SKIN \& SUBCUTANEOUS TISSUE & $680-709$ & 3 & 10 & 6 \\
\hline -Infections & $680-686$ & 2 & 3 & 4 \\
\hline -Other & 700-709 & 1 & 7 & 2 \\
\hline $\begin{array}{l}\text { MUSCULOSKELETAL \& CONNECTIVE } \\
\text { TISSUE }\end{array}$ & $710-739$ & 103 & 88 & 129 \\
\hline -Arthropathies & $710-719$ & 10 & 14 & 29 \\
\hline -Dorsopathies & $720-724$ & 69 & 51 & 75 \\
\hline -Rheumatism, Excluding Back & 725-729 & 19 & 19 & 21 \\
\hline -Other Dis \& Acquired Deformities & $730-739$ & 5 & 4 & 4 \\
\hline $\begin{array}{l}\text { SYMPTOMS, SIGNS, \& ILL-DEFINED } \\
\text { CONDITIONS }\end{array}$ & \begin{tabular}{|l|}
$780-799$ \\
\end{tabular} & 27 & 54 & 71 \\
\hline
\end{tabular}

(Continued)

*Only those diagnostic categories and gender/job category combinations with at least one occurrence appear in this table. 


\section{Savannah River Site 2008}

\section{Absence Data}

Appendix I. Number of Diagnoses in Each Diagnostic Category by Gender and Job Category*

\begin{tabular}{|c|c|c|c|c|c|}
\hline & & \multicolumn{4}{|c|}{ Women } \\
\hline & & \multicolumn{3}{|c|}{ Job Category } & \multirow[b]{2}{*}{ TOTAL } \\
\hline & & Service & Crafts & Line Operators & \\
\hline Diagnostic Category & ICD-9-CM Code & \multirow[b]{2}{*}{0} & \multirow[b]{2}{*}{0} & \multirow[b]{2}{*}{0} & \multirow[b]{2}{*}{18} \\
\hline -Other Heart Dis & 420-429 & & & & \\
\hline -Cerebrovascular Dis & $430-438$ & 0 & 0 & 2 & 2 \\
\hline -Dis of Veins, Lymphatics, Other & $451-459$ & 0 & 0 & 13 & 27 \\
\hline RESPIRATORY SYSTEM & $460-519$ & 18 & 1 & 139 & 530 \\
\hline -Acute Respiratory Infections & $460-466$ & 6 & 1 & 73 & 247 \\
\hline -Other Dis Upper Respiratory Tract & $470-478$ & 10 & 0 & 42 & 140 \\
\hline -Pneumonia \& Influenza & $480-487$ & 2 & 0 & 16 & 108 \\
\hline -Chronic Obstructive Dis & $490-496$ & 0 & 0 & 3 & 19 \\
\hline -Lung Dis from External Agents & $500-508$ & 0 & 0 & 0 & 3 \\
\hline -Other Respiratory Dis & $510-519$ & 0 & 0 & 5 & 13 \\
\hline DIGESTIVE SYSTEM & $520-579$ & 10 & 7 & 38 & 153 \\
\hline -Oral Cavity, Saliva Glands, Jaw & $520-529$ & 0 & 2 & 10 & 38 \\
\hline -Esophagus, Stomach, Duodenum & $530-537$ & 0 & 0 & 5 & 23 \\
\hline -Appendicitis & $540-543$ & 1 & 0 & 0 & 1 \\
\hline -Hernia & $550-553$ & 3 & 0 & 0 & 13 \\
\hline -Enteritis, Colitis & $555-558$ & 0 & 0 & 2 & 14 \\
\hline -Other Intestinal Dis & \begin{tabular}{|l|}
$560-569$ \\
\end{tabular} & 1 & 0 & 4 & 19 \\
\hline -Other Digestive Dis & $570-579$ & 5 & 5 & 17 & 45 \\
\hline GENITOURINARY SYSTEM & $580-629$ & 3 & 0 & 43 & 199 \\
\hline -Nephritis, Nephrosis & $580-589$ & 0 & 0 & 6 & 8 \\
\hline -Other Urinary Dis & $590-599$ & 0 & 0 & 13 & 61 \\
\hline -Breast Disorders & $610-611$ & 1 & 0 & 1 & 6 \\
\hline -Pelvic Inflammatory Dis & 614-616 & 0 & 0 & 2 & 16 \\
\hline -Other Female Disorders & $617-629$ & 2 & 0 & 21 & 108 \\
\hline PREGNANCY \& CHILDBIRTH & $630-677$ & 3 & 0 & 1 & 4 \\
\hline -Pregnancy with Abortive Outcome & $634-639$ & 3 & 0 & 1 & 4 \\
\hline SKIN \& SUBCUTANEOUS TISSUE & 680-709 & 2 & 1 & 1 & 23 \\
\hline -Infections & $680-686$ & 0 & 1 & 0 & 10 \\
\hline -Other & 700-709 & 2 & 0 & 1 & 13 \\
\hline $\begin{array}{l}\text { MUSCULOSKELETAL \& CONNECTIVE } \\
\text { TISSUE }\end{array}$ & 710-739 & 5 & 4 & 110 & 439 \\
\hline -Arthropathies & $710-719$ & 2 & 1 & 22 & 78 \\
\hline -Dorsopathies & $720-724$ & 1 & 3 & 73 & 272 \\
\hline -Rheumatism, Excluding Back & 725-729 & 2 & 0 & 12 & 73 \\
\hline -Other Dis \& Acquired Deformities & 730-739 & 0 & 0 & 3 & 16 \\
\hline $\begin{array}{l}\text { SYMPTOMS, SIGNS, \& ILL-DEFINED } \\
\text { CONDITIONS }\end{array}$ & 780-799 & 5 & 6 & 79 & 242 \\
\hline
\end{tabular}

(Continued)

*Only those diagnostic categories and gender/job category combinations with at least one occurrence appear in this table. 


\section{Savannah River Site 2008}

Absence Data

Appendix I. Number of Diagnoses in Each Diagnostic Category by Gender and Job Category*

\begin{tabular}{|c|c|c|c|c|}
\hline & & \multicolumn{3}{|c|}{ Women } \\
\hline & & \multicolumn{3}{|c|}{ Job Category } \\
\hline & & Professional & $\begin{array}{l}\text { Administrative } \\
\text { Support }\end{array}$ & Technical Support \\
\hline Diagnostic Category & ICD-9-CM Code & \multirow[b]{2}{*}{24} & \multirow[b]{2}{*}{53} & \multirow[b]{2}{*}{71} \\
\hline -Symptoms & 780-789 & & & \\
\hline -Non-Specific Abnormal Findings & $790-796$ & 1 & 1 & 0 \\
\hline -Ill-Defined \& Unknown Causes & 797-799 & 2 & 0 & 0 \\
\hline INJURY \& POISONING & $800-999$ & 29 & 19 & 58 \\
\hline -Fracture - Neck, Trunk & 805-809 & 6 & 0 & 1 \\
\hline -Fracture - Upper Limb & $810-819$ & 2 & 3 & 1 \\
\hline -Fracture - Lower Limb & $820-829$ & 1 & 7 & 4 \\
\hline -Dislocation & $830-839$ & 1 & 0 & 2 \\
\hline -Sprains \& Strains - Back & 846-847 & 2 & 0 & 15 \\
\hline -Sprains \& Strains - Other & $840-845,848$ & 3 & 1 & 9 \\
\hline -Intracranial Injury & $850-854$ & 1 & 0 & 1 \\
\hline -Open Wound - Head, Neck, Trunk & $870-879$ & 2 & 0 & 5 \\
\hline -Open Wound - Lower Limb & 890-897 & 0 & 0 & 1 \\
\hline -Superficial Injury & $910-919$ & 2 & 0 & 2 \\
\hline -Contusion & 920-924 & 3 & 0 & 11 \\
\hline -Unspecified Effects - External Causes & $990-995$ & 1 & 4 & 3 \\
\hline -Complications of Surgical/Medical Care & 996-999 & 5 & 4 & 3 \\
\hline $\begin{array}{l}\text { HEALTH STATUS/HEALTH SERVICE } \\
\text { CONTACT }\end{array}$ & V01-V82 & 14 & 14 & 38 \\
\hline -Isolation \& Prophylactic Measures & V07-V09 & 1 & 1 & 0 \\
\hline -Personal \& Family History & V10-V19 & 2 & 3 & 1 \\
\hline -Health Services Reproduction/Development & V20-V29 & 4 & 3 & 8 \\
\hline -Health Status & V40-V49 & 0 & 2 & 10 \\
\hline -Specific Procedures/Aftercare & V50-V59 & 5 & 5 & 18 \\
\hline -Examination \& Investigation & V70-V82 & 2 & 0 & 1 \\
\hline
\end{tabular}

*Only those diagnostic categories and gender/job category combinations with at least one occurrence appear in this table. 


\section{Savannah River Site 2008}

Absence Data

Appendix I. Number of Diagnoses in Each Diagnostic Category by Gender and Job Category*

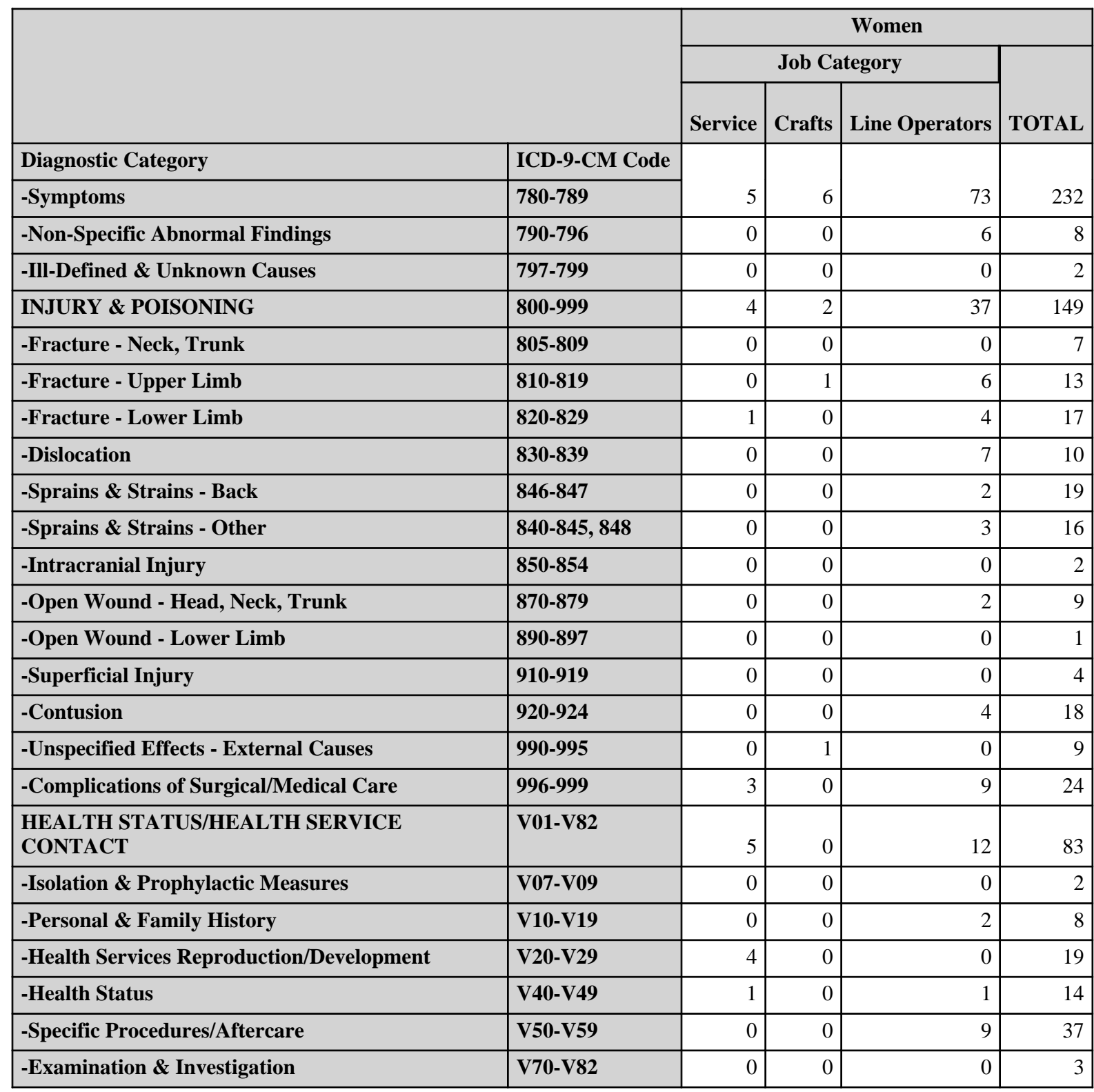

\begin{tabular}{|c|c|c|c|c|c|c|c|}
\hline & \multicolumn{7}{|c|}{ Women } \\
\hline & \multicolumn{6}{|c|}{ Job Category } & \multirow[b]{2}{*}{ TOTAL } \\
\hline & Professional & \begin{tabular}{|c} 
Administrative \\
Support
\end{tabular} & Technical Support & Service & Crafts & Line Operators & \\
\hline Diagnostic Category & \multirow[b]{2}{*}{439} & \multirow[b]{2}{*}{450} & \multirow[b]{2}{*}{743} & \multirow[b]{2}{*}{71} & \multirow[b]{2}{*}{25} & \multirow[b]{2}{*}{586} & \multirow[b]{2}{*}{2,314} \\
\hline Total & & & & & & & \\
\hline
\end{tabular}

*Only those diagnostic categories and gender/job category combinations with at least one occurrence appear in this table. 


\section{Savannah River Site 2008}

\section{Absence Data}

Appendix I. Number of Diagnoses in Each Diagnostic Category by Gender and Job Category*

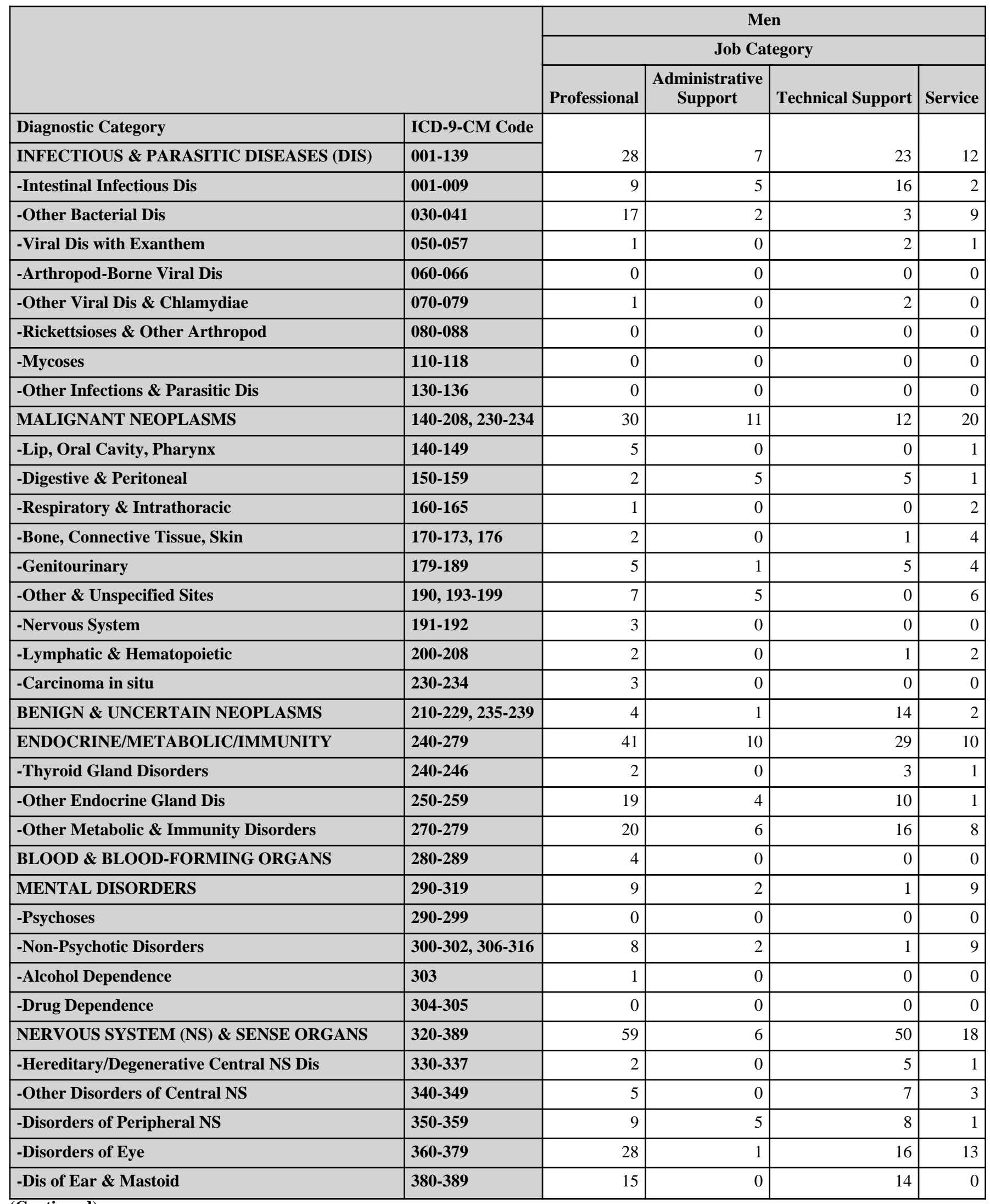

(Continued)

*Only those diagnostic categories and gender/job category combinations with at least one occurrence appear in this table. 


\section{Savannah River Site 2008}

\section{Absence Data}

Appendix I. Number of Diagnoses in Each Diagnostic Category by Gender and Job Category*

\begin{tabular}{|c|c|c|c|c|c|}
\hline & & \multicolumn{4}{|c|}{ Men } \\
\hline & & \multicolumn{3}{|c|}{ Job Category } & \multirow[b]{2}{*}{ TOTAL } \\
\hline & & Security and Fire & Crafts & Line Operators & \\
\hline Diagnostic Category & ICD-9-CM Code & \multirow[b]{2}{*}{1} & \multirow[b]{2}{*}{1} & \multirow[b]{2}{*}{73} & \multirow[b]{2}{*}{145} \\
\hline INFECTIOUS \& PARASITIC DISEASES (DIS) & 001-139 & & & & \\
\hline -Intestinal Infectious Dis & 001-009 & 0 & 0 & 40 & 72 \\
\hline -Other Bacterial Dis & 030-041 & 0 & 1 & 17 & 49 \\
\hline -Viral Dis with Exanthem & 050-057 & 0 & 0 & 3 & 7 \\
\hline -Arthropod-Borne Viral Dis & $060-066$ & 1 & 0 & 0 & 1 \\
\hline -Other Viral Dis \& Chlamydiae & 070-079 & 0 & 0 & 5 & 8 \\
\hline -Rickettsioses \& Other Arthropod & 080-088 & 0 & 0 & 3 & 3 \\
\hline -Mycoses & 110-118 & 0 & 0 & 4 & 4 \\
\hline -Other Infections \& Parasitic Dis & $130-136$ & 0 & 0 & 1 & 1 \\
\hline MALIGNANT NEOPLASMS & 140-208, 230-234 & 0 & 0 & 24 & 97 \\
\hline -Lip, Oral Cavity, Pharynx & $140-149$ & 0 & 0 & 0 & 6 \\
\hline -Digestive \& Peritoneal & 150-159 & 0 & 0 & 1 & 14 \\
\hline -Respiratory \& Intrathoracic & $160-165$ & 0 & 0 & 2 & 5 \\
\hline -Bone, Connective Tissue, Skin & $170-173,176$ & 0 & 0 & 8 & 15 \\
\hline -Genitourinary & 179-189 & 0 & 0 & 11 & 26 \\
\hline -Other \& Unspecified Sites & $190,193-199$ & 0 & 0 & 2 & 20 \\
\hline -Nervous System & 191-192 & 0 & 0 & 0 & 3 \\
\hline -Lymphatic \& Hematopoietic & $200-208$ & 0 & 0 & 0 & 5 \\
\hline -Carcinoma in situ & $230-234$ & 0 & 0 & 0 & 3 \\
\hline BENIGN \& UNCERTAIN NEOPLASMS & 210-229, 235-239 & 1 & 0 & 8 & 30 \\
\hline ENDOCRINE/METABOLIC/IMMUNITY & $240-279$ & 0 & 4 & 34 & 128 \\
\hline -Thyroid Gland Disorders & $240-246$ & 0 & 1 & 2 & 9 \\
\hline -Other Endocrine Gland Dis & $250-259$ & 0 & 2 & 12 & 48 \\
\hline -Other Metabolic \& Immunity Disorders & $270-279$ & 0 & 1 & 20 & 71 \\
\hline BLOOD \& BLOOD-FORMING ORGANS & $280-289$ & 0 & 0 & 4 & 8 \\
\hline MENTAL DISORDERS & $290-319$ & 0 & 2 & 26 & 49 \\
\hline -Psychoses & 290-299 & 0 & 0 & 2 & 2 \\
\hline -Non-Psychotic Disorders & $300-302,306-316$ & 0 & 2 & 21 & 43 \\
\hline -Alcohol Dependence & 303 & 0 & 0 & 2 & 3 \\
\hline -Drug Dependence & 304-305 & 0 & 0 & 1 & 1 \\
\hline NERVOUS SYSTEM (NS) \& SENSE ORGANS & $320-389$ & 0 & 3 & 70 & 206 \\
\hline -Hereditary/Degenerative Central NS Dis & 330-337 & 0 & 0 & 0 & 8 \\
\hline -Other Disorders of Central NS & $340-349$ & 0 & 0 & 7 & 22 \\
\hline -Disorders of Peripheral NS & $350-359$ & 0 & 0 & 8 & 31 \\
\hline -Disorders of Eye & $360-379$ & 0 & 2 & 27 & 87 \\
\hline -Dis of Ear \& Mastoid & $380-389$ & 0 & 1 & 24 & 54 \\
\hline
\end{tabular}

(Continued)

*Only those diagnostic categories and gender/job category combinations with at least one occurrence appear in this table. 


\section{Savannah River Site 2008}

\section{Absence Data}

Appendix I. Number of Diagnoses in Each Diagnostic Category by Gender and Job Category*

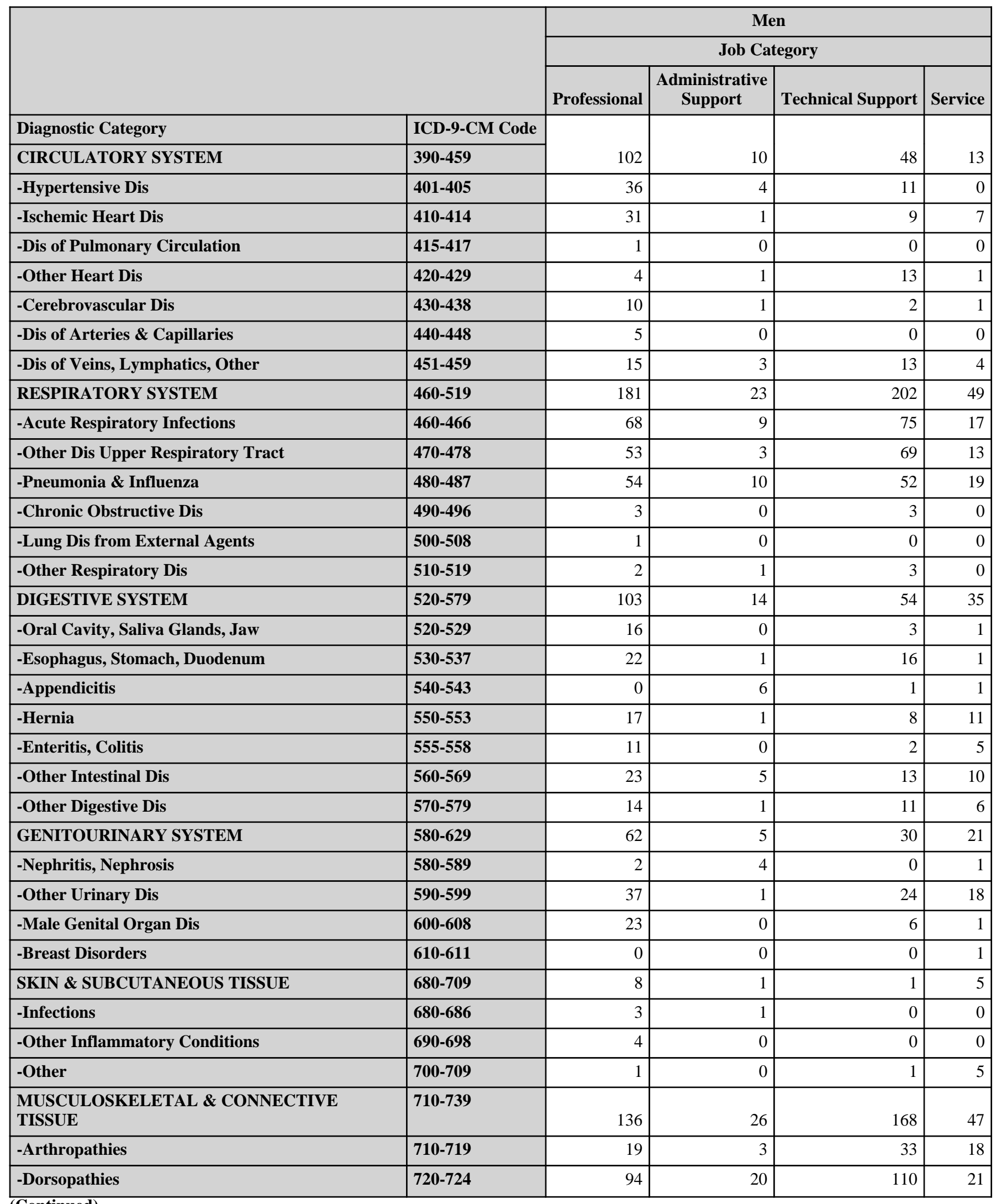

(Continued)

*Only those diagnostic categories and gender/job category combinations with at least one occurrence appear in this table. 


\section{Savannah River Site 2008}

\section{Absence Data}

Appendix I. Number of Diagnoses in Each Diagnostic Category by Gender and Job Category*

\begin{tabular}{|c|c|c|c|c|c|}
\hline & & \multicolumn{4}{|c|}{ Men } \\
\hline & & \multicolumn{3}{|c|}{ Job Category } & \multirow[b]{2}{*}{ TOTAL } \\
\hline & & Security and Fire & Crafts & Line Operators & \\
\hline Diagnostic Category & ICD-9-CM Code & \multirow[b]{2}{*}{1} & \multirow[b]{2}{*}{8} & \multirow[b]{2}{*}{95} & \multirow[b]{2}{*}{277} \\
\hline CIRCULATORY SYSTEM & $390-459$ & & & & \\
\hline -Hypertensive Dis & $401-405$ & 0 & 4 & 42 & 97 \\
\hline -Ischemic Heart Dis & $410-414$ & 1 & 1 & 14 & 64 \\
\hline -Dis of Pulmonary Circulation & $415-417$ & 0 & 0 & 0 & 1 \\
\hline -Other Heart Dis & $420-429$ & 0 & 2 & 15 & 36 \\
\hline -Cerebrovascular Dis & $430-438$ & 0 & 1 & 3 & 18 \\
\hline -Dis of Arteries \& Capillaries & $440-448$ & 0 & 0 & 2 & 7 \\
\hline -Dis of Veins, Lymphatics, Other & 451-459 & 0 & 0 & 19 & 54 \\
\hline RESPIRATORY SYSTEM & $460-519$ & 1 & 9 & 441 & 906 \\
\hline -Acute Respiratory Infections & $460-466$ & 0 & 3 & 196 & 368 \\
\hline -Other Dis Upper Respiratory Tract & $470-478$ & 1 & 2 & 116 & 257 \\
\hline -Pneumonia \& Influenza & $480-487$ & 0 & 4 & 112 & 251 \\
\hline -Chronic Obstructive Dis & $490-496$ & 0 & 0 & 8 & 14 \\
\hline -Lung Dis from External Agents & $500-508$ & 0 & 0 & 6 & 7 \\
\hline -Other Respiratory Dis & $510-519$ & 0 & 0 & 3 & 9 \\
\hline DIGESTIVE SYSTEM & $520-579$ & 1 & 4 & 121 & 332 \\
\hline -Oral Cavity, Saliva Glands, Jaw & $520-529$ & 1 & 2 & 14 & 37 \\
\hline -Esophagus, Stomach, Duodenum & $530-537$ & 0 & 1 & 32 & 73 \\
\hline -Appendicitis & $540-543$ & 0 & 0 & 0 & 8 \\
\hline -Hernia & $550-553$ & 0 & 0 & 23 & 60 \\
\hline -Enteritis, Colitis & $555-558$ & 0 & 1 & 7 & 26 \\
\hline -Other Intestinal Dis & $560-569$ & 0 & 0 & 36 & 87 \\
\hline -Other Digestive Dis & $570-579$ & 0 & 0 & 9 & 41 \\
\hline GENITOURINARY SYSTEM & $580-629$ & 0 & 3 & 66 & 187 \\
\hline -Nephritis, Nephrosis & $580-589$ & 0 & 0 & 1 & 8 \\
\hline -Other Urinary Dis & 590-599 & 0 & 3 & 52 & 135 \\
\hline -Male Genital Organ Dis & $600-608$ & 0 & 0 & 12 & 42 \\
\hline -Breast Disorders & $610-611$ & 0 & 0 & 1 & 2 \\
\hline SKIN \& SUBCUTANEOUS TISSUE & 680-709 & 0 & 4 & 24 & 43 \\
\hline -Infections & $680-686$ & 0 & 1 & 5 & 10 \\
\hline -Other Inflammatory Conditions & $690-698$ & 0 & 0 & 7 & 11 \\
\hline -Other & 700-709 & 0 & 3 & 12 & 22 \\
\hline $\begin{array}{l}\text { MUSCULOSKELETAL \& CONNECTIVE } \\
\text { TISSUE }\end{array}$ & 710-739 & 0 & 19 & 247 & 643 \\
\hline -Arthropathies & $710-719$ & 0 & 6 & 80 & 159 \\
\hline -Dorsopathies & $720-724$ & 0 & 9 & 136 & 390 \\
\hline
\end{tabular}

(Continued)

*Only those diagnostic categories and gender/job category combinations with at least one occurrence appear in this table. 


\section{Savannah River Site 2008}

\section{Absence Data}

Appendix I. Number of Diagnoses in Each Diagnostic Category by Gender and Job Category*

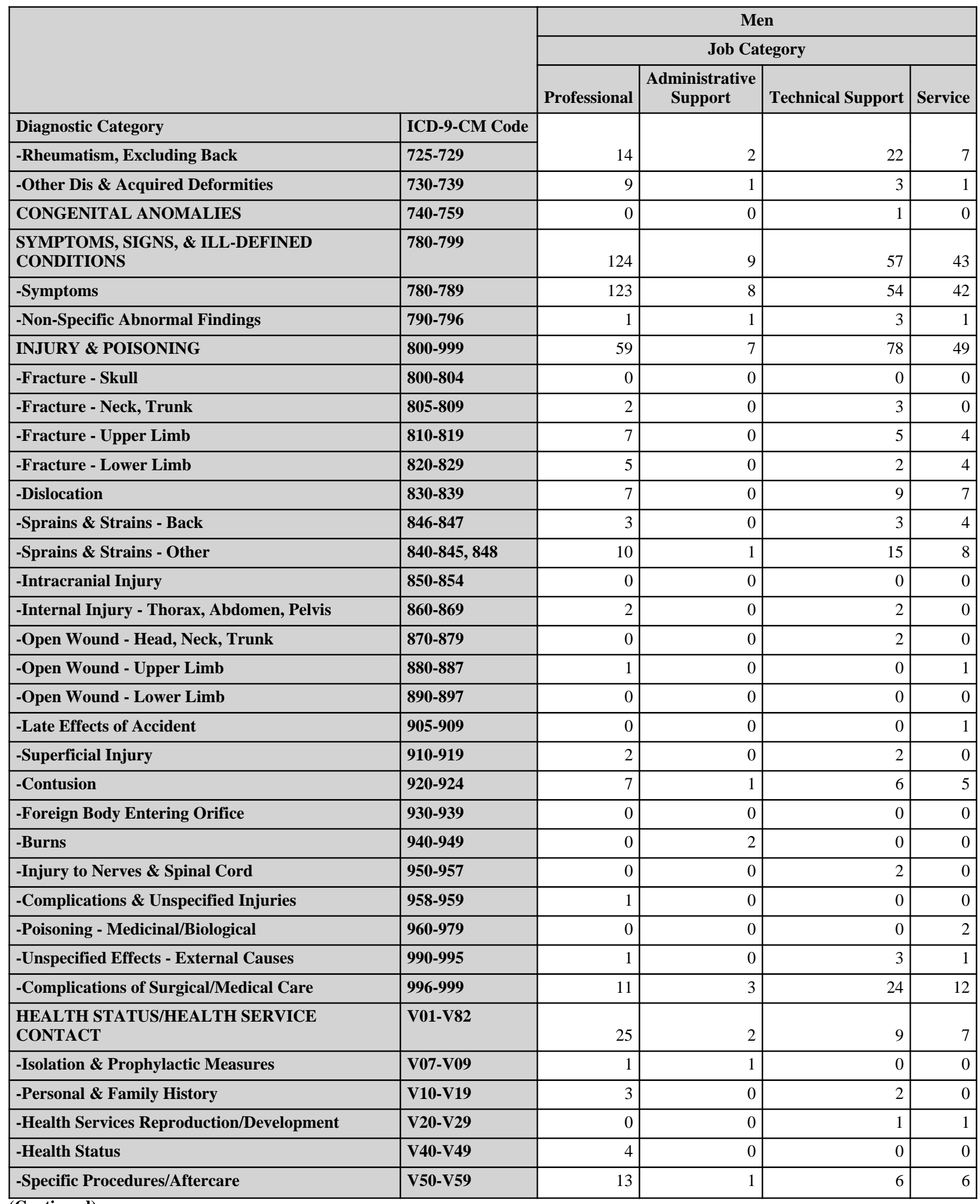

(Continued)

*Only those diagnostic categories and gender/job category combinations with at least one occurrence appear in this table. 


\section{Savannah River Site 2008}

\section{Absence Data}

Appendix I. Number of Diagnoses in Each Diagnostic Category by Gender and Job Category*

\begin{tabular}{|c|c|c|c|c|c|}
\hline & & \multicolumn{4}{|c|}{ Men } \\
\hline & & \multicolumn{3}{|c|}{ Job Category } & \multirow[b]{2}{*}{ TOTAL } \\
\hline & & Security and Fire & Crafts & Line Operators & \\
\hline Diagnostic Category & ICD-9-CM Code & \multirow[b]{2}{*}{0} & \multirow[b]{2}{*}{2} & \multirow[b]{2}{*}{29} & \multirow[b]{2}{*}{76} \\
\hline -Rheumatism, Excluding Back & $725-729$ & & & & \\
\hline -Other Dis \& Acquired Deformities & $730-739$ & 0 & 2 & 2 & 18 \\
\hline CONGENITAL ANOMALIES & $740-759$ & 0 & 0 & 0 & 1 \\
\hline $\begin{array}{l}\text { SYMPTOMS, SIGNS, \& ILL-DEFINED } \\
\text { CONDITIONS }\end{array}$ & $780-799$ & 1 & 9 & 197 & 440 \\
\hline -Symptoms & $780-789$ & 1 & 9 & 194 & 431 \\
\hline -Non-Specific Abnormal Findings & $790-796$ & 0 & 0 & 3 & 9 \\
\hline INJURY \& POISONING & $800-999$ & 2 & 6 & 151 & 352 \\
\hline -Fracture - Skull & $800-804$ & 0 & 0 & 2 & 2 \\
\hline -Fracture - Neck, Trunk & 805-809 & 0 & 0 & 5 & 10 \\
\hline -Fracture - Upper Limb & $810-819$ & 0 & 0 & 4 & 20 \\
\hline -Fracture - Lower Limb & $820-829$ & 0 & 1 & 3 & 15 \\
\hline -Dislocation & 830-839 & 2 & 1 & 19 & 45 \\
\hline -Sprains \& Strains - Back & 846-847 & 0 & 2 & 28 & 40 \\
\hline -Sprains \& Strains - Other & $840-845,848$ & 0 & 0 & 31 & 65 \\
\hline -Intracranial Injury & $850-854$ & 0 & 0 & 5 & 5 \\
\hline -Internal Injury - Thorax, Abdomen, Pelvis & $860-869$ & 0 & 0 & 3 & 7 \\
\hline -Open Wound - Head, Neck, Trunk & $870-879$ & 0 & 0 & 2 & 4 \\
\hline -Open Wound - Upper Limb & 880-887 & 0 & 1 & 2 & 5 \\
\hline -Open Wound - Lower Limb & 890-897 & 0 & 0 & 7 & 7 \\
\hline -Late Effects of Accident & 905-909 & 0 & 0 & 0 & 1 \\
\hline -Superficial Injury & 910-919 & 0 & 0 & 6 & 10 \\
\hline -Contusion & $920-924$ & 0 & 1 & 12 & 32 \\
\hline -Foreign Body Entering Orifice & 930-939 & 0 & 0 & 2 & 2 \\
\hline -Burns & $940-949$ & 0 & 0 & 4 & 6 \\
\hline -Injury to Nerves \& Spinal Cord & 950-957 & 0 & 0 & 0 & 2 \\
\hline -Complications \& Unspecified Injuries & 958-959 & 0 & 0 & 0 & 1 \\
\hline -Poisoning - Medicinal/Biological & $960-979$ & 0 & 0 & 0 & 2 \\
\hline -Unspecified Effects - External Causes & $990-995$ & 0 & 0 & 5 & 10 \\
\hline -Complications of Surgical/Medical Care & 996-999 & 0 & 0 & 11 & 61 \\
\hline $\begin{array}{l}\text { HEALTH STATUS/HEALTH SERVICE } \\
\text { CONTACT }\end{array}$ & V01-V82 & 0 & 0 & 25 & 68 \\
\hline -Isolation \& Prophylactic Measures & V07-V09 & 0 & 0 & 1 & 3 \\
\hline -Personal \& Family History & V10-V19 & 0 & 0 & 6 & 11 \\
\hline -Health Services Reproduction/Development & V20-V29 & 0 & 0 & 1 & 3 \\
\hline -Health Status & V40-V49 & 0 & 0 & 2 & 6 \\
\hline -Specific Procedures/Aftercare & V50-V59 & 0 & 0 & 8 & 34 \\
\hline
\end{tabular}

(Continued)

*Only those diagnostic categories and gender/job category combinations with at least one occurrence appear in this table. 


\section{Savannah River Site 2008}

Absence Data

Appendix I. Number of Diagnoses in Each Diagnostic Category by Gender and Job Category*

\begin{tabular}{|c|c|c|c|c|c|}
\hline & & \multicolumn{4}{|c|}{ Men } \\
\hline & & \multicolumn{4}{|c|}{ Job Category } \\
\hline & & Professional & $\begin{array}{c}\text { Administrative } \\
\text { Support }\end{array}$ & Technical Support & Service \\
\hline Diagnostic Category & ICD-9-CM Code & \multirow[b]{2}{*}{0} & \multirow[b]{2}{*}{0} & \multirow[b]{2}{*}{0} & \multirow[b]{2}{*}{0} \\
\hline -Other Circumstances & $\begin{array}{l}\text { V60-V69 } \\
\end{array}$ & & & & \\
\hline
\end{tabular}

*Only those diagnostic categories and gender/job category combinations with at least one occurrence appear in this table. 


\section{Savannah River Site 2008}

\section{Absence Data}

Appendix I. Number of Diagnoses in Each Diagnostic Category by Gender and Job Category*

\begin{tabular}{|c|c|c|c|c|c|}
\hline & & \multicolumn{4}{|c|}{ Men } \\
\hline & & \multicolumn{3}{|c|}{ Job Category } & \multirow[b]{2}{*}{ TOTAL } \\
\hline & & Security and Fire & Crafts & Line Operators & \\
\hline Diagnostic Category & ICD-9-CM Code & \multirow[b]{2}{*}{0} & \multirow[b]{2}{*}{0} & \multirow[b]{2}{*}{6} & \multirow[b]{2}{*}{6} \\
\hline -Other Circumstances & $\begin{array}{l}\text { V60-V69 } \\
\end{array}$ & & & & \\
\hline -Examination \& Investigation & V70-V82 & 0 & 0 & 1 & 5 \\
\hline
\end{tabular}

\begin{tabular}{|c|c|c|c|c|c|c|c|c|}
\hline & \multicolumn{8}{|c|}{ Men } \\
\hline & \multicolumn{7}{|c|}{ Job Category } & \multirow[b]{2}{*}{ TOTAL } \\
\hline & Professional & $\begin{array}{c}\text { Administrative } \\
\text { Support }\end{array}$ & $\begin{array}{l}\text { Technical } \\
\text { Support }\end{array}$ & Service & $\begin{array}{c}\text { Security and } \\
\text { Fire }\end{array}$ & Crafts & $\begin{array}{c}\text { Line } \\
\text { Operators }\end{array}$ & \\
\hline Diagnostic Category & \multirow[b]{2}{*}{975} & \multirow[b]{2}{*}{134} & \multirow[b]{2}{*}{777} & \multirow[b]{2}{*}{340} & \multirow[b]{2}{*}{8} & \multirow[b]{2}{*}{72} & \multirow[b]{2}{*}{1,606} & \multirow[b]{2}{*}{3,912} \\
\hline Total & & & & & & & & \\
\hline
\end{tabular}

*Only those diagnostic categories and gender/job category combinations with at least one occurrence appear in this table. 


\section{Savannah River Site 2008}

\section{Absence Data}

Appendix J. Total Number of Calendar Days Absent in Each Diagnostic Category by Gender and Job Category*

\begin{tabular}{|c|c|c|c|c|}
\hline & & \multicolumn{3}{|c|}{ Women } \\
\hline & & \multicolumn{3}{|c|}{ Job Category } \\
\hline & & Professional & $\begin{array}{l}\text { Administrative } \\
\text { Support }\end{array}$ & Technical Support \\
\hline Diagnostic Category & ICD-9-CM Code & \multirow[b]{2}{*}{150} & \multirow[b]{2}{*}{86} & \multirow[b]{2}{*}{188} \\
\hline INFECTIOUS \& PARASITIC DISEASES (DIS) & 001-139 & & & \\
\hline MALIGNANT NEOPLASMS & $140-208,230-234$ & 93 & 71 & 131 \\
\hline BENIGN \& UNCERTAIN NEOPLASMS & 210-229, 235-239 & 188 & 251 & 261 \\
\hline ENDOCRINE/METABOLIC/IMMUNITY & $240-279$ & 149 & 161 & 268 \\
\hline BLOOD \& BLOOD-FORMING ORGANS & $280-289$ & 141 & 133 & 94 \\
\hline MENTAL DISORDERS & 290-319 & 17 & 590 & 106 \\
\hline NERVOUS SYSTEM (NS) \& SENSE ORGANS & $320-389$ & 385 & 310 & 528 \\
\hline CIRCULATORY SYSTEM & $390-459$ & 257 & 684 & 371 \\
\hline RESPIRATORY SYSTEM & $460-519$ & 1,269 & 1,238 & 1,588 \\
\hline DIGESTIVE SYSTEM & $520-579$ & 417 & 291 & 482 \\
\hline GENITOURINARY SYSTEM & $580-629$ & 830 & 622 & 976 \\
\hline PREGNANCY \& CHILDBIRTH & $630-677$ & 0 & 0 & 0 \\
\hline SKIN \& SUBCUTANEOUS TISSUE & 680-709 & 28 & 173 & 45 \\
\hline $\begin{array}{l}\text { MUSCULOSKELETAL \& CONNECTIVE } \\
\text { TISSUE }\end{array}$ & $710-739$ & 1,280 & 1,199 & 1,848 \\
\hline $\begin{array}{l}\text { SYMPTOMS, SIGNS, \& ILL-DEFINED } \\
\text { CONDITIONS }\end{array}$ & $780-799$ & 472 & 656 & 565 \\
\hline INJURY \& POISONING & $800-999$ & 580 & 530 & 444 \\
\hline
\end{tabular}

\footnotetext{
*Absences with >1 ICD-9-CM code in the same diagnostic category were counted only once. Only those diagnostic categories and gender/job category combinations with at least one occurrence appear in this table.
} 


\section{Savannah River Site 2008}

\section{Absence Data}

Appendix J. Total Number of Calendar Days Absent in Each Diagnostic Category by Gender and Job Category*

\begin{tabular}{|c|c|c|c|c|c|}
\hline & & \multicolumn{4}{|c|}{ Women } \\
\hline & & \multicolumn{3}{|c|}{ Job Category } & \multirow[b]{2}{*}{ TOTAL } \\
\hline & & Service & Crafts & Line Operators & \\
\hline Diagnostic Category & ICD-9-CM Code & \multirow[b]{2}{*}{32} & \multirow[b]{2}{*}{20} & \multirow[b]{2}{*}{186} & \multirow[b]{2}{*}{662} \\
\hline INFECTIOUS \& PARASITIC DISEASES (DIS) & 001-139 & & & & \\
\hline MALIGNANT NEOPLASMS & $140-208,230-234$ & 0 & 0 & 488 & 783 \\
\hline BENIGN \& UNCERTAIN NEOPLASMS & 210-229, 235-239 & 14 & 6 & 299 & 1,019 \\
\hline ENDOCRINE/METABOLIC/IMMUNITY & $240-279$ & 1 & 0 & 434 & 1,013 \\
\hline BLOOD \& BLOOD-FORMING ORGANS & $280-289$ & 0 & 0 & 247 & 615 \\
\hline MENTAL DISORDERS & 290-319 & 0 & 0 & 94 & 807 \\
\hline NERVOUS SYSTEM (NS) \& SENSE ORGANS & $320-389$ & 195 & 15 & 397 & 1,830 \\
\hline CIRCULATORY SYSTEM & $390-459$ & 0 & 0 & 333 & 1,645 \\
\hline RESPIRATORY SYSTEM & $460-519$ & 155 & 8 & 1,249 & 5,507 \\
\hline DIGESTIVE SYSTEM & $520-579$ & 284 & 97 & 1,004 & 2,575 \\
\hline GENITOURINARY SYSTEM & $580-629$ & 33 & 0 & 940 & 3,401 \\
\hline PREGNANCY \& CHILDBIRTH & $630-677$ & 25 & 0 & 7 & 32 \\
\hline SKIN \& SUBCUTANEOUS TISSUE & 680-709 & 30 & 18 & 28 & 322 \\
\hline $\begin{array}{l}\text { MUSCULOSKELETAL \& CONNECTIVE } \\
\text { TISSUE }\end{array}$ & $710-739$ & 56 & 28 & 1,573 & 5,984 \\
\hline $\begin{array}{l}\text { SYMPTOMS, SIGNS, \& ILL-DEFINED } \\
\text { CONDITIONS }\end{array}$ & $780-799$ & 33 & 86 & 791 & 2,603 \\
\hline INJURY \& POISONING & 800-999 & 48 & 25 & 786 & 2,413 \\
\hline
\end{tabular}

\footnotetext{
*Absences with >1 ICD-9-CM code in the same diagnostic category were counted only once. Only those diagnostic categories and gender/job category combinations with at least one occurrence appear in this table.
} 


\section{Savannah River Site 2008}

\section{Absence Data}

Appendix J. Total Number of Calendar Days Absent in Each Diagnostic Category by Gender and Job Category*

\begin{tabular}{|c|c|c|c|c|c|}
\hline & & \multicolumn{4}{|c|}{ Men } \\
\hline & & \multicolumn{4}{|c|}{ Job Category } \\
\hline & & Professional & $\begin{array}{l}\text { Administrative } \\
\text { Support }\end{array}$ & Technical Support & Service \\
\hline Diagnostic Category & ICD-9-CM Code & \multirow[b]{2}{*}{413} & \multirow[b]{2}{*}{186} & \multirow[b]{2}{*}{217} & \multirow[b]{2}{*}{84} \\
\hline INFECTIOUS \& PARASITIC DISEASES (DIS) & 001-139 & & & & \\
\hline BENIGN \& UNCERTAIN NEOPLASMS & 210-229, 235-239 & 122 & 1 & 272 & 15 \\
\hline ENDOCRINE/METABOLIC/IMMUNITY & $240-279$ & 897 & 404 & 485 & 156 \\
\hline BLOOD \& BLOOD-FORMING ORGANS & 280-289 & 117 & 0 & 0 & 0 \\
\hline MENTAL DISORDERS & 290-319 & 551 & 32 & 14 & 306 \\
\hline DIGESTIVE SYSTEM & $520-579$ & 1,671 & 349 & 851 & 464 \\
\hline GENITOURINARY SYSTEM & $580-629$ & 749 & 222 & 296 & 141 \\
\hline SKIN \& SUBCUTANEOUS TISSUE & 680-709 & 108 & 7 & 84 & 29 \\
\hline $\begin{array}{l}\text { MUSCULOSKELETAL \& CONNECTIVE } \\
\text { TISSUE }\end{array}$ & 710-739 & 2,549 & 706 & 2,333 & 859 \\
\hline CONGENITAL ANOMALIES & 740-759 & 0 & 0 & 4 & 0 \\
\hline $\begin{array}{l}\text { SYMPTOMS, SIGNS, \& ILL-DEFINED } \\
\text { CONDITIONS }\end{array}$ & 780-799 & 1,425 & 88 & 598 & 607 \\
\hline INJURY \& POISONING & $800-999$ & 1,491 & 210 & 1,697 & 820 \\
\hline
\end{tabular}

\footnotetext{
*Absences with >1 ICD-9-CM code in the same diagnostic category were counted only once. Only those diagnostic categories and gender/job category combinations with at least one occurrence appear in this table.
} 


\section{Savannah River Site 2008}

\section{Absence Data}

Appendix J. Total Number of Calendar Days Absent in Each Diagnostic Category by Gender and Job Category*

\begin{tabular}{|c|c|c|c|c|c|}
\hline & & \multicolumn{4}{|c|}{ Men } \\
\hline & & \multicolumn{3}{|c|}{ Job Category } & \multirow[b]{2}{*}{ TOTAL } \\
\hline & & Security and Fire & Crafts & Line Operators & \\
\hline Diagnostic Category & ICD-9-CM Code & \multirow[b]{2}{*}{8} & \multirow[b]{2}{*}{14} & \multirow[b]{2}{*}{971} & \multirow[b]{2}{*}{1,893} \\
\hline INFECTIOUS \& PARASITIC DISEASES (DIS) & 001-139 & & & & \\
\hline MALIGNANT NEOPLASMS & $140-208,230-234$ & 0 & 0 & 403 & 3,042 \\
\hline BENIGN \& UNCERTAIN NEOPLASMS & 210-229, 235-239 & 24 & 0 & 119 & 553 \\
\hline ENDOCRINE/METABOLIC/IMMUNITY & $240-279$ & 0 & 19 & 644 & 2,605 \\
\hline BLOOD \& BLOOD-FORMING ORGANS & 280-289 & 0 & 0 & 47 & 164 \\
\hline MENTAL DISORDERS & 290-319 & 0 & 13 & 320 & 1,236 \\
\hline NERVOUS SYSTEM (NS) \& SENSE ORGANS & $320-389$ & 0 & 36 & 923 & 3,165 \\
\hline CIRCULATORY SYSTEM & $390-459$ & 72 & 60 & 1,771 & 5,091 \\
\hline RESPIRATORY SYSTEM & 460-519 & 24 & 95 & 4,881 & 9,697 \\
\hline DIGESTIVE SYSTEM & 520-579 & 4 & 48 & 1,675 & 5,062 \\
\hline GENITOURINARY SYSTEM & $580-629$ & 0 & 61 & 815 & 2,284 \\
\hline SKIN \& SUBCUTANEOUS TISSUE & 680-709 & 0 & 57 & 313 & 598 \\
\hline $\begin{array}{l}\text { MUSCULOSKELETAL \& CONNECTIVE } \\
\text { TISSUE }\end{array}$ & 710-739 & 0 & 741 & 4,137 & 11,325 \\
\hline CONGENITAL ANOMALIES & $740-759$ & 0 & 0 & 0 & 4 \\
\hline $\begin{array}{l}\text { SYMPTOMS, SIGNS, \& ILL-DEFINED } \\
\text { CONDITIONS }\end{array}$ & 780-799 & 15 & 77 & 2,181 & 4,991 \\
\hline INJURY \& POISONING & 800-999 & 102 & 190 & 2,240 & 6,750 \\
\hline
\end{tabular}

\footnotetext{
*Absences with >1 ICD-9-CM code in the same diagnostic category were counted only once. Only those diagnostic categories and gender/job category combinations with at least one occurrence appear in this table.
} 


\section{Savannah River Site 2008}

\section{Absence Data}

Appendix K. Age-Adjusted IIIness and Injury Rates by Diagnostic Category*

Part 1. Men

\begin{tabular}{|c|c|c|c|c|c|}
\hline & & $\begin{array}{l}\text { Number of } \\
\text { Diagnoses }\end{array}$ & $\begin{array}{c}\text { Age-Adjusted } \\
\text { Rate per } 1,000 * *\end{array}$ & $\begin{array}{c}\text { Lower } 95 \% \\
\text { Confidence } \\
\text { Limit per 1,000 }\end{array}$ & $\begin{array}{c}\text { Upper } 95 \% \\
\text { Confidence } \\
\text { Limit per 1,000 }\end{array}$ \\
\hline Diagnostic Category & ICD-9-CM Code & \multirow[b]{2}{*}{145} & \multirow[b]{2}{*}{17.3} & \multirow[b]{2}{*}{13.1} & \multirow[b]{2}{*}{22.7} \\
\hline INFECTIOUS \& PARASITIC DISEASES (DIS) & 001-139 & & & & \\
\hline -Intestinal Infectious Dis & 001-009 & 72 & 9.3 & 6.3 & 13.7 \\
\hline -Other Bacterial Dis & 030-041 & 49 & 5.8 & 3.6 & 9.4 \\
\hline -Viral Dis with Exanthem & $050-057$ & 7 & 0.4 & 0.2 & 1.0 \\
\hline -Arthropod-Borne Viral Dis & $060-066$ & 1 & 0.6 & 0.1 & 4.0 \\
\hline -Other Viral Dis \& Chlamydiae & 070-079 & 8 & 0.6 & 0.3 & 1.3 \\
\hline -Rickettsioses \& Other Arthropod & 080-088 & 3 & 0.3 & 0.1 & 0.8 \\
\hline -Mycoses & $110-118$ & 4 & 0.2 & 0.1 & 0.6 \\
\hline -Other Infections \& Parasitic Dis & $130-136$ & 1 & 0.1 & 0.0 & 0.4 \\
\hline MALIGNANT NEOPLASMS & $140-208,230-234$ & 97 & 7.4 & 6.0 & 9.2 \\
\hline -Lip, Oral Cavity, Pharynx & 140-149 & 6 & 0.4 & 0.2 & 1.0 \\
\hline -Digestive \& Peritoneal & 150-159 & 14 & 0.9 & 0.5 & 1.5 \\
\hline -Respiratory \& Intrathoracic & $160-165$ & 5 & 0.6 & 0.2 & 1.4 \\
\hline -Bone, Connective Tissue, Skin & $170-173,176$ & 15 & 1.4 & 0.8 & 2.3 \\
\hline -Genitourinary & 179-189 & 26 & 1.8 & 1.2 & 2.7 \\
\hline -Other \& Unspecified Sites & 190, 193-199 & 20 & 1.7 & 1.0 & 2.7 \\
\hline -Nervous System & 191-192 & 3 & 0.3 & 0.1 & 0.8 \\
\hline -Lymphatic \& Hematopoietic & $200-208$ & 5 & 0.3 & 0.1 & 0.8 \\
\hline -Carcinoma in situ & $230-234$ & 3 & 0.3 & 0.1 & 0.8 \\
\hline BENIGN \& UNCERTAIN NEOPLASMS & $210-229,235-239$ & 30 & 5.6 & 3.3 & 9.6 \\
\hline ENDOCRINE/METABOLIC/IMMUNITY & $240-279$ & 128 & 10.4 & 8.4 & 13.0 \\
\hline -Thyroid Gland Disorders & $240-246$ & 9 & 1.1 & 0.4 & 3.2 \\
\hline -Other Endocrine Gland Dis & $250-259$ & 48 & 4.1 & 2.8 & 6.0 \\
\hline -Other Metabolic \& Immunity Disorders & 270-279 & 71 & 5.2 & 4.1 & 6.7 \\
\hline BLOOD \& BLOOD-FORMING ORGANS & $280-289$ & 8 & 0.5 & 0.2 & 1.0 \\
\hline MENTAL DISORDERS & 290-319 & 49 & 6.9 & 4.4 & 10.8 \\
\hline -Psychoses & 290-299 & 2 & 0.1 & 0.0 & 0.6 \\
\hline -Non-Psychotic Disorders & $300-302,306-316$ & 43 & 6.5 & 4.0 & 10.4 \\
\hline -Alcohol Dependence & 303 & 3 & 0.2 & 0.1 & 0.7 \\
\hline -Drug Dependence & 304-305 & 1 & 0.1 & 0.0 & 0.4 \\
\hline NERVOUS SYSTEM (NS) \& SENSE ORGANS & 320-389 & 206 & 21.5 & 17.2 & 26.8 \\
\hline -Hereditary/Degenerative Central NS Dis & 330-337 & 8 & 1.0 & 0.3 & 3.2 \\
\hline -Other Disorders of Central NS & 340-349 & 22 & 2.2 & 1.2 & 4.0 \\
\hline -Disorders of Peripheral NS & $350-359$ & 31 & 2.4 & 1.7 & 3.5 \\
\hline -Disorders of Eye & $360-379$ & 87 & 10.9 & 7.6 & 15.6 \\
\hline
\end{tabular}

(Continued)

*Only those diagnostic categories with at least one occurrence appear in this table.

**Standardized to age distribution of 2000 U.S. population. 


\section{Savannah River Site 2008}

\section{Absence Data}

Appendix K. Age-Adjusted IIIness and Injury Rates by Diagnostic Category*

Part 1. Men

\begin{tabular}{|c|c|c|c|c|c|}
\hline & & $\begin{array}{c}\text { Number of } \\
\text { Diagnoses }\end{array}$ & $\begin{array}{c}\text { Age-Adjusted } \\
\text { Rate per } 1,000^{* * *}\end{array}$ & $\begin{array}{c}\text { Lower } 95 \% \\
\text { Confidence } \\
\text { Limit per 1,000 }\end{array}$ & $\begin{array}{c}\text { Upper } 95 \% \\
\text { Confidence } \\
\text { Limit per 1,000 }\end{array}$ \\
\hline Diagnostic Category & ICD-9-CM Code & \multirow[b]{2}{*}{54} & \multirow[b]{2}{*}{4.8} & \multirow[b]{2}{*}{3.2} & \multirow[b]{2}{*}{7.1} \\
\hline -Dis of Ear \& Mastoid & 380-389 & & & & \\
\hline CIRCULATORY SYSTEM & $390-459$ & 277 & 23.6 & 20.3 & 27.4 \\
\hline -Hypertensive Dis & $401-405$ & 97 & 8.3 & 6.8 & 10.3 \\
\hline -Ischemic Heart Dis & $410-414$ & 64 & 4.7 & 3.6 & 6.0 \\
\hline -Dis of Pulmonary Circulation & $415-417$ & 1 & 0.1 & 0.0 & 0.9 \\
\hline -Other Heart Dis & $420-429$ & 36 & 2.7 & 1.9 & 3.7 \\
\hline -Cerebrovascular Dis & $430-438$ & 18 & 2.7 & 1.3 & 5.9 \\
\hline -Dis of Arteries \& Capillaries & $440-448$ & 7 & 0.5 & 0.2 & 1.1 \\
\hline -Dis of Veins, Lymphatics, Other & 451-459 & 54 & 4.6 & 3.3 & 6.5 \\
\hline RESPIRATORY SYSTEM & $460-519$ & 906 & 92.0 & 83.0 & 101.9 \\
\hline -Acute Respiratory Infections & $460-466$ & 368 & 36.8 & 31.3 & 43.2 \\
\hline -Other Dis Upper Respiratory Tract & $470-478$ & 257 & 30.4 & 24.9 & 36.9 \\
\hline -Pneumonia \& Influenza & $480-487$ & 251 & 22.7 & 18.9 & 27.3 \\
\hline -Chronic Obstructive Dis & $490-496$ & 14 & 1.0 & 0.6 & $\overline{1.6}$ \\
\hline -Lung Dis from External Agents & $500-508$ & 7 & 0.5 & 0.2 & 1.1 \\
\hline -Other Respiratory Dis & $510-519$ & 9 & 0.7 & 0.3 & 1.4 \\
\hline DIGESTIVE SYSTEM & $520-579$ & 332 & 27.8 & 24.2 & 31.9 \\
\hline -Oral Cavity, Saliva Glands, Jaw & $520-529$ & 37 & 3.7 & 2.5 & 5.7 \\
\hline -Esophagus, Stomach, Duodenum & $530-537$ & 73 & 5.8 & 4.1 & 8.3 \\
\hline -Appendicitis & $540-543$ & 8 & 0.5 & 0.2 & 1.0 \\
\hline -Hernia & $550-553$ & 60 & 4.8 & 3.6 & 6.2 \\
\hline -Enteritis, Colitis & $555-558$ & 26 & 2.0 & 1.3 & 3.0 \\
\hline -Other Intestinal Dis & $560-569$ & 87 & 8.0 & 6.1 & 10.6 \\
\hline -Other Digestive Dis & $570-579$ & 41 & 3.0 & 2.2 & 4.1 \\
\hline GENITOURINARY SYSTEM & $580-629$ & 187 & 15.4 & 12.9 & 18.4 \\
\hline -Nephritis, Nephrosis & $580-589$ & 8 & 0.6 & 0.3 & 1.3 \\
\hline -Other Urinary Dis & $590-599$ & 135 & 10.4 & 8.3 & 13.1 \\
\hline -Male Genital Organ Dis & $600-608$ & 42 & 4.2 & 3.1 & 5.8 \\
\hline -Breast Disorders & $610-611$ & 2 & 0.1 & 0.0 & 0.6 \\
\hline SKIN \& SUBCUTANEOUS TISSUE & $680-709$ & 43 & 4.4 & 2.7 & 7.3 \\
\hline -Infections & $680-686$ & 10 & 1.5 & 0.5 & 4.7 \\
\hline -Other Inflammatory Conditions & $690-698$ & 11 & 0.8 & 0.4 & 1.5 \\
\hline -Other & 700-709 & 22 & 2.2 & 1.2 & 4.0 \\
\hline $\begin{array}{l}\text { MUSCULOSKELETAL \& CONNECTIVE } \\
\text { TISSUE }\end{array}$ & $710-739$ & 643 & 62.3 & 55.6 & 69.9 \\
\hline -Arthropathies & $710-719$ & 159 & 17.0 & 13.2 & 21.9 \\
\hline
\end{tabular}

(Continued)

* Only those diagnostic categories with at least one occurrence appear in this table.

**Standardized to age distribution of 2000 U.S. population. 


\section{Savannah River Site 2008}

\section{Absence Data}

Appendix K. Age-Adjusted IIIness and Injury Rates by Diagnostic Category*

Part 1. Men

\begin{tabular}{|c|c|c|c|c|c|}
\hline & & $\begin{array}{l}\text { Number of } \\
\text { Diagnoses }\end{array}$ & $\begin{array}{c}\text { Age-Adjusted } \\
\text { Rate per } 1,000 * *\end{array}$ & $\begin{array}{c}\text { Lower } 95 \% \\
\text { Confidence } \\
\text { Limit per 1,000 }\end{array}$ & $\begin{array}{c}\text { Upper } 95 \% \\
\text { Confidence } \\
\text { Limit per } 1,000\end{array}$ \\
\hline Diagnostic Category & ICD-9-CM Code & \multirow[b]{2}{*}{390} & \multirow[b]{2}{*}{34.5} & \multirow[b]{2}{*}{30.3} & \multirow[b]{2}{*}{39.2} \\
\hline -Dorsopathies & $720-724$ & & & & \\
\hline -Rheumatism, Excluding Back & 725-729 & 76 & 8.6 & 5.9 & 12.4 \\
\hline -Other Dis \& Acquired Deformities & 730-739 & 18 & 2.3 & 1.1 & 4.8 \\
\hline CONGENITAL ANOMALIES & $740-759$ & 1 & 0.1 & 0.0 & 0.4 \\
\hline $\begin{array}{l}\text { SYMPTOMS, SIGNS, \& ILL-DEFINED } \\
\text { CONDITIONS }\end{array}$ & 780-799 & 440 & 51.6 & 44.7 & 59.6 \\
\hline -Symptoms & 780-789 & 431 & 50.8 & 43.9 & 58.7 \\
\hline -Non-Specific Abnormal Findings & $790-796$ & 9 & 0.8 & 0.4 & 1.6 \\
\hline INJURY \& POISONING & $800-999$ & 352 & 38.0 & 32.2 & 44.8 \\
\hline -Fracture - Skull & 800-804 & 12 & 1.3 & 0.5 & 3.2 \\
\hline -Fracture - Upper Limb & 810-819 & 20 & 1.3 & 0.8 & 2.1 \\
\hline -Fracture - Lower Limb & 820-829 & 15 & 2.9 & 1.3 & 6.5 \\
\hline -Dislocation & 830-839 & 45 & 6.8 & 4.3 & 10.7 \\
\hline -Sprains \& Strains - Back & 846-847 & 40 & 3.8 & 2.5 & 5.7 \\
\hline -Sprains \& Strains - Other & $840-845,848$ & 65 & 7.9 & 5.4 & 11.6 \\
\hline -Intracranial Injury & $850-854$ & 5 & 0.5 & 0.2 & 1.2 \\
\hline -Internal Injury - Thorax, Abdomen, Pelvis & $860-869$ & 7 & 0.5 & 0.2 & 1.0 \\
\hline -Open Wound - Head, Neck, Trunk & $870-879$ & 16 & 1.2 & 0.7 & 2.0 \\
\hline -Late Effects of Accident & 905-909 & 1 & 0.1 & 0.0 & 0.6 \\
\hline -Superficial Injury & 910-919 & 10 & 1.8 & 0.7 & 4.4 \\
\hline -Contusion & \begin{tabular}{|l|}
$920-924$ \\
\end{tabular} & 32 & 2.9 & 1.8 & 4.7 \\
\hline -Foreign Body Entering Orifice & 930-939 & 2 & 0.1 & 0.0 & 0.6 \\
\hline -Burns & 940-949 & 6 & 0.3 & 0.2 & 0.7 \\
\hline -Injury to Nerves \& Spinal Cord & $950-957$ & 2 & 0.2 & 0.0 & 0.7 \\
\hline -Complications \& Unspecified Injuries & 958-959 & 1 & 0.1 & 0.0 & 0.4 \\
\hline -Poisoning - Medicinal/Biological & $960-979$ & 2 & 0.1 & 0.0 & 0.4 \\
\hline -Unspecified Effects - External Causes & $990-995$ & 10 & 0.8 & 0.4 & 1.5 \\
\hline -Complications of Surgical/Medical Care & 996-999 & 61 & 5.5 & 3.6 & 8.3 \\
\hline Total & & 3844 & 384.8 & 366.6 & 403.8 \\
\hline
\end{tabular}

* Only those diagnostic categories with at least one occurrence appear in this table.

**Standardized to age distribution of 2000 U.S. population. 


\section{Savannah River Site 2008}

\section{Absence Data}

Appendix K. Age-Adjusted IIIness and Injury Rates by Diagnostic Category*

Part 2. Women

\begin{tabular}{|c|c|c|c|c|c|}
\hline & & $\begin{array}{l}\text { Number of } \\
\text { Diagnoses }\end{array}$ & $\begin{array}{c}\text { Age-Adjusted } \\
\text { Rate per } 1,000 * *\end{array}$ & $\begin{array}{c}\text { Lower } 95 \% \\
\text { Confidence } \\
\text { Limit per 1,000 }\end{array}$ & $\begin{array}{c}\text { Upper } 95 \% \\
\text { Confidence } \\
\text { Limit per 1,000 }\end{array}$ \\
\hline Diagnostic Category & ICD-9-CM Code & & & & \\
\hline INFECTIOUS \& PARASITIC DISEASES (DIS) & 001-139 & 70 & 31.3 & 21.9 & 44.6 \\
\hline -Intestinal Infectious Dis & 001-009 & 25 & 12.9 & 7.3 & 22.9 \\
\hline -Other Bacterial Dis & $030-041$ & 15 & 4.9 & 2.2 & 10.6 \\
\hline -Viral Dis with Exanthem & 050-057 & 7 & 4.3 & 1.5 & 12.3 \\
\hline -Other Viral Dis \& Chlamydiae & 070-079 & 13 & 6.9 & 3.1 & 15.5 \\
\hline -Mycoses & $110-118$ & 7 & 1.6 & 0.8 & 3.4 \\
\hline -Other Infections \& Parasitic Dis & $130-136$ & 3 & 0.6 & 0.2 & 1.8 \\
\hline MALIGNANT NEOPLASMS & $140-208,230-234$ & 31 & 7.4 & 5.0 & 10.9 \\
\hline -Digestive \& Peritoneal & 150-159 & 5 & 1.2 & 0.5 & 2.8 \\
\hline -Bone, Connective Tissue, Skin & 170-173, 176 & 1 & 0.2 & 0.0 & 1.7 \\
\hline -Breast & 174-175 & 12 & 2.4 & 1.4 & $\overline{4.2}$ \\
\hline -Genitourinary & 179-189 & 1 & 0.2 & 0.0 & 1.7 \\
\hline -Other \& Unspecified Sites & 190, 193-199 & 11 & 2.6 & 1.4 & 4.7 \\
\hline -Lymphatic \& Hematopoietic & $200-208$ & 1 & 0.8 & 0.1 & 5.7 \\
\hline BENIGN \& UNCERTAIN NEOPLASMS & 210-229, 235-239 & 34 & 10.9 & 6.7 & 17.7 \\
\hline ENDOCRINE/METABOLIC/IMMUNITY & $240-279$ & 50 & 18.6 & 12.5 & 27.9 \\
\hline -Thyroid Gland Disorders & $240-246$ & 11 & 3.6 & 1.4 & 9.2 \\
\hline -Other Endocrine Gland Dis & $250-259$ & 21 & 11.0 & 6.1 & 19.8 \\
\hline -Nutritional Deficiencies & $260-269$ & 2 & 0.5 & 0.1 & 1.9 \\
\hline -Other Metabolic \& Immunity Disorders & 270-279 & 16 & 3.5 & 2.2 & 5.7 \\
\hline BLOOD \& BLOOD-FORMING ORGANS & 280-289 & 29 & 6.6 & 4.6 & 9.6 \\
\hline MENTAL DISORDERS & 290-319 & 32 & 7.6 & 5.2 & 11.1 \\
\hline -Psychoses & 290-299 & 3 & 0.6 & 0.2 & 1.9 \\
\hline -Non-Psychotic Disorders & $300-302,306-316$ & 25 & 6.0 & 3.9 & 9.3 \\
\hline -Alcohol Dependence & 303 & 2 & 0.5 & 0.1 & 1.9 \\
\hline -Drug Dependence & 304-305 & 2 & 0.5 & 0.1 & 1.9 \\
\hline NERVOUS SYSTEM (NS) \& SENSE ORGANS & 320-389 & 138 & 53.4 & 41.5 & 68.9 \\
\hline -Hereditary/Degenerative Central NS Dis & 330-337 & 1 & 0.2 & 0.0 & 1.7 \\
\hline -Other Disorders of Central NS & 340-349 & 34 & 10.4 & 6.4 & 16.9 \\
\hline -Disorders of Peripheral NS & $350-359$ & 24 & 5.7 & 3.7 & 8.9 \\
\hline -Disorders of Eye & $360-379$ & 51 & 24.1 & 16.1 & 36.0 \\
\hline -Dis of Ear \& Mastoid & $380-389$ & 28 & 13.0 & 7.3 & 23.2 \\
\hline CIRCULATORY SYSTEM & \begin{tabular}{|l|}
$390-459$ \\
\end{tabular} & 108 & 39.3 & 29.6 & 52.3 \\
\hline -Hypertensive Dis & 401-405 & 57 & 19.6 & 13.4 & 28.7 \\
\hline -Ischemic Heart Dis & $410-414$ & 2 & 0.4 & 0.1 & 1.6 \\
\hline
\end{tabular}

(Continued)

*Only those diagnostic categories with at least one occurrence appear in this table.

**Standardized to age distribution of 2000 U.S. population. 


\section{Savannah River Site 2008}

\section{Absence Data}

Appendix K. Age-Adjusted IIIness and Injury Rates by Diagnostic Category*

Part 2. Women

\begin{tabular}{|c|c|c|c|c|c|}
\hline & & $\begin{array}{c}\text { Number of } \\
\text { Diagnoses }\end{array}$ & $\begin{array}{c}\text { Age-Adjusted } \\
\text { Rate per } 1,000 * *\end{array}$ & \begin{tabular}{c|} 
Lower $95 \%$ \\
Confidence \\
Limit per 1,000
\end{tabular} & $\begin{array}{c}\text { Upper } 95 \% \\
\text { Confidence } \\
\text { Limit per 1,000 }\end{array}$ \\
\hline Diagnostic Category & ICD-9-CM Code & \multirow[b]{2}{*}{2} & \multirow[b]{2}{*}{1.0} & \multirow[b]{2}{*}{0.2} & \multirow[b]{2}{*}{5.0} \\
\hline -Dis of Pulmonary Circulation & $415-417$ & & & & \\
\hline -Other Heart Dis & $420-429$ & 18 & 4.1 & 2.4 & 7.1 \\
\hline -Cerebrovascular Dis & $430-438$ & 2 & 0.4 & 0.1 & 1.6 \\
\hline -Dis of Veins, Lymphatics, Other & 451-459 & 27 & 13.8 & 7.8 & 24.4 \\
\hline RESPIRATORY SYSTEM & $460-519$ & 530 & 199.4 & 175.3 & 226.9 \\
\hline -Acute Respiratory Infections & $460-466$ & 247 & 95.9 & 79.5 & 115.6 \\
\hline -Other Dis Upper Respiratory Tract & $470-478$ & 140 & 56.7 & 44.0 & 73.1 \\
\hline -Pneumonia \& Influenza & $480-487$ & 108 & 34.0 & 25.7 & 44.9 \\
\hline -Chronic Obstructive Dis & $490-496$ & 19 & 9.5 & 4.9 & 18.8 \\
\hline -Lung Dis from External Agents & $500-508$ & 3 & 0.6 & 0.2 & 1.9 \\
\hline -Other Respiratory Dis & $510-519$ & 13 & 2.7 & 1.5 & 4.6 \\
\hline DIGESTIVE SYSTEM & $520-579$ & 153 & 66.6 & 53.0 & 83.8 \\
\hline -Oral Cavity, Saliva Glands, Jaw & $520-529$ & 38 & 9.0 & 6.4 & 12.7 \\
\hline -Esophagus, Stomach, Duodenum & $530-537$ & 23 & 10.2 & 5.6 & 18.6 \\
\hline -Appendicitis & $540-543$ & 1 & 0.2 & 0.0 & 1.7 \\
\hline -Hernia & $550-553$ & 13 & 7.5 & 3.5 & 16.2 \\
\hline -Enteritis, Colitis & $555-558$ & 14 & 6.2 & 2.8 & 14.1 \\
\hline -Other Intestinal Dis & $560-569$ & 19 & 11.5 & 6.1 & 21.7 \\
\hline -Other Digestive Dis & $570-579$ & 45 & 22.0 & 14.9 & 32.5 \\
\hline GENITOURINARY SYSTEM & $580-629$ & 199 & 82.5 & 66.7 & 102.0 \\
\hline -Nephritis, Nephrosis & $580-589$ & 8 & 1.6 & 0.8 & 3.3 \\
\hline -Other Urinary Dis & $590-599$ & 61 & 21.8 & 15.0 & 31.6 \\
\hline -Breast Disorders & 610-611 & 6 & 1.3 & 0.6 & 3.0 \\
\hline -Pelvic Inflammatory Dis & 614-616 & 16 & 10.5 & 5.3 & 20.7 \\
\hline -Other Female Disorders & $617-629$ & 108 & 47.3 & 35.4 & 63.2 \\
\hline PREGNANCY \& CHILDBIRTH & $630-677$ & 4 & 6.4 & 2.4 & 17.0 \\
\hline -Pregnancy with Abortive Outcome & 634-639 & 4 & 6.4 & 2.4 & 17.0 \\
\hline SKIN \& SUBCUTANEOUS TISSUE & $680-709$ & 23 & 11.2 & 6.2 & 20.4 \\
\hline -Infections & $680-686$ & 10 & 3.6 & 1.4 & 9.2 \\
\hline -Other & 700-709 & 13 & 7.6 & 3.6 & 16.3 \\
\hline $\begin{array}{l}\text { MUSCULOSKELETAL \& CONNECTIVE } \\
\text { TISSUE }\end{array}$ & $710-739$ & 439 & 165.0 & 143.4 & 189.9 \\
\hline -Arthropathies & 710-719 & 78 & 24.7 & 17.9 & 34.1 \\
\hline -Dorsopathies & $720-724$ & 272 & 113.0 & 94.5 & 135.3 \\
\hline -Rheumatism, Excluding Back & 725-729 & 73 & 23.2 & 16.8 & 32.1 \\
\hline -Other Dis \& Acquired Deformities & $730-739$ & 16 & 4.0 & 2.3 & 7.1 \\
\hline
\end{tabular}

(Continued)

* Only those diagnostic categories with at least one occurrence appear in this table.

**Standardized to age distribution of 2000 U.S. population. 


\section{Savannah River Site 2008}

\section{Absence Data}

Appendix K. Age-Adjusted IIIness and Injury Rates by Diagnostic Category*

Part 2. Women

\begin{tabular}{|c|c|c|c|c|c|}
\hline & & $\begin{array}{l}\text { Number of } \\
\text { Diagnoses }\end{array}$ & $\begin{array}{c}\text { Age-Adjusted } \\
\text { Rate per } 1,000 * *\end{array}$ & \begin{tabular}{|c|} 
Lower $95 \%$ \\
Confidence \\
Limit per 1,000
\end{tabular} & $\begin{array}{c}\text { Upper } 95 \% \\
\text { Confidence } \\
\text { Limit per 1,000 }\end{array}$ \\
\hline Diagnostic Category & ICD-9-CM Code & & & & \\
\hline $\begin{array}{l}\text { SYMPTOMS, SIGNS, \& ILL-DEFINED } \\
\text { CONDITIONS }\end{array}$ & 780-799 & 242 & 72.7 & 60.7 & 87.0 \\
\hline -Symptoms & 780-789 & 232 & 70.0 & 58.2 & 84.2 \\
\hline -Non-Specific Abnormal Findings & $790-796$ & 8 & 2.3 & 1.0 & 5.3 \\
\hline -Ill-Defined \& Unknown Causes & 797-799 & 2 & 0.4 & 0.1 & 1.6 \\
\hline INJURY \& POISONING & $800-999$ & 149 & 52.8 & 41.6 & 67.0 \\
\hline -Fracture - Skull & $800-804$ & 7 & 2.0 & 0.8 & 5.0 \\
\hline -Fracture - Upper Limb & 810-819 & 13 & 5.7 & 2.4 & 13.8 \\
\hline -Fracture - Lower Limb & $820-829$ & 17 & 5.0 & 2.5 & 10.2 \\
\hline -Dislocation & $830-839$ & 10 & 2.1 & 1.1 & 3.9 \\
\hline -Sprains \& Strains - Back & 846-847 & 19 & 5.5 & 2.8 & 10.6 \\
\hline -Sprains \& Strains - Other & $840-845,848$ & 16 & 3.4 & 2.1 & 5.6 \\
\hline -Intracranial Injury & $850-854$ & 2 & 0.4 & 0.1 & 1.6 \\
\hline -Open Wound - Head, Neck, Trunk & $870-879$ & 10 & 3.6 & 1.4 & 9.2 \\
\hline -Superficial Injury & 910-919 & 4 & 3.6 & 1.1 & 12.4 \\
\hline -Contusion & $920-924$ & 18 & 5.2 & 2.6 & 10.3 \\
\hline -Unspecified Effects - External Causes & 990-995 & 9 & 3.6 & 1.3 & 9.9 \\
\hline -Complications of Surgical/Medical Care & 996-999 & 24 & 12.6 & 7.6 & 21.1 \\
\hline Total & & 2231 & 831.7 & 781.4 & 885.3 \\
\hline
\end{tabular}

*Only those diagnostic categories with at least one occurrence appear in this table.

**Standardized to age distribution of 2000 U.S. population. 


\section{Savannah River Site 2008}

\section{Absence Data}

Appendix K. Age-Adjusted IIIness and Injury Rates by Diagnostic Category*

Part 3. Men and Women

\begin{tabular}{|c|c|c|c|c|c|}
\hline & & $\begin{array}{l}\text { Number of } \\
\text { Diagnoses }\end{array}$ & $\begin{array}{c}\text { Age-Adjusted } \\
\text { Rate per 1,000** }\end{array}$ & \begin{tabular}{|c|} 
Lower $95 \%$ \\
Confidence \\
Limit per 1,000
\end{tabular} & $\begin{array}{c}\text { Upper } 95 \% \\
\text { Confidence } \\
\text { Limit per 1,000 }\end{array}$ \\
\hline Diagnostic Category & ICD-9-CM Code & \multirow[b]{2}{*}{215} & \multirow[b]{2}{*}{20.8} & \multirow[b]{2}{*}{16.8} & \multirow[b]{2}{*}{25.8} \\
\hline INFECTIOUS \& PARASITIC DISEASES (DIS) & 001-139 & & & & \\
\hline -Intestinal Infectious Dis & 001-009 & 97 & 10.1 & 7.4 & 13.9 \\
\hline -Other Bacterial Dis & 030-041 & 64 & 5.6 & 3.7 & 8.4 \\
\hline -Viral Dis with Exanthem & 050-057 & 14 & 1.5 & 0.6 & 3.3 \\
\hline -Arthropod-Borne Viral Dis & $060-066$ & 1 & 0.4 & 0.1 & 2.9 \\
\hline -Other Viral Dis \& Chlamydiae & 070-079 & 21 & 2.3 & 1.2 & 4.4 \\
\hline -Rickettsioses \& Other Arthropod & 080-088 & 3 & 0.2 & 0.1 & 0.6 \\
\hline -Mycoses & 110-118 & 11 & 0.6 & 0.3 & 1.1 \\
\hline -Other Infections \& Parasitic Dis & $130-136$ & 4 & 0.2 & 0.1 & 0.5 \\
\hline MALIGNANT NEOPLASMS & $140-208,230-234$ & 128 & 7.9 & 6.5 & 9.5 \\
\hline -Lip, Oral Cavity, Pharynx & 140-149 & 6 & 0.3 & 0.1 & 0.8 \\
\hline -Digestive \& Peritoneal & 150-159 & 19 & 1.0 & 0.6 & 1.6 \\
\hline -Respiratory \& Intrathoracic & $160-165$ & 5 & 0.5 & 0.2 & 1.3 \\
\hline -Bone, Connective Tissue, Skin & $170-173,176$ & 16 & 1.2 & 0.7 & 2.0 \\
\hline -Breast & $174-175$ & 12 & 0.5 & 0.3 & 1.0 \\
\hline -Genitourinary & 179-189 & 27 & 1.5 & 1.0 & 2.2 \\
\hline -Other \& Unspecified Sites & 190, 193-199 & 31 & 2.1 & 1.4 & 3.0 \\
\hline -Nervous System & 191-192 & 3 & 0.2 & 0.1 & 0.6 \\
\hline -Lymphatic \& Hematopoietic & 200-208 & 6 & 0.4 & 0.1 & 0.8 \\
\hline -Carcinoma in situ & 230-234 & 3 & 0.2 & 0.1 & 0.6 \\
\hline BENIGN \& UNCERTAIN NEOPLASMS & 210-229, 235-239 & 64 & 7.0 & 4.8 & 10.1 \\
\hline ENDOCRINE/METABOLIC/IMMUNITY & 240-279 & 178 & 12.5 & 10.2 & 15.3 \\
\hline -Thyroid Gland Disorders & $240-246$ & 20 & 1.7 & 0.9 & 3.5 \\
\hline -Other Endocrine Gland Dis & 250-259 & 69 & 5.7 & 4.1 & 8.0 \\
\hline -Nutritional Deficiencies & $260-269$ & 2 & 0.1 & 0.0 & 0.5 \\
\hline -Other Metabolic \& Immunity Disorders & 270-279 & 87 & 4.9 & 4.0 & 6.1 \\
\hline BLOOD \& BLOOD-FORMING ORGANS & 280-289 & 37 & 2.1 & 1.5 & 2.9 \\
\hline MENTAL DISORDERS & 290-319 & 81 & 7.0 & 5.0 & 9.8 \\
\hline -Psychoses & 290-299 & 5 & 0.3 & 0.1 & 0.6 \\
\hline -Non-Psychotic Disorders & $300-302,306-316$ & 68 & 6.3 & 4.4 & 9.1 \\
\hline -Alcohol Dependence & 303 & 5 & 0.3 & 0.1 & 0.7 \\
\hline -Drug Dependence & 304-305 & 3 & 0.2 & 0.1 & 0.5 \\
\hline NERVOUS SYSTEM (NS) \& SENSE ORGANS & 320-389 & 344 & 29.7 & 25.2 & 35.1 \\
\hline -Hereditary/Degenerative Central NS Dis & 330-337 & 9 & 0.9 & 0.3 & 2.4 \\
\hline -Other Disorders of Central NS & $340-349$ & 56 & 4.3 & 2.9 & 6.3 \\
\hline
\end{tabular}

(Continued)

* Only those diagnostic categories with at least one occurrence appear in this table.

**Standardized to age distribution of 2000 U.S. population. 


\section{Savannah River Site 2008}

\section{Absence Data}

Appendix K. Age-Adjusted IIIness and Injury Rates by Diagnostic Category*

Part 3. Men and Women

\begin{tabular}{|c|c|c|c|c|c|}
\hline & & $\begin{array}{l}\text { Number of } \\
\text { Diagnoses }\end{array}$ & $\begin{array}{c}\text { Age-Adjusted } \\
\text { Rate per } 1,000 * *\end{array}$ & $\begin{array}{c}\text { Lower } 95 \% \\
\text { Confidence } \\
\text { Limit per 1,000 }\end{array}$ & $\begin{array}{c}\text { Upper } 95 \% \\
\text { Confidence } \\
\text { Limit per 1,000 }\end{array}$ \\
\hline Diagnostic Category & ICD-9-CM Code & \multirow[b]{2}{*}{55} & \multirow[b]{2}{*}{3.2} & \multirow[b]{2}{*}{2.4} & \multirow[b]{2}{*}{4.3} \\
\hline -Disorders of Peripheral NS & $350-359$ & & & & \\
\hline -Disorders of Eye & $360-379$ & 138 & 14.0 & 10.8 & 18.2 \\
\hline -Dis of Ear \& Mastoid & $380-389$ & 82 & 7.2 & 5.0 & 10.3 \\
\hline CIRCULATORY SYSTEM & $390-459$ & 385 & 28.0 & 24.4 & 32.2 \\
\hline -Hypertensive Dis & $401-405$ & 154 & 11.5 & 9.3 & 14.1 \\
\hline -Ischemic Heart Dis & $410-414$ & 66 & 3.7 & 2.9 & 4.8 \\
\hline -Dis of Pulmonary Circulation & $415-417$ & 3 & 0.3 & 0.1 & 0.9 \\
\hline -Other Heart Dis & $420-429$ & 54 & 2.9 & 2.2 & 3.8 \\
\hline -Cerebrovascular Dis & $430-438$ & 20 & 2.1 & 1.0 & 4.3 \\
\hline -Dis of Arteries \& Capillaries & $440-448$ & 7 & 0.4 & 0.2 & 0.8 \\
\hline -Dis of Veins, Lymphatics, Other & 451-459 & 81 & 7.2 & 5.1 & 10.0 \\
\hline RESPIRATORY SYSTEM & $460-519$ & 1436 & 118.7 & 109.5 & 128.7 \\
\hline -Acute Respiratory Infections & $460-466$ & 615 & 51.2 & 45.3 & 57.9 \\
\hline -Other Dis Upper Respiratory Tract & $470-478$ & 397 & 37.0 & 31.6 & 43.3 \\
\hline -Pneumonia \& Influenza & $480-487$ & 359 & 25.6 & 21.9 & 30.0 \\
\hline -Chronic Obstructive Dis & $490-496$ & 33 & 3.1 & 1.8 & 5.4 \\
\hline -Lung Dis from External Agents & $500-508$ & 10 & 0.5 & 0.3 & 1.1 \\
\hline -Other Respiratory Dis & $510-519$ & 22 & 1.2 & 0.8 & 1.8 \\
\hline DIGESTIVE SYSTEM & $520-579$ & 485 & 38.0 & 33.4 & 43.2 \\
\hline -Oral Cavity, Saliva Glands, Jaw & $520-529$ & 75 & 5.3 & 4.0 & 6.9 \\
\hline -Esophagus, Stomach, Duodenum & $530-537$ & 96 & 6.8 & 5.0 & 9.3 \\
\hline -Appendicitis & $540-543$ & 9 & 0.4 & 0.2 & 0.8 \\
\hline -Hernia & $550-553$ & 73 & 5.7 & 4.1 & 7.8 \\
\hline -Enteritis, Colitis & $555-558$ & 40 & 3.4 & 2.0 & 5.6 \\
\hline -Other Intestinal Dis & \begin{tabular}{|l|}
$560-569$ \\
\end{tabular} & 106 & 9.4 & 7.1 & 12.3 \\
\hline -Other Digestive Dis & $570-579$ & 86 & 7.1 & 5.2 & 9.6 \\
\hline GENITOURINARY SYSTEM & 580-629 & 386 & 34.1 & 29.2 & 39.9 \\
\hline -Nephritis, Nephrosis & $580-589$ & 16 & 0.9 & 0.5 & 1.5 \\
\hline -Other Urinary Dis & \begin{tabular}{|l|l|}
$590-599$ \\
\end{tabular} & 196 & 13.4 & 11.0 & 16.5 \\
\hline -Male Genital Organ Dis & $600-608$ & 42 & 3.6 & 2.6 & 5.0 \\
\hline -Breast Disorders & $610-611$ & 8 & 0.4 & 0.2 & 0.9 \\
\hline -Pelvic Inflammatory Dis & 614-616 & 16 & 2.7 & 1.4 & 5.4 \\
\hline -Other Female Disorders & 617-629 & 108 & 13.0 & 9.5 & 17.6 \\
\hline PREGNANCY \& CHILDBIRTH & 630-677 & 4 & 1.7 & 0.6 & 4.4 \\
\hline -Pregnancy with Abortive Outcome & 634-639 & 4 & 1.7 & 0.6 & 4.4 \\
\hline
\end{tabular}

(Continued)

*Only those diagnostic categories with at least one occurrence appear in this table.

**Standardized to age distribution of 2000 U.S. population. 


\section{Savannah River Site 2008}

\section{Absence Data}

Appendix K. Age-Adjusted IIIness and Injury Rates by Diagnostic Category*

Part 3. Men and Women

\begin{tabular}{|c|c|c|c|c|c|}
\hline & & $\begin{array}{l}\text { Number of } \\
\text { Diagnoses }\end{array}$ & $\begin{array}{c}\text { Age-Adjusted } \\
\text { Rate per } 1,000 * *\end{array}$ & \begin{tabular}{|c|} 
Lower $95 \%$ \\
Confidence \\
Limit per 1,000
\end{tabular} & $\begin{array}{c}\text { Upper } 95 \% \\
\text { Confidence } \\
\text { Limit per 1,000 }\end{array}$ \\
\hline Diagnostic Category & ICD-9-CM Code & & & & \\
\hline SKIN \& SUBCUTANEOUS TISSUE & $680-709$ & 66 & 6.1 & 4.2 & 9.0 \\
\hline -Infections & $680-686$ & 20 & 1.9 & 0.9 & 4.1 \\
\hline -Other Inflammatory Conditions & $690-698$ & 11 & 0.6 & 0.3 & 1.2 \\
\hline -Other & 700-709 & 35 & 3.5 & 2.1 & 5.8 \\
\hline $\begin{array}{l}\text { MUSCULOSKELETAL \& CONNECTIVE } \\
\text { TISSUE }\end{array}$ & $710-739$ & 1082 & 87.5 & 79.9 & 95.8 \\
\hline -Arthropathies & $710-719$ & 237 & 18.5 & 15.2 & 22.7 \\
\hline -Dorsopathies & $720-724$ & 662 & 54.4 & 48.5 & 61.0 \\
\hline -Rheumatism, Excluding Back & 725-729 & 149 & 11.9 & 9.3 & 15.3 \\
\hline -Other Dis \& Acquired Deformities & 730-739 & 34 & 2.7 & 1.6 & 4.4 \\
\hline CONGENITAL ANOMALIES & $740-759$ & 1 & 0.0 & 0.0 & 0.3 \\
\hline $\begin{array}{l}\text { SYMPTOMS, SIGNS, \& ILL-DEFINED } \\
\text { CONDITIONS }\end{array}$ & 780-799 & 682 & 57.4 & 51.2 & 64.3 \\
\hline -Symptoms & 780-789 & 663 & 56.1 & 50.0 & 63.0 \\
\hline -Non-Specific Abnormal Findings & $790-796$ & 17 & 1.2 & 0.7 & 1.9 \\
\hline -Ill-Defined \& Unknown Causes & 797-799 & 2 & 0.1 & 0.0 & 0.4 \\
\hline INJURY \& POISONING & $800-999$ & 501 & 41.8 & 36.5 & 47.8 \\
\hline -Fracture - Skull & $800-804$ & 19 & 1.4 & 0.7 & 2.7 \\
\hline -Fracture - Upper Limb & 810-819 & 33 & 2.7 & 1.4 & 5.0 \\
\hline -Fracture - Lower Limb & 820-829 & 32 & 3.4 & 1.9 & 5.9 \\
\hline -Dislocation & $830-839$ & 55 & 5.6 & 3.7 & 8.4 \\
\hline -Sprains \& Strains - Back & 846-847 & 59 & 4.3 & 3.1 & 6.1 \\
\hline -Sprains \& Strains - Other & $840-845,848$ & 81 & 6.9 & 5.0 & 9.6 \\
\hline -Intracranial Injury & $850-854$ & 7 & 0.5 & 0.2 & 1.1 \\
\hline -Internal Injury - Thorax, Abdomen, Pelvis & $860-869$ & 7 & 0.4 & 0.2 & 0.9 \\
\hline -Open Wound - Head, Neck, Trunk & 870-879 & 26 & 1.9 & 1.1 & 3.2 \\
\hline -Late Effects of Accident & 905-909 & 1 & 0.1 & 0.0 & 0.4 \\
\hline -Superficial Injury & 910-919 & 14 & 2.3 & 1.1 & 4.7 \\
\hline -Contusion & $920-924$ & 50 & 3.5 & 2.4 & 5.2 \\
\hline -Foreign Body Entering Orifice & 930-939 & 2 & 0.1 & 0.0 & 0.4 \\
\hline -Burns & $940-949$ & 6 & 0.3 & 0.1 & 0.6 \\
\hline -Injury to Nerves \& Spinal Cord & $950-957$ & 2 & 0.1 & 0.0 & 0.5 \\
\hline -Complications \& Unspecified Injuries & 958-959 & 1 & 0.0 & 0.0 & 0.3 \\
\hline -Poisoning - Medicinal/Biological & $960-979$ & 2 & 0.1 & 0.0 & 0.4 \\
\hline -Unspecified Effects - External Causes & $990-995$ & 19 & 1.6 & 0.8 & 3.4 \\
\hline
\end{tabular}

(Continued)

*Only those diagnostic categories with at least one occurrence appear in this table.

**Standardized to age distribution of 2000 U.S. population. 


\section{Savannah River Site 2008}

Absence Data

Appendix K. Age-Adjusted Illness and Injury Rates by Diagnostic Category*

Part 3. Men and Women

\begin{tabular}{|c|c|c|c|c|c|}
\hline & & $\begin{array}{l}\text { Number of } \\
\text { Diagnoses }\end{array}$ & $\begin{array}{c}\text { Age-Adjusted } \\
\text { Rate per } 1,000^{* *}\end{array}$ & $\begin{array}{c}\text { Lower } 95 \% \\
\text { Confidence } \\
\text { Limit per } 1,000\end{array}$ & $\begin{array}{c}\text { Upper } 95 \% \\
\text { Confidence } \\
\text { Limit per 1,000 }\end{array}$ \\
\hline Diagnostic Category & ICD-9-CM Code & \multirow[b]{2}{*}{85} & \multirow[b]{2}{*}{6.7} & \multirow[b]{2}{*}{4.9} & \multirow[b]{2}{*}{9.2} \\
\hline -Complications of Surgical/Medical Care & 996-999 & & & & \\
\hline Total & & 6075 & 500.4 & 481.5 & 520.0 \\
\hline
\end{tabular}

* Only those diagnostic categories with at least one occurrence appear in this table.

**Standardized to age distribution of 2000 U.S. population. 


\section{Savannah River Site 2008}

OSHA Data

Appendix L. Number of Workers with at Least One OSHA Event by Gender, Age, and Job Category*

\begin{tabular}{|c|c|c|c|c|c|c|c|c|c|c|}
\hline \multirow{3}{*}{ Job Category } & \multicolumn{4}{|c|}{ Women } & \multicolumn{5}{|c|}{ Men } & \multirow[b]{3}{*}{ TOTAL } \\
\hline & \multicolumn{3}{|c|}{ Age Group } & \multirow[b]{2}{*}{ TOTAL } & \multicolumn{4}{|c|}{ Age Group } & \multirow[b]{2}{*}{ TOTAL } & \\
\hline & $16-29$ & $40-49$ & $50+$ & & $16-29$ & 30 - 39 & 40 - 49 & $50+$ & & \\
\hline Professional & 0 & 0 & 1 & 1 & 0 & 0 & 2 & 0 & 2 & 3 \\
\hline Administrative Support & 0 & 0 & 3 & 3 & 0 & 0 & 0 & 2 & 2 & 5 \\
\hline Technical Support & 0 & 1 & 3 & 4 & 0 & 1 & 1 & 1 & 3 & 7 \\
\hline Service & 0 & 0 & 0 & 0 & 0 & 0 & 0 & 1 & 1 & 1 \\
\hline Crafts & 0 & 0 & 0 & 0 & 2 & 0 & 3 & 1 & 6 & 6 \\
\hline Line Operators & 1 & 2 & 2 & 5 & 0 & 0 & 2 & 4 & 6 & 11 \\
\hline TOTAL & 1 & 3 & 9 & 13 & 2 & 1 & 8 & 9 & 20 & 33 \\
\hline
\end{tabular}

*Only those job categories and gender/age combinations with at least one OSHA event appear in this table.

Appendix M. Total Number of Workdays Lost or with Restricted Activity from OSHA Events by Gender and Age

\begin{tabular}{|c|c|c|c|c|c|c|c|c|c|}
\hline \multirow[b]{2}{*}{ Age Group } & \multicolumn{3}{|c|}{ Women } & \multicolumn{3}{|c|}{ Men } & \multicolumn{3}{|c|}{ TOTAL } \\
\hline & $\begin{array}{c}\text { Number of } \\
\text { Events }\end{array}$ & $\begin{array}{c}\text { Days } \\
\text { Restricted }\end{array}$ & Days Lost & $\begin{array}{c}\text { Number of } \\
\text { Events }\end{array}$ & \begin{tabular}{|c|} 
Days \\
Restricted
\end{tabular} & Days Lost & \begin{tabular}{|c|} 
Number of \\
Events
\end{tabular} & $\begin{array}{c}\text { Days } \\
\text { Restricted }\end{array}$ & Days Lost \\
\hline $16-29$ & 1 & 0 & 0 & 2 & 0 & 0 & 3 & 0 & 0 \\
\hline $30-39$ & 0 & 0 & 0 & 1 & 22 & 0 & 1 & 22 & 0 \\
\hline $40-49$ & 3 & 126 & 0 & 8 & 202 & 0 & 11 & 328 & 0 \\
\hline $50+$ & 9 & 105 & 118 & 9 & 47 & 28 & 18 & 152 & 146 \\
\hline TOTAL & 13 & 231 & 118 & 20 & 271 & 28 & 33 & 502 & 146 \\
\hline
\end{tabular}

Appendix N. Total Number of Workdays Lost or with Restricted Activity from OSHA Events by Gender and Job Category*

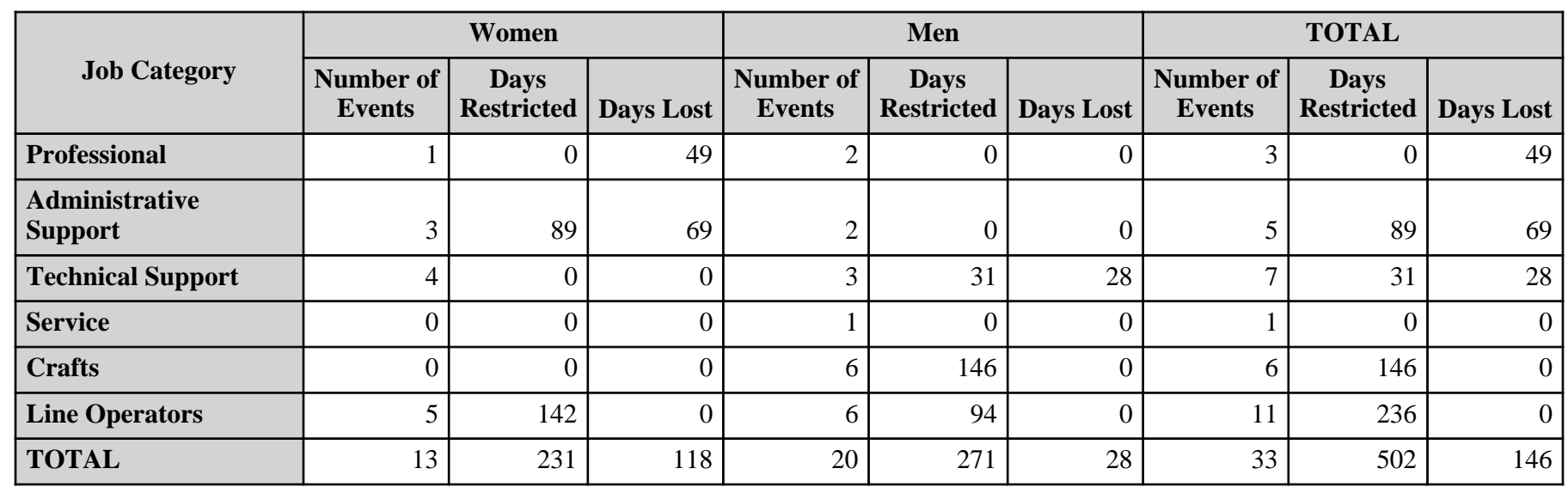

*Only those job categories with at least one OSHA event appear in this table. 


\section{Savannah River Site 2008}

OSHA Data

Appendix O. Number of Diagnoses in Each Diagnostic Category by Gender and Age*

\begin{tabular}{|c|c|c|c|c|c|}
\hline & & \multicolumn{4}{|c|}{ Women } \\
\hline & & \multicolumn{3}{|c|}{ Age Group } & \multirow[b]{2}{*}{ TOTAL } \\
\hline & & $16-29$ & $40-49$ & $50+$ & \\
\hline Diagnostic Category & ICD-9-CM code & \multirow[b]{2}{*}{0} & \multirow[b]{2}{*}{0} & \multirow[b]{2}{*}{1} & \multirow[b]{2}{*}{1} \\
\hline ENDOCRINE/METABOLIC/IMMUNITY & $240-279$ & & & & \\
\hline -Other Metabolic \& Immunity Disorders & $270-279$ & 0 & 0 & 1 & 1 \\
\hline RESPIRATORY SYSTEM & $460-519$ & 0 & 0 & 0 & 0 \\
\hline -Lung Dis from External Agents & $500-508$ & 0 & 0 & 0 & 0 \\
\hline $\begin{array}{l}\text { MUSCULOSKELETAL \& CONNECTIVE } \\
\text { TISSUE }\end{array}$ & $710-739$ & 0 & 0 & 4 & 4 \\
\hline -Arthropathies & 710-719 & 0 & 0 & 2 & 2 \\
\hline -Dorsopathies & $720-724$ & 0 & 0 & 1 & 1 \\
\hline -Rheumatism, Excluding Back & $725-729$ & 0 & 0 & 1 & 1 \\
\hline $\begin{array}{l}\text { SYMPTOMS, SIGNS, \& ILL-DEFINED } \\
\text { CONDITIONS }\end{array}$ & 780-799 & 0 & 0 & 1 & 1 \\
\hline -Symptoms & $780-789$ & 0 & 0 & 1 & 1 \\
\hline INJURY \& POISONING & 800-999 & 1 & 3 & 13 & 17 \\
\hline -Fracture - Upper Limb & 810-819 & 0 & 1 & 0 & 1 \\
\hline -Fracture - Lower Limb & $820-829$ & 0 & 0 & 2 & 2 \\
\hline -Dislocation & 830-839 & 0 & 0 & 0 & 0 \\
\hline -Sprains \& Strains - Back & 846-847 & 0 & 0 & 2 & 2 \\
\hline -Sprains \& Strains - Other & $840-845,848$ & 0 & 0 & 1 & 1 \\
\hline -Open Wound - Head, Neck, Trunk & 870-879 & 0 & 0 & 0 & 0 \\
\hline -Open Wound - Upper Limb & 880-887 & 1 & 0 & 0 & 1 \\
\hline -Open Wound - Lower Limb & 890-897 & 0 & 0 & 1 & 1 \\
\hline -Superficial Injury & 910-919 & 0 & 0 & 2 & 2 \\
\hline -Contusion & 920-924 & 0 & 0 & 4 & 4 \\
\hline -Foreign Body Entering Orifice & \begin{tabular}{|c|}
$930-939$ \\
\end{tabular} & 0 & 1 & 0 & 1 \\
\hline -Toxic Effects - Non-medicinal & $980-989$ & 0 & 1 & 0 & 1 \\
\hline -Unspecified Effects - External Causes & $990-995$ & 0 & 0 & 1 & 1 \\
\hline
\end{tabular}

*Only those diagnostic categories and gender/age combinations with at least one OSHA event appear in this table. 


\section{Savannah River Site 2008}

\section{OSHA Data}

Appendix O. Number of Diagnoses in Each Diagnostic Category by Gender and Age*

\begin{tabular}{|c|c|c|c|c|c|c|c|}
\hline & & \multicolumn{5}{|c|}{ Men } & \multirow[b]{3}{*}{ TOTAL } \\
\hline & & \multicolumn{4}{|c|}{ Age Group } & \multirow[b]{2}{*}{ TOTAL } & \\
\hline & & $16-29$ & $30-39$ & 40 - 49 & $50+$ & & \\
\hline Diagnostic Category & ICD-9-CM code & & & & & & \\
\hline ENDOCRINE/METABOLIC/IMMUNITY & $240-279$ & 0 & 0 & 0 & 0 & 0 & 1 \\
\hline -Other Metabolic \& Immunity Disorders & $270-279$ & 0 & 0 & 0 & 0 & 0 & 1 \\
\hline RESPIRATORY SYSTEM & $460-519$ & 0 & 0 & 0 & 2 & 2 & 2 \\
\hline -Lung Dis from External Agents & $500-508$ & 0 & 0 & 0 & 2 & 2 & 2 \\
\hline $\begin{array}{l}\text { MUSCULOSKELETAL \& CONNECTIVE } \\
\text { TISSUE }\end{array}$ & $710-739$ & 0 & 2 & 2 & 1 & 5 & 9 \\
\hline -Arthropathies & $710-719$ & 0 & 0 & 1 & 0 & 1 & 3 \\
\hline -Dorsopathies & $720-724$ & 0 & 1 & 0 & 0 & 1 & 2 \\
\hline -Rheumatism, Excluding Back & $725-729$ & 0 & 1 & 1 & 1 & 3 & 4 \\
\hline $\begin{array}{l}\text { SYMPTOMS, SIGNS, \& ILL-DEFINED } \\
\text { CONDITIONS }\end{array}$ & $780-799$ & 0 & 0 & 0 & 0 & 0 & 1 \\
\hline -Symptoms & $780-789$ & 0 & 0 & 0 & 0 & 0 & 1 \\
\hline INJURY \& POISONING & $800-999$ & 2 & 0 & 9 & 6 & 17 & 34 \\
\hline -Fracture - Upper Limb & $810-819$ & 0 & 0 & 1 & 0 & 1 & 2 \\
\hline -Fracture - Lower Limb & $820-829$ & 0 & 0 & 1 & 0 & 1 & 3 \\
\hline -Dislocation & $830-839$ & 0 & 0 & 0 & 1 & 1 & 1 \\
\hline -Sprains \& Strains - Back & 846-847 & 0 & 0 & 0 & 0 & 0 & 2 \\
\hline -Sprains \& Strains - Other & $840-845,848$ & 0 & 0 & 3 & 2 & 5 & 6 \\
\hline -Open Wound - Head, Neck, Trunk & 870-879 & 0 & 0 & 1 & 0 & 1 & 1 \\
\hline -Open Wound - Upper Limb & $880-887$ & 1 & 0 & 0 & 2 & 3 & 4 \\
\hline -Open Wound - Lower Limb & $890-897$ & 1 & 0 & 1 & 0 & 2 & 3 \\
\hline -Superficial Injury & $910-919$ & 0 & 0 & 1 & 0 & 1 & 3 \\
\hline -Contusion & $920-924$ & 0 & 0 & 1 & 0 & 1 & 5 \\
\hline -Foreign Body Entering Orifice & $930-939$ & 0 & 0 & 0 & 1 & 1 & 2 \\
\hline -Toxic Effects - Non-medicinal & $980-989$ & 0 & 0 & 0 & 0 & 0 & 1 \\
\hline -Unspecified Effects - External Causes & $990-995$ & 0 & 0 & 0 & 0 & 0 & 1 \\
\hline
\end{tabular}

\begin{tabular}{|c|c|c|c|c|c|c|c|c|c|c|}
\hline & \multicolumn{4}{|c|}{ Women } & \multicolumn{5}{|c|}{ Men } & \multirow[b]{3}{*}{ TOTAL } \\
\hline & \multicolumn{3}{|c|}{ Age Group } & \multirow[b]{2}{*}{ TOTAL } & \multicolumn{4}{|c|}{ Age Group } & \multirow[b]{2}{*}{ TOTAL } & \\
\hline & $16-29$ & $40-49$ & $50+$ & & $16-29$ & $30-39$ & $40-49$ & $50+$ & & \\
\hline Diagnostic Category & & & & & & & & & & \\
\hline Total & 1 & 3 & 19 & 23 & 2 & 2 & 11 & 9 & 24 & 47 \\
\hline
\end{tabular}

*Only those diagnostic categories and gender/age combinations with at least one OSHA event appear in this table. 


\section{Savannah River Site 2008}

OSHA Data

Appendix P. Number of Workdays Lost or with Restricted Activity in Each Diagnostic Category by Gender and Age*

\begin{tabular}{|c|c|c|c|c|c|c|c|}
\hline & & \multicolumn{6}{|c|}{ Women } \\
\hline & & \multicolumn{6}{|c|}{ Age Group } \\
\hline & & \multicolumn{2}{|c|}{$16-29$} & \multicolumn{2}{|c|}{$40-49$} & \multicolumn{2}{|c|}{$50+$} \\
\hline & & $\begin{array}{c}\text { Days } \\
\text { Restricted }\end{array}$ & Days Lost & \begin{tabular}{c|} 
Days \\
Restricted
\end{tabular} & Days Lost & \begin{tabular}{|c|} 
Days \\
Restricted
\end{tabular} & Days Lost \\
\hline Diagnostic Category & ICD-9-CM Codes & & & & & & \\
\hline $\begin{array}{l}\text {-Other Metabolic \& Immunity } \\
\text { Disorders }\end{array}$ & $270-279$ & 0 & 0 & 0 & 0 & 0 & 0 \\
\hline -Arthropathies & $710-719$ & 0 & 0 & 0 & 0 & 16 & 0 \\
\hline -Dorsopathies & $720-724$ & 0 & 0 & 0 & 0 & 0 & 0 \\
\hline -Rheumatism, Excluding Back & $725-729$ & 0 & 0 & 0 & 0 & 16 & 0 \\
\hline -Symptoms & $780-789$ & 0 & 0 & 0 & 0 & 0 & 49 \\
\hline -Fracture - Upper Limb & $810-819$ & 0 & 0 & 126 & 0 & 0 & $\overline{0}$ \\
\hline -Fracture - Lower Limb & $820-829$ & 0 & 0 & 0 & 0 & 81 & 69 \\
\hline -Sprains \& Strains - Back & 846-847 & 0 & 0 & 0 & 0 & 16 & 0 \\
\hline -Sprains \& Strains - Other & $840-845,848$ & 0 & 0 & 0 & 0 & 8 & $\overline{0}$ \\
\hline -Open Wound - Upper Limb & $880-887$ & 0 & 0 & 0 & 0 & 0 & 0 \\
\hline -Open Wound - Lower Limb & $890-897$ & 0 & 0 & 0 & 0 & 0 & 0 \\
\hline -Superficial Injury & $910-919$ & 0 & 0 & 0 & 0 & 0 & $\overline{0}$ \\
\hline -Contusion & $920-924$ & 0 & 0 & 0 & 0 & 0 & 0 \\
\hline -Foreign Body Entering Orifice & 930-939 & 0 & 0 & 0 & 0 & 0 & 0 \\
\hline -Toxic Effects - Non-medicinal & $980-989$ & 0 & 0 & 0 & 0 & 0 & 0 \\
\hline $\begin{array}{l}\text {-Unspecified Effects - External } \\
\text { Causes }\end{array}$ & 990-995 & 0 & 0 & 0 & 0 & 0 & 0 \\
\hline
\end{tabular}

\begin{tabular}{|c|c|c|c|c|c|c|c|c|c|}
\hline & & \multicolumn{8}{|c|}{ Men } \\
\hline & & \multicolumn{8}{|c|}{ Age Group } \\
\hline & & \multicolumn{2}{|c|}{$16-29$} & \multicolumn{2}{|c|}{$30-39$} & \multicolumn{2}{|c|}{$40-49$} & \multicolumn{2}{|c|}{$50+$} \\
\hline & & $\begin{array}{c}\text { Days } \\
\text { Restricted }\end{array}$ & $\begin{array}{l}\text { Days } \\
\text { Lost }\end{array}$ & $\begin{array}{c}\text { Days } \\
\text { Restricted }\end{array}$ & $\begin{array}{l}\text { Days } \\
\text { Lost }\end{array}$ & \begin{tabular}{|c|} 
Days \\
Restricted
\end{tabular} & $\begin{array}{l}\text { Days } \\
\text { Lost }\end{array}$ & $\begin{array}{c}\text { Days } \\
\text { Restricted }\end{array}$ & $\begin{array}{l}\text { Days } \\
\text { Lost }\end{array}$ \\
\hline Diagnostic Category & ICD-9-CM Codes & \multirow[b]{2}{*}{0} & \multirow[b]{2}{*}{0} & \multirow[b]{2}{*}{0} & \multirow[b]{2}{*}{0} & \multirow[b]{2}{*}{0} & \multirow[b]{2}{*}{0} & \multirow[b]{2}{*}{0} & \multirow[b]{2}{*}{0} \\
\hline -Lung Dis from External Agents & $500-508$ & & & & & & & & \\
\hline -Arthropathies & $710-719$ & 0 & 0 & 0 & 0 & 0 & 0 & 0 & 0 \\
\hline -Dorsopathies & $720-724$ & 0 & 0 & 22 & 0 & 0 & 0 & 0 & 0 \\
\hline -Rheumatism, Excluding Back & 725-729 & 0 & 0 & 22 & 0 & 0 & 0 & 15 & 0 \\
\hline -Fracture - Upper Limb & $810-819$ & 0 & 0 & 0 & 0 & 104 & 0 & 0 & 0 \\
\hline -Fracture - Lower Limb & $820-829$ & 0 & 0 & 0 & 0 & 0 & 0 & 0 & 0 \\
\hline -Dislocation & $830-839$ & 0 & 0 & 0 & 0 & 0 & 0 & 0 & 0 \\
\hline -Sprains \& Strains - Other & $840-845,848$ & 0 & 0 & 0 & 0 & 98 & 0 & 32 & 28 \\
\hline -Open Wound - Head, Neck, Trunk & 870-879 & 0 & 0 & 0 & 0 & 0 & 0 & 0 & 0 \\
\hline -Open Wound - Upper Limb & 880-887 & 0 & 0 & 0 & 0 & 0 & 0 & 0 & 0 \\
\hline -Open Wound - Lower Limb & 890-897 & 0 & 0 & 0 & 0 & \begin{tabular}{l|l}
0 \\
\end{tabular} & 0 & 0 & 0 \\
\hline
\end{tabular}

(Continued)

*OSHA events with >1 ICD-9-CM code in the same diagnostic category were counted only once. Only those diagnostic categories and gender/age combinations with at least one occurrence appear in this table. 


\section{Savannah River Site 2008}

OSHA Data

Appendix P. Number of Workdays Lost or with Restricted Activity in Each Diagnostic Category by Gender and Age*

\begin{tabular}{|c|c|c|c|c|c|c|c|c|c|}
\hline & & \multicolumn{8}{|c|}{ Men } \\
\hline & & \multicolumn{8}{|c|}{ Age Group } \\
\hline & & \multicolumn{2}{|c|}{$16-29$} & \multicolumn{2}{|c|}{30 - 39} & \multicolumn{2}{|c|}{$40-49$} & \multicolumn{2}{|c|}{$50+$} \\
\hline & & $\begin{array}{c}\text { Days } \\
\text { Restricted }\end{array}$ & $\begin{array}{l}\text { Days } \\
\text { Lost }\end{array}$ & $\begin{array}{c}\text { Days } \\
\text { Restricted }\end{array}$ & $\begin{array}{l}\text { Days } \\
\text { Lost }\end{array}$ & $\begin{array}{c}\text { Days } \\
\text { Restricted }\end{array}$ & $\begin{array}{l}\text { Days } \\
\text { Lost }\end{array}$ & $\begin{array}{c}\text { Days } \\
\text { Restricted }\end{array}$ & $\begin{array}{l}\text { Days } \\
\text { Lost }\end{array}$ \\
\hline Diagnostic Category & ICD-9-CM Codes & & & & & & & & \\
\hline -Superficial Injury & 910-919 & 0 & 0 & 0 & 0 & 0 & 0 & 0 & 0 \\
\hline -Contusion & $920-924$ & 0 & 0 & 0 & 0 & 0 & 0 & 0 & 0 \\
\hline -Foreign Body Entering Orifice & 930-939 & 0 & 0 & 0 & 0 & 0 & 0 & 0 & 0 \\
\hline
\end{tabular}

*OSHA events with >1 ICD-9-CM code in the same diagnostic category were counted only once. Only those diagnostic categories and gender/age combinations with at least one occurrence appear in this table. 
Savannah River Site 2008

OSHA Data

Appendix Q. Number of Occurrences in Each Accident Category by Gender and Age*

\begin{tabular}{|c|c|c|c|c|c|c|c|c|c|c|c|}
\hline & & \multicolumn{4}{|c|}{ Women } & \multicolumn{5}{|c|}{ Men } & \multirow[b]{3}{*}{ TOTAL } \\
\hline & & \multicolumn{3}{|c|}{ Age Group } & \multirow[b]{2}{*}{ TOTAL } & \multicolumn{4}{|c|}{ Age Group } & \multirow[b]{2}{*}{ TOTAL } & \\
\hline & & 16 - 29 & 40 - 49 & $\begin{array}{l}50 \\
+\end{array}$ & & $16-29$ & 30 - 39 & $40-49$ & $\begin{array}{l}50 \\
+\end{array}$ & & \\
\hline Type of Accident & E Codes & & & & & & & & & & \\
\hline Accidental Poisoning - Non-medicinal & E860-E869 & 0 & 1 & 0 & 1 & 0 & 0 & 0 & 0 & 0 & 1 \\
\hline Falls & E880-E888 & 0 & 1 & 4 & 5 & 0 & 0 & 3 & 2 & 5 & 10 \\
\hline Natural/Environmental Factors & E900-E909 & 0 & 0 & 1 & 1 & 0 & 0 & 0 & 0 & 0 & 1 \\
\hline $\begin{array}{l}\text { Submersion/Suffocation/Foreign } \\
\text { Bodies }\end{array}$ & E910-E915 & 0 & 1 & 0 & 1 & 0 & 0 & 0 & 1 & 1 & 2 \\
\hline Other Accidents & E916-E928 & 1 & 0 & 4 & 5 & 2 & 1 & 5 & 4 & 12 & 17 \\
\hline
\end{tabular}

*Only those accident types and gender/age combinations with at least one occurrence appear in this table. 


\section{Savannah River Site 2008}

OSHA Data

Appendix R. Number of Workdays Lost or with Restricted Activity in Each Accident Category by Gender and Age*

\begin{tabular}{|c|c|c|c|c|c|c|c|}
\hline & & \multicolumn{6}{|c|}{ Women } \\
\hline & & \multicolumn{6}{|c|}{ Age Group } \\
\hline & & \multicolumn{2}{|c|}{$16-29$} & \multicolumn{2}{|c|}{$40-49$} & \multicolumn{2}{|c|}{$50+$} \\
\hline & & \begin{tabular}{c|} 
Days \\
Restricted
\end{tabular} & Days Lost & $\begin{array}{c}\text { Days } \\
\text { Restricted }\end{array}$ & Days Lost & $\begin{array}{c}\text { Days } \\
\text { Restricted }\end{array}$ & Days Lost \\
\hline Type of Accident & E Codes & \multirow[b]{2}{*}{0} & \multirow[b]{2}{*}{0} & \multirow[b]{2}{*}{0} & \multirow[b]{2}{*}{0} & \multirow[b]{2}{*}{0} & \multirow[b]{2}{*}{0} \\
\hline Accidental Poisoning - Non-medicinal & E860-E869 & & & & & & \\
\hline Falls & E880-E888 & 0 & 0 & 126 & 0 & 97 & 118 \\
\hline Natural/Environmental Factors & E900-E909 & 0 & 0 & 0 & 0 & 0 & 0 \\
\hline $\begin{array}{l}\text { Submersion/Suffocation/Foreign } \\
\text { Bodies }\end{array}$ & E910-E915 & 0 & 0 & 0 & 0 & 0 & 0 \\
\hline Other Accidents & E916-E928 & 0 & 0 & 0 & 0 & 8 & 0 \\
\hline
\end{tabular}

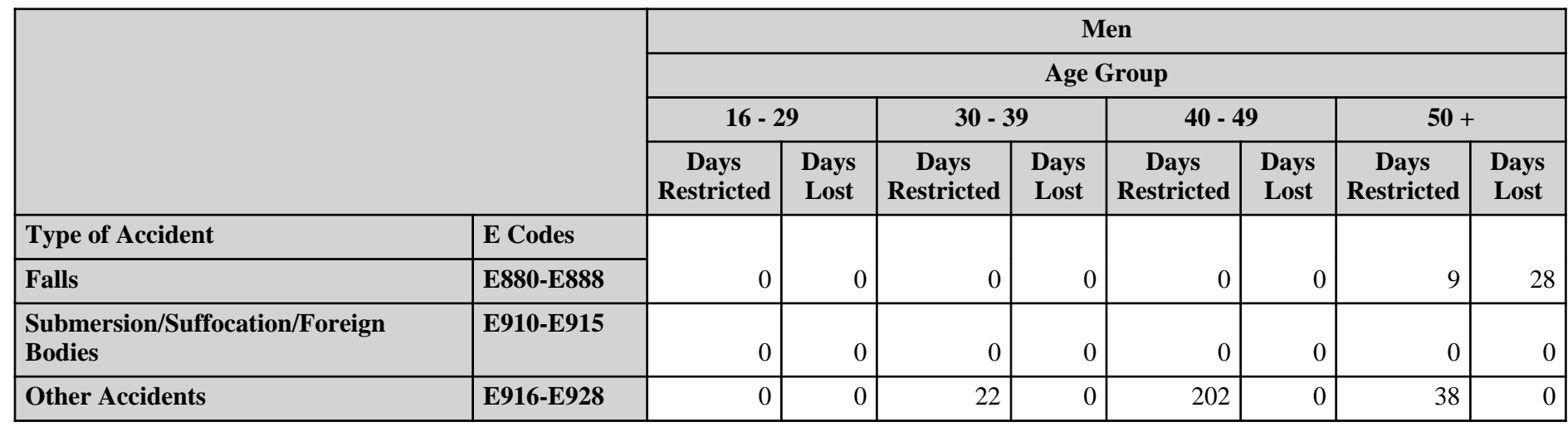

*OSHA events with $>1 \mathrm{E}$ code in the same accident type were counted only once. Only those accident types and gender/age combinations with at least one occurrence appear in this table. 


\section{Savannah River Site 2008}

\section{OSHA Data}

Appendix S. Number of Diagnoses in Each Diagnostic Category by Gender and Job Category*

\begin{tabular}{|c|c|c|c|c|c|c|}
\hline & & \multicolumn{5}{|c|}{ Women } \\
\hline & & \multicolumn{4}{|c|}{ Job Category } & \multirow[b]{2}{*}{ TOTAL } \\
\hline & & Professional & $\begin{array}{c}\text { Administrative } \\
\text { Support }\end{array}$ & Technical Support & Line Operators & \\
\hline Diagnostic Category & ICD-9-CM Code & & & & & \\
\hline $\begin{array}{l}\text { ENDOCRINE/ } \\
\text { METABOLIC/ } \\
\text { IMMUNITY }\end{array}$ & 240-279 & 0 & 0 & 1 & 0 & 1 \\
\hline $\begin{array}{l}\text {-Other } \\
\text { Metabolic \& Immunity } \\
\text { Disorders }\end{array}$ & 270-279 & 0 & 0 & 1 & 0 & 1 \\
\hline $\begin{array}{l}\text { MUSCULOSKELETAL } \\
\text { \& CONNECTIVE } \\
\text { TISSUE }\end{array}$ & $710-739$ & 0 & 0 & 1 & 3 & 4 \\
\hline -Arthropathies & $710-719$ & 0 & 0 & 0 & 2 & 2 \\
\hline -Dorsopathies & $720-724$ & 0 & 0 & 1 & 0 & 1 \\
\hline $\begin{array}{l}\text {-Rheumatism, Excluding } \\
\text { Back }\end{array}$ & 725-729 & 0 & 0 & 0 & 1 & 1 \\
\hline $\begin{array}{l}\text { SYMPTOMS, } \\
\text { SIGNS, \& ILL-DEFINED } \\
\text { CONDITIONS }\end{array}$ & 780-799 & 1 & 0 & 0 & 0 & 1 \\
\hline -Symptoms & 780-789 & 1 & 0 & 0 & 0 & 1 \\
\hline INJURY \& POISONING & 800-999 & 0 & 5 & 7 & 5 & 17 \\
\hline -Fracture - Upper Limb & $810-819$ & 0 & 0 & 0 & 1 & 1 \\
\hline -Fracture - Lower Limb & $820-829$ & 0 & 2 & 0 & 0 & 2 \\
\hline -Sprains \& Strains - Back & 846-847 & 0 & 0 & 1 & 1 & 2 \\
\hline $\begin{array}{l}\text {-Sprains \& Strains - } \\
\text { Other }\end{array}$ & $840-845,848$ & 0 & 1 & 0 & 0 & 1 \\
\hline $\begin{array}{l}\text {-Open Wound - Upper } \\
\text { Limb }\end{array}$ & $880-887$ & 0 & 0 & 0 & 1 & 1 \\
\hline $\begin{array}{l}\text {-Open Wound - Lower } \\
\text { Limb }\end{array}$ & 890-897 & 0 & 1 & 0 & 0 & 1 \\
\hline -Superficial Injury & 910-919 & 0 & 0 & 2 & 0 & 2 \\
\hline -Contusion & 920-924 & 0 & 1 & 2 & 1 & 4 \\
\hline $\begin{array}{l}\text {-Foreign Body Entering } \\
\text { Orifice }\end{array}$ & 930-939 & 0 & 0 & 1 & 0 & 1 \\
\hline $\begin{array}{l}\text {-Toxic } \\
\text { Effects - Non-medicinal }\end{array}$ & 980-989 & 0 & 0 & 0 & 1 & 1 \\
\hline $\begin{array}{l}\text {-Unspecified } \\
\text { Effects - External Causes }\end{array}$ & $990-995$ & 0 & 0 & 1 & 0 & 1 \\
\hline
\end{tabular}

*Only those diagnostic categories and gender/job category combinations with at least one occurrence appear in this table. 


\section{Savannah River Site 2008}

\section{OSHA Data}

Appendix S. Number of Diagnoses in Each Diagnostic Category by Gender and Job Category*

\begin{tabular}{|c|c|c|c|c|c|}
\hline & \multicolumn{5}{|c|}{ Women } \\
\hline & \multicolumn{4}{|c|}{ Job Category } & \multirow[b]{2}{*}{ TOTAL } \\
\hline & Professional & $\begin{array}{c}\text { Administrative } \\
\text { Support }\end{array}$ & Technical Support & Line Operators & \\
\hline Diagnostic Category & \multirow[b]{2}{*}{1} & \multirow[b]{2}{*}{5} & \multirow[b]{2}{*}{9} & \multirow[b]{2}{*}{8} & \multirow[b]{2}{*}{23} \\
\hline Total & & & & & \\
\hline
\end{tabular}

\begin{tabular}{|c|c|c|c|c|c|c|c|c|}
\hline & & \multicolumn{7}{|c|}{ Men } \\
\hline & & \multicolumn{6}{|c|}{ Job Category } & \multirow[b]{2}{*}{ TOTAL } \\
\hline & & Professional & $\begin{array}{c}\text { Administrative } \\
\text { Support }\end{array}$ & $\begin{array}{l}\text { Technical } \\
\text { Support }\end{array}$ & Service & Crafts & $\begin{array}{c}\text { Line } \\
\text { Operators }\end{array}$ & \\
\hline Diagnostic Category & ICD-9-CM Code & & & & & & & \\
\hline $\begin{array}{l}\text { RESPIRATORY } \\
\text { SYSTEM }\end{array}$ & $460-519$ & 0 & 1 & 0 & 0 & 0 & 1 & 2 \\
\hline $\begin{array}{l}\text {-Lung Dis from External } \\
\text { Agents }\end{array}$ & $500-508$ & 0 & 1 & 0 & 0 & 0 & 1 & 2 \\
\hline $\begin{array}{l}\text { MUSCULOSKELETAL } \\
\text { \& CONNECTIVE } \\
\text { TISSUE }\end{array}$ & $710-739$ & 0 & 0 & 3 & 0 & 1 & 1 & 5 \\
\hline -Arthropathies & $710-719$ & 0 & 0 & 1 & 0 & 0 & 0 & 1 \\
\hline -Dorsopathies & $720-724$ & 0 & 0 & 1 & 0 & 0 & 0 & 1 \\
\hline $\begin{array}{l}\text {-Rheumatism, Excluding } \\
\text { Back }\end{array}$ & $725-729$ & 0 & 0 & 1 & 0 & 1 & 1 & 3 \\
\hline INJURY \& POISONING & $800-999$ & 2 & 1 & 2 & 1 & 7 & 4 & 17 \\
\hline -Fracture - Upper Limb & $810-819$ & 0 & 0 & 0 & 0 & 1 & 0 & 1 \\
\hline -Fracture - Lower Limb & $820-829$ & 1 & 0 & 0 & 0 & 0 & 0 & 1 \\
\hline -Dislocation & $830-839$ & 0 & 1 & 0 & 0 & 0 & 0 & 1 \\
\hline $\begin{array}{l}\text {-Sprains \& Strains - } \\
\text { Other }\end{array}$ & $840-845,848$ & 0 & 0 & 2 & 0 & 1 & 2 & 5 \\
\hline $\begin{array}{l}\text {-Open Wound - Head, } \\
\text { Neck, Trunk }\end{array}$ & $870-879$ & 1 & 0 & 0 & 0 & 0 & 0 & 1 \\
\hline $\begin{array}{l}\text {-Open Wound - Upper } \\
\text { Limb }\end{array}$ & $880-887$ & 0 & 0 & 0 & 1 & 2 & 0 & 3 \\
\hline $\begin{array}{l}\text {-Open Wound - Lower } \\
\text { Limb }\end{array}$ & $890-897$ & 0 & 0 & 0 & 0 & 1 & 1 & 2 \\
\hline -Superficial Injury & 910-919 & 0 & 0 & 0 & 0 & 1 & 0 & 1 \\
\hline -Contusion & \begin{tabular}{|l|}
$920-924$ \\
\end{tabular} & 0 & 0 & 0 & 0 & 1 & 0 & 1 \\
\hline $\begin{array}{l}\text {-Foreign Body Entering } \\
\text { Orifice }\end{array}$ & 930-939 & 0 & 0 & 0 & 0 & 0 & 1 & 1 \\
\hline
\end{tabular}

*Only those diagnostic categories and gender/job category combinations with at least one occurrence appear in this table. 
Savannah River Site 2008

OSHA Data

Appendix S. Number of Diagnoses in Each Diagnostic Category by Gender and Job Category*

\begin{tabular}{|c|c|c|c|c|c|c|c|}
\hline & \multicolumn{7}{|c|}{ Men } \\
\hline & \multicolumn{6}{|c|}{ Job Category } & \multirow[b]{2}{*}{ TOTAL } \\
\hline & Professional & $\begin{array}{c}\text { Administrative } \\
\text { Support }\end{array}$ & Technical Support & Service & Crafts & Line Operators & \\
\hline Diagnostic Category & \multirow[b]{2}{*}{2} & \multirow[b]{2}{*}{2} & \multirow[b]{2}{*}{5} & \multirow[b]{2}{*}{1} & \multirow[b]{2}{*}{8} & \multirow[b]{2}{*}{6} & \multirow[b]{2}{*}{24} \\
\hline Total & & & & & & & \\
\hline
\end{tabular}

*Only those diagnostic categories and gender/job category combinations with at least one occurrence appear in this table. 


\section{Savannah River Site 2008}

OSHA Data

Appendix T. Number of Workdays Lost or with Restricted Activity in Each Diagnostic Category by Gender and Job Category*

\begin{tabular}{|c|c|c|c|c|c|c|c|c|c|}
\hline & & \multicolumn{8}{|c|}{ Women } \\
\hline & & \multicolumn{8}{|c|}{ Job Category } \\
\hline & & \multicolumn{2}{|c|}{ Professional } & \multicolumn{2}{|c|}{$\begin{array}{l}\text { Administrative } \\
\text { Support }\end{array}$} & \multicolumn{2}{|c|}{$\begin{array}{l}\text { Technical } \\
\text { Support }\end{array}$} & \multicolumn{2}{|c|}{ Line Operators } \\
\hline & & $\begin{array}{c}\text { Days } \\
\text { Restricted }\end{array}$ & $\begin{array}{l}\text { Days } \\
\text { Lost }\end{array}$ & \begin{tabular}{c|} 
Days \\
Restricted
\end{tabular} & $\begin{array}{l}\text { Days } \\
\text { Lost }\end{array}$ & \begin{tabular}{|c|} 
Days \\
Restricted
\end{tabular} & $\begin{array}{l}\text { Days } \\
\text { Lost }\end{array}$ & \begin{tabular}{c|} 
Days \\
Restricted
\end{tabular} & $\begin{array}{l}\text { Days } \\
\text { Lost }\end{array}$ \\
\hline Diagnostic Category & ICD-9-CM Codes & & & & & & & & \\
\hline $\begin{array}{l}\text {-Other Metabolic \& Immunity } \\
\text { Disorders }\end{array}$ & $270-279$ & 0 & 0 & 0 & 0 & 0 & 0 & 0 & 0 \\
\hline -Arthropathies & $710-719$ & 0 & 0 & 0 & 0 & 0 & 0 & 16 & 0 \\
\hline -Dorsopathies & $720-724$ & 0 & 0 & 0 & 0 & 0 & 0 & 0 & 0 \\
\hline -Rheumatism, Excluding Back & $725-729$ & 0 & 0 & 0 & 0 & 0 & 0 & 16 & 0 \\
\hline -Symptoms & \begin{tabular}{|l|}
$780-789$ \\
\end{tabular} & 0 & 49 & 0 & 0 & 0 & 0 & 0 & 0 \\
\hline -Fracture - Upper Limb & $810-819$ & 0 & 0 & 0 & 0 & 0 & 0 & 126 & 0 \\
\hline -Fracture - Lower Limb & $820-829$ & 0 & 0 & 81 & 69 & 0 & 0 & 0 & 0 \\
\hline -Sprains \& Strains - Back & 846-847 & 0 & 0 & 0 & 0 & 0 & 0 & 16 & 0 \\
\hline -Sprains \& Strains - Other & $840-845,848$ & 0 & 0 & 8 & 0 & 0 & 0 & 0 & 0 \\
\hline -Open Wound - Upper Limb & $880-887$ & 0 & 0 & 0 & 0 & 0 & 0 & 0 & 0 \\
\hline -Open Wound - Lower Limb & $890-897$ & 0 & 0 & 0 & 0 & 0 & 0 & 0 & 0 \\
\hline -Superficial Injury & $910-919$ & 0 & 0 & 0 & 0 & 0 & 0 & 0 & 0 \\
\hline -Contusion & $920-924$ & 0 & 0 & 0 & 0 & 0 & 0 & 0 & 0 \\
\hline -Foreign Body Entering Orifice & 930-939 & 0 & 0 & 0 & 0 & 0 & 0 & 0 & 0 \\
\hline -Toxic Effects - Non-medicinal & 980-989 & 0 & 0 & 0 & 0 & 0 & 0 & 0 & 0 \\
\hline $\begin{array}{l}\text {-Unspecified Effects - External } \\
\text { Causes }\end{array}$ & \begin{tabular}{|c|}
$990-995$ \\
\end{tabular} & 0 & 0 & 0 & 0 & 0 & 0 & 0 & 0 \\
\hline
\end{tabular}

*OSHA events with >1 ICD-9-CM code in the same diagnostic category were counted only once. Only those diagnostic categories and gender/job category combinations with at least one occurrence appear in this table. 


\section{Savannah River Site 2008}

OSHA Data

Appendix T. Number of Workdays Lost or with Restricted Activity in Each Diagnostic Category by Gender and Job Category*

\begin{tabular}{|c|c|c|c|c|c|c|c|}
\hline & & \multicolumn{6}{|c|}{ Men } \\
\hline & & \multicolumn{6}{|c|}{ Job Category } \\
\hline & & \multicolumn{2}{|c|}{ Professional } & \multicolumn{2}{|c|}{$\begin{array}{c}\text { Administrative } \\
\text { Support }\end{array}$} & \multicolumn{2}{|c|}{ Technical Support } \\
\hline & & $\begin{array}{c}\text { Days } \\
\text { Restricted }\end{array}$ & Days Lost & \begin{tabular}{|c|} 
Days \\
Restricted
\end{tabular} & Days Lost & \begin{tabular}{|c|} 
Days \\
Restricted
\end{tabular} & Days Lost \\
\hline Diagnostic Category & ICD-9-CM Codes & \multirow[b]{2}{*}{0} & \multirow[b]{2}{*}{0} & \multirow[b]{2}{*}{0} & \multirow[b]{2}{*}{0} & \multirow[b]{2}{*}{0} & \multirow[b]{2}{*}{0} \\
\hline -Lung Dis from External Agents & $500-508$ & & & & & & \\
\hline -Arthropathies & $710-719$ & 0 & 0 & 0 & 0 & 0 & 0 \\
\hline -Dorsopathies & $720-724$ & 0 & 0 & 0 & 0 & 22 & 0 \\
\hline -Rheumatism, Excluding Back & $725-729$ & 0 & 0 & 0 & 0 & 22 & 0 \\
\hline -Fracture - Upper Limb & $810-819$ & 0 & 0 & 0 & 0 & 0 & 0 \\
\hline -Fracture - Lower Limb & \begin{tabular}{|c|}
$820-829$ \\
\end{tabular} & 0 & 0 & 0 & 0 & 0 & 0 \\
\hline -Dislocation & $830-839$ & 0 & 0 & 0 & 0 & 0 & 0 \\
\hline -Sprains \& Strains - Other & $840-845,848$ & 0 & 0 & 0 & 0 & 9 & 28 \\
\hline -Open Wound - Head, Neck, Trunk & $870-879$ & 0 & 0 & 0 & 0 & 0 & 0 \\
\hline -Open Wound - Upper Limb & \begin{tabular}{|c|}
$880-887$ \\
\end{tabular} & 0 & 0 & 0 & 0 & 0 & 0 \\
\hline -Open Wound - Lower Limb & 890-897 & 0 & 0 & 0 & 0 & 0 & 0 \\
\hline -Superficial Injury & 910-919 & 0 & 0 & 0 & 0 & 0 & 0 \\
\hline -Contusion & $920-924$ & 0 & 0 & 0 & 0 & 0 & 0 \\
\hline -Foreign Body Entering Orifice & 930-939 & 0 & 0 & 0 & 0 & 0 & 0 \\
\hline
\end{tabular}

*OSHA events with >1 ICD-9-CM code in the same diagnostic category were counted only once. Only those diagnostic categories and gender/job category combinations with at least one occurrence appear in this table. 


\section{Savannah River Site 2008}

OSHA Data

Appendix T. Number of Workdays Lost or with Restricted Activity in Each Diagnostic Category by Gender and Job Category*

\begin{tabular}{|c|c|c|c|c|c|c|c|}
\hline & & \multicolumn{6}{|c|}{ Men } \\
\hline & & \multicolumn{6}{|c|}{ Job Category } \\
\hline & & \multicolumn{2}{|c|}{ Service } & \multicolumn{2}{|c|}{ Crafts } & \multicolumn{2}{|c|}{ Line Operators } \\
\hline & & $\begin{array}{c}\text { Days } \\
\text { Restricted }\end{array}$ & Days Lost & \begin{tabular}{|c|} 
Days \\
Restricted
\end{tabular} & Days Lost & \begin{tabular}{|c|} 
Days \\
Restricted
\end{tabular} & Days Lost \\
\hline Diagnostic Category & ICD-9-CM Codes & \multirow[b]{2}{*}{0} & \multirow[b]{2}{*}{0} & \multirow[b]{2}{*}{0} & \multirow[b]{2}{*}{0} & \multirow[b]{2}{*}{0} & \multirow[b]{2}{*}{0} \\
\hline -Lung Dis from External Agents & $500-508$ & & & & & & \\
\hline -Arthropathies & $710-719$ & 0 & 0 & 0 & 0 & 0 & 0 \\
\hline -Dorsopathies & $720-724$ & 0 & 0 & 0 & 0 & 0 & 0 \\
\hline -Rheumatism, Excluding Back & $725-729$ & 0 & 0 & 0 & 0 & 15 & 0 \\
\hline -Fracture - Upper Limb & $810-819$ & 0 & 0 & 104 & 0 & 0 & 0 \\
\hline -Fracture - Lower Limb & \begin{tabular}{|c|}
$820-829$ \\
\end{tabular} & 0 & 0 & 0 & 0 & 0 & 0 \\
\hline -Dislocation & $830-839$ & 0 & 0 & 0 & 0 & 0 & 0 \\
\hline -Sprains \& Strains - Other & $840-845,848$ & 0 & 0 & 42 & 0 & 79 & 0 \\
\hline -Open Wound - Head, Neck, Trunk & $870-879$ & 0 & 0 & 0 & 0 & 0 & 0 \\
\hline -Open Wound - Upper Limb & \begin{tabular}{|c|}
$880-887$ \\
\end{tabular} & 0 & 0 & 0 & 0 & 0 & 0 \\
\hline -Open Wound - Lower Limb & 890-897 & 0 & 0 & 0 & 0 & 0 & 0 \\
\hline -Superficial Injury & 910-919 & 0 & 0 & 0 & 0 & 0 & 0 \\
\hline -Contusion & $920-924$ & 0 & 0 & 0 & 0 & 0 & 0 \\
\hline -Foreign Body Entering Orifice & 930-939 & 0 & 0 & 0 & 0 & 0 & 0 \\
\hline
\end{tabular}

*OSHA events with >1 ICD-9-CM code in the same diagnostic category were counted only once. Only those diagnostic categories and gender/job category combinations with at least one occurrence appear in this table. 


\section{Savannah River Site 2008}

OSHA Data

Appendix U. Number of Occurrences in Each Accident Category by Gender and Job Category*

\begin{tabular}{|c|c|c|c|c|c|c|}
\hline & & \multicolumn{5}{|c|}{ Women } \\
\hline & & \multicolumn{4}{|c|}{ Job Category } & \multirow[b]{2}{*}{ TOTAL } \\
\hline & & Professional & $\begin{array}{c}\text { Administrative } \\
\text { Support }\end{array}$ & $\begin{array}{l}\text { Technical } \\
\text { Support }\end{array}$ & \begin{tabular}{|c|} 
Line \\
Operators
\end{tabular} & \\
\hline Type of Accident & E CODES & \multirow[b]{2}{*}{0} & \multirow[b]{2}{*}{0} & \multirow[b]{2}{*}{0} & \multirow[b]{2}{*}{1} & \multirow[b]{2}{*}{1} \\
\hline Accidental Poisoning - Non-medicinal & E860-E869 & & & & & \\
\hline Falls & E880-E888 & 1 & 1 & 1 & 2 & 5 \\
\hline Natural/Environmental Factors & E900-E909 & 0 & 0 & 1 & 0 & 1 \\
\hline $\begin{array}{l}\text { Submersion/Suffocation/Foreign } \\
\text { Bodies }\end{array}$ & E910-E915 & 0 & 0 & 1 & 0 & 1 \\
\hline Other Accidents & E916-E928 & 0 & 2 & 1 & 2 & 5 \\
\hline
\end{tabular}

\begin{tabular}{|c|c|c|c|c|c|c|c|c|}
\hline & & \multicolumn{7}{|c|}{ Men } \\
\hline & & \multicolumn{6}{|c|}{ Job Category } & \multirow[b]{2}{*}{ TOTAL } \\
\hline & & Professional & $\begin{array}{c}\text { Administrative } \\
\text { Support }\end{array}$ & $\begin{array}{l}\text { Technical } \\
\text { Support }\end{array}$ & Service & Crafts & $\begin{array}{c}\text { Line } \\
\text { Operators }\end{array}$ & \\
\hline Type of Accident & E CODES & \multirow[b]{2}{*}{1} & \multirow[b]{2}{*}{1} & \multirow[b]{2}{*}{1} & \multirow[b]{2}{*}{0} & \multirow[b]{2}{*}{1} & \multirow[b]{2}{*}{1} & \multirow[b]{2}{*}{5} \\
\hline Falls & E880-E888 & & & & & & & \\
\hline $\begin{array}{l}\text { Submersion/Suffocation/Foreign } \\
\text { Bodies }\end{array}$ & E910-E915 & 0 & 0 & 0 & 0 & 0 & 1 & 1 \\
\hline Other Accidents & E916-E928 & 1 & 0 & 2 & 1 & 5 & 3 & 12 \\
\hline
\end{tabular}

*Only those accident types and gender/job category combinations with at least one occurrence appear in this table. 


\section{Savannah River Site 2008}

OSHA Data

Appendix V. Number of Workdays Lost or with Restricted Activity in Each Accident Category by Gender and Job Category*

\begin{tabular}{|c|c|c|c|c|c|c|c|c|c|}
\hline & & \multicolumn{8}{|c|}{ Women } \\
\hline & & \multicolumn{8}{|c|}{ Job Category } \\
\hline & & \multicolumn{2}{|c|}{ Professional } & \multicolumn{2}{|c|}{$\begin{array}{l}\text { Administrative } \\
\text { Support }\end{array}$} & \multicolumn{2}{|c|}{$\begin{array}{l}\text { Technical } \\
\text { Support }\end{array}$} & \multicolumn{2}{|c|}{ Line Operators } \\
\hline & & $\begin{array}{c}\text { Days } \\
\text { Restricted }\end{array}$ & $\begin{array}{l}\text { Days } \\
\text { Lost }\end{array}$ & $\begin{array}{c}\text { Days } \\
\text { Restricted }\end{array}$ & $\begin{array}{l}\text { Days } \\
\text { Lost }\end{array}$ & $\begin{array}{c}\text { Days } \\
\text { Restricted }\end{array}$ & $\begin{array}{c}\text { Days } \\
\text { Lost }\end{array}$ & \begin{tabular}{|c|} 
Days \\
Restricted
\end{tabular} & $\begin{array}{l}\text { Days } \\
\text { Lost }\end{array}$ \\
\hline Type of Accident & E Codes & & & & & & & & \\
\hline Accidental Poisoning - Non-medicinal & E860-E869 & 0 & 0 & 0 & 0 & 0 & 0 & 0 & 0 \\
\hline Falls & E880-E888 & 0 & 49 & 81 & 69 & 0 & 0 & 142 & 0 \\
\hline Natural/Environmental Factors & E900-E909 & 0 & 0 & 0 & 0 & 0 & 0 & 0 & 0 \\
\hline $\begin{array}{l}\text { Submersion/Suffocation/Foreign } \\
\text { Bodies }\end{array}$ & E910-E915 & 0 & 0 & 0 & 0 & 0 & 0 & 0 & 0 \\
\hline Other Accidents & E916-E928 & 0 & 0 & 8 & 0 & 0 & 0 & 0 & 0 \\
\hline
\end{tabular}

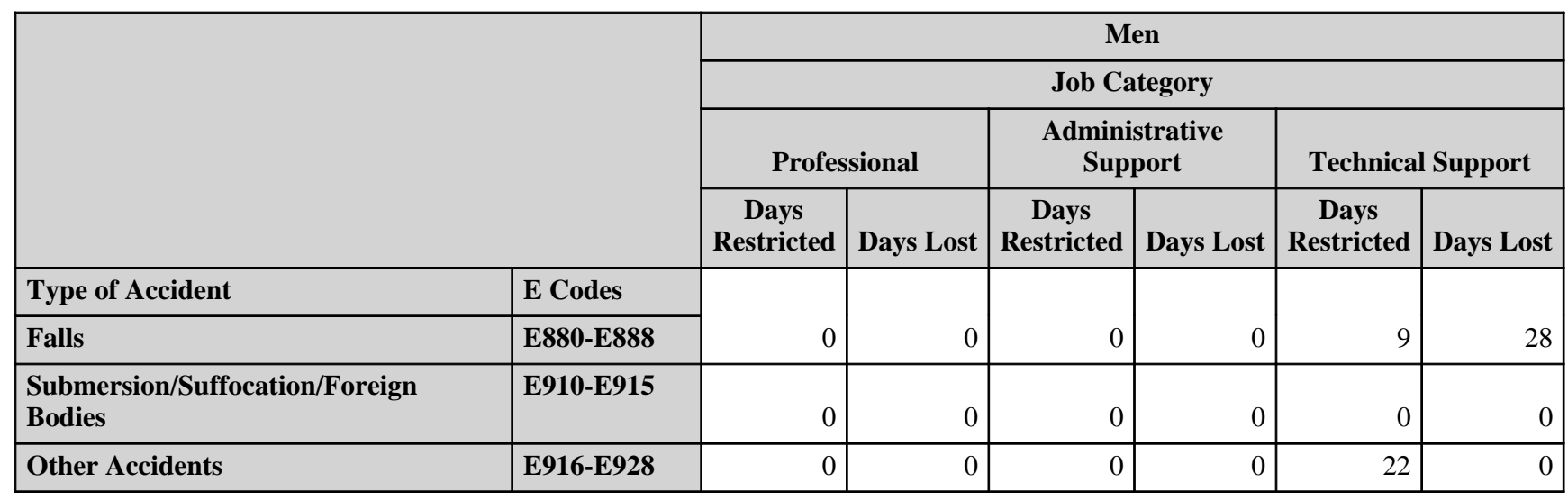

\begin{tabular}{|c|c|c|c|c|c|c|c|}
\hline & \multicolumn{6}{|c|}{ Men } \\
\hline & & \multicolumn{6}{|c|}{ Job Category } \\
\hline & & \multicolumn{2}{|c|}{ Service } & \multicolumn{2}{|c|}{ Crafts } & \multicolumn{2}{|c|}{ Line Operators } \\
\hline & & \begin{tabular}{|c|} 
Days \\
Restricted
\end{tabular} & Days Lost & \begin{tabular}{|c|} 
Days \\
Restricted
\end{tabular} & Days Lost & \begin{tabular}{|c|} 
Days \\
Restricted
\end{tabular} & Days Lost \\
\hline Type of Accident & E Codes & & & & & & \\
\hline Falls & E880-E888 & 0 & 0 & 0 & 0 & 0 & 0 \\
\hline $\begin{array}{l}\text { Submersion/Suffocation/Foreign } \\
\text { Bodies }\end{array}$ & E910-E915 & 0 & 0 & 0 & 0 & 0 & 0 \\
\hline Other Accidents & E916-E928 & 0 & 0 & 146 & 0 & 94 & 0 \\
\hline
\end{tabular}

*OSHA events with >1 E code in the same accident type were counted only once. Only those accident types and gender/job category combinations with at least one occurrence appear in this table. 
Savannah River Site 2008

OSHA Data

Appendix W. Age-Adjusted OSHA Illness and Injury Rates by Diagnostic Category*

Part 1. Men

\begin{tabular}{|c|c|c|c|c|c|}
\hline & & $\begin{array}{l}\text { Number of } \\
\text { Diagnoses }\end{array}$ & $\begin{array}{c}\text { Age-Adjusted } \\
\text { Rate per } 1,000^{* *}\end{array}$ & $\begin{array}{c}\text { Lower } 95 \% \\
\text { Confidence } \\
\text { Limit per 1,000 }\end{array}$ & $\begin{array}{c}\text { Upper } 95 \% \\
\text { Confidence } \\
\text { Limit per 1,000 }\end{array}$ \\
\hline Diagnostic Category & ICD-9-CM Code & \multirow[b]{2}{*}{2} & \multirow[b]{2}{*}{0.2} & \multirow[b]{2}{*}{0.0} & \multirow[b]{2}{*}{0.8} \\
\hline RESPIRATORY SYSTEM & $460-519$ & & & & \\
\hline -Lung Dis from External Agents & $500-508$ & 2 & 0.2 & 0.0 & 0.8 \\
\hline $\begin{array}{l}\text { MUSCULOSKELETAL \& CONNECTIVE } \\
\text { TISSUE }\end{array}$ & $710-739$ & 5 & 1.4 & 0.4 & 4.4 \\
\hline -Arthropathies & $710-719$ & 1 & 0.1 & 0.0 & 0.6 \\
\hline -Dorsopathies & $720-724$ & 1 & 0.6 & 0.1 & 4.0 \\
\hline -Rheumatism, Excluding Back & 725-729 & 3 & 0.7 & 0.1 & 3.5 \\
\hline INJURY \& POISONING & $800-999$ & 17 & 2.8 & 1.2 & 6.6 \\
\hline -Fracture - Upper Limb & $810-819$ & 1 & 0.1 & 0.0 & 0.6 \\
\hline -Fracture - Lower Limb & $820-829$ & 1 & 0.1 & 0.0 & 0.6 \\
\hline -Dislocation & 830-839 & 1 & 0.1 & 0.0 & 0.9 \\
\hline -Sprains \& Strains - Other & $840-845,848$ & 5 & 0.4 & 0.1 & 0.9 \\
\hline -Open Wound - Head, Neck, Trunk & $870-879$ & 1 & 0.1 & 0.0 & 0.6 \\
\hline -Open Wound - Upper Limb & 880-887 & 3 & 1.0 & 0.2 & 5.4 \\
\hline -Open Wound - Lower Limb & 890-897 & 2 & 0.9 & 0.2 & 5.5 \\
\hline -Superficial Injury & 910-919 & 1 & 0.1 & 0.0 & 0.6 \\
\hline -Contusion & $920-924$ & 1 & 0.1 & 0.0 & $\overrightarrow{0.6}$ \\
\hline -Foreign Body Entering Orifice & 930-939 & 1 & 0.1 & 0.0 & 0.4 \\
\hline Total & & 24 & 4.4 & 2.3 & 8.5 \\
\hline
\end{tabular}

* Only those diagnostic categories with at least one occurrence appear in this table.

**Standardized to age distribution of 2000 U.S. population. 


\section{Savannah River Site 2008}

OSHA Data

Appendix W. Age-Adjusted OSHA Illness and Injury Rates by Diagnostic Category*

Part 2. Women

\begin{tabular}{|c|c|c|c|c|c|}
\hline & & $\begin{array}{l}\text { Number of } \\
\text { Diagnoses }\end{array}$ & $\begin{array}{c}\text { Age-Adjusted } \\
\text { Rate per } 1,000 * *\end{array}$ & $\begin{array}{c}\text { Lower } 95 \% \\
\text { Confidence } \\
\text { Limit per } 1,000\end{array}$ & $\begin{array}{c}\text { Upper } 95 \% \\
\text { Confidence } \\
\text { Limit per 1,000 }\end{array}$ \\
\hline Diagnostic Category & ICD-9-CM Code & \multirow[b]{2}{*}{1} & \multirow[b]{2}{*}{0.2} & \multirow[b]{2}{*}{0.0} & \multirow[b]{2}{*}{1.4} \\
\hline ENDOCRINE/METABOLIC/IMMUNITY & $240-279$ & & & & \\
\hline -Other Metabolic \& Immunity Disorders & $270-279$ & 1 & 0.2 & 0.0 & 1.4 \\
\hline $\begin{array}{l}\text { MUSCULOSKELETAL \& CONNECTIVE } \\
\text { TISSUE }\end{array}$ & 710-739 & 4 & 2.6 & 0.9 & 7.5 \\
\hline -Arthropathies & $710-719$ & 2 & 1.6 & 0.4 & 6.4 \\
\hline -Dorsopathies & $720-724$ & 1 & 0.2 & 0.0 & 1.4 \\
\hline -Rheumatism, Excluding Back & 725-729 & 1 & 0.8 & 0.1 & 5.7 \\
\hline $\begin{array}{l}\text { SYMPTOMS, SIGNS, \& ILL-DEFINED } \\
\text { CONDITIONS }\end{array}$ & $780-799$ & 1 & 0.8 & 0.1 & 5.7 \\
\hline -Symptoms & 780-789 & 1 & 0.8 & 0.1 & 5.7 \\
\hline INJURY \& POISONING & $800-999$ & 17 & 5.6 & 2.7 & 11.6 \\
\hline -Fracture - Upper Limb & $810-819$ & 1 & 0.2 & 0.0 & 1.7 \\
\hline -Fracture - Lower Limb & $820-829$ & 2 & 0.4 & 0.1 & 1.6 \\
\hline -Sprains \& Strains - Back & 846-847 & 2 & 1.0 & 0.2 & 5.0 \\
\hline -Sprains \& Strains - Other & $840-845,848$ & 1 & 0.2 & 0.0 & 1.4 \\
\hline -Open Wound - Upper Limb & 880-887 & 1 & 1.7 & 0.2 & 12.4 \\
\hline -Open Wound - Lower Limb & 890-897 & 1 & 0.2 & 0.0 & 1.4 \\
\hline -Superficial Injury & 910-919 & 2 & 0.4 & 0.1 & 1.6 \\
\hline -Contusion & $920-924$ & 4 & 0.8 & 0.3 & 2.1 \\
\hline -Foreign Body Entering Orifice & 930-939 & 1 & 0.2 & 0.0 & 1.7 \\
\hline -Toxic Effects - Non-medicinal & $980-989$ & 2 & 0.4 & 0.1 & 1.7 \\
\hline Total & & 23 & 9.2 & 5.2 & 16.1 \\
\hline
\end{tabular}

* Only those diagnostic categories with at least one occurrence appear in this table.

**Standardized to age distribution of 2000 U.S. population. 


\section{Savannah River Site 2008}

\section{OSHA Data}

Appendix W. Age-Adjusted OSHA Illness and Injury Rates by Diagnostic Category*

Part 3. Men and Women

\begin{tabular}{|c|c|c|c|c|c|}
\hline & & $\begin{array}{l}\text { Number of } \\
\text { Diagnoses }\end{array}$ & $\begin{array}{c}\text { Age-Adjusted } \\
\text { Rate per } 1,000 * *\end{array}$ & $\begin{array}{c}\text { Lower } 95 \% \\
\text { Confidence } \\
\text { Limit per } 1,000\end{array}$ & $\begin{array}{c}\text { Upper } 95 \% \\
\text { Confidence } \\
\text { Limit per 1,000 }\end{array}$ \\
\hline Diagnostic Category & ICD-9-CM Code & \multirow[b]{2}{*}{1} & \multirow[b]{2}{*}{0.0} & \multirow[b]{2}{*}{0.0} & \multirow[b]{2}{*}{0.3} \\
\hline ENDOCRINE/METABOLIC/IMMUNITY & 240-279 & & & & \\
\hline -Other Metabolic \& Immunity Disorders & 270-279 & 1 & 0.0 & 0.0 & 0.3 \\
\hline RESPIRATORY SYSTEM & $460-519$ & 2 & 0.2 & 0.0 & 0.7 \\
\hline -Lung Dis from External Agents & $500-508$ & 2 & 0.2 & 0.0 & 0.7 \\
\hline $\begin{array}{l}\text { MUSCULOSKELETAL \& CONNECTIVE } \\
\text { TISSUE }\end{array}$ & 710-739 & 9 & 1.4 & 0.6 & 3.4 \\
\hline -Arthropathies & $710-719$ & 3 & 0.3 & 0.1 & 0.9 \\
\hline -Dorsopathies & $720-724$ & 2 & 0.5 & 0.1 & 2.7 \\
\hline -Rheumatism, Excluding Back & 725-729 & 4 & 0.6 & 0.2 & 2.4 \\
\hline $\begin{array}{l}\text { SYMPTOMS, SIGNS, \& ILL-DEFINED } \\
\text { CONDITIONS }\end{array}$ & 780-799 & 1 & 0.1 & 0.0 & 0.8 \\
\hline -Symptoms & $780-789$ & 1 & 0.1 & 0.0 & 0.8 \\
\hline INJURY \& POISONING & $800-999$ & 34 & 3.4 & 1.9 & 6.1 \\
\hline -Fracture - Upper Limb & 810-819 & 2 & 0.1 & 0.0 & 0.5 \\
\hline -Fracture - Lower Limb & $820-829$ & 3 & 0.1 & 0.0 & 0.5 \\
\hline -Dislocation & 830-839 & 1 & 0.1 & 0.0 & 0.8 \\
\hline -Sprains \& Strains - Back & 846-847 & 2 & 0.2 & 0.0 & 0.7 \\
\hline -Sprains \& Strains - Other & $840-845,848$ & 6 & 0.3 & 0.1 & 0.7 \\
\hline -Open Wound - Head, Neck, Trunk & 870-879 & 1 & 0.1 & 0.0 & 0.4 \\
\hline -Open Wound - Upper Limb & 880-887 & 4 & 1.2 & 0.3 & 4.4 \\
\hline -Open Wound - Lower Limb & $890-897$ & 3 & 0.7 & 0.1 & 3.5 \\
\hline -Superficial Injury & 910-919 & 3 & 0.1 & 0.0 & 0.5 \\
\hline -Contusion & 920-924 & 5 & 0.2 & 0.1 & 0.6 \\
\hline -Foreign Body Entering Orifice & 930-939 & 2 & 0.1 & 0.0 & 0.4 \\
\hline -Toxic Effects - Non-medicinal & $980-989$ & 2 & 0.1 & 0.0 & 0.4 \\
\hline Total & & 47 & 5.1 & 3.2 & 8.1 \\
\hline
\end{tabular}

* Only those diagnostic categories with at least one occurrence appear in this table.

**Standardized to age distribution of 2000 U.S. population. 